\title{
Novel Techniques for Tissue Imaging and Characterization Using Biomedical Ultrasound
}

\author{
Ahmed M.Ehab Mahmoud \\ West Virginia University
}

Follow this and additional works at: https://researchrepository.wvu.edu/etd

\section{Recommended Citation}

Mahmoud, Ahmed M.Ehab, "Novel Techniques for Tissue Imaging and Characterization Using Biomedical Ultrasound" (2009). Graduate Theses, Dissertations, and Problem Reports. 4495.

https://researchrepository.wvu.edu/etd/4495

This Dissertation is protected by copyright and/or related rights. It has been brought to you by the The Research Repository @ WVU with permission from the rights-holder(s). You are free to use this Dissertation in any way that is permitted by the copyright and related rights legislation that applies to your use. For other uses you must obtain permission from the rights-holder(s) directly, unless additional rights are indicated by a Creative Commons license in the record and/ or on the work itself. This Dissertation has been accepted for inclusion in WVU Graduate Theses, Dissertations, and Problem Reports collection by an authorized administrator of The Research Repository @ WVU.

For more information, please contact researchrepository@mail.wvu.edu. 


\title{
Novel Techniques for Tissue Imaging and Characterization Using Biomedical Ultrasound
}

\author{
Ahmed M. Ehab Mahmoud \\ Dissertation \\ Submitted to the College of Engineering and Mineral Resources at West Virginia \\ University in partial fulfillment of the requirements for the degree of
}

\author{
Doctor of Philosophy \\ In \\ Mechanical Engineering
}

\author{
Osama Mukdadi, Ph.D., Committee Chair \\ Hany Ammar, Ph.D., Committee Member \\ Larry Banta, Ph.D., Committee Member \\ Tim McGraw, Ph.D., Committee Member \\ Victor Mucino, Ph.D., Committee Member
}

Department of Mechanical and Aerospace Engineering

Morgantown, West Virginia

2009

Keywords: Guided Waves, High-Frequency Ultrasound, Tissue Imaging, NonInvasive Measurements

\section{Copyright 2009 Ahmed M. Ehab Mahmoud}




\begin{abstract}
Novel Techniques for Tissue Imaging and Characterization Using Biomedical Ultrasound
\end{abstract}

Ahmed M. Ehab Mahmoud

The use of ultrasound technology in the biomedical field has been widely increased in recent decades. Ultrasound modalities are considered more safe and cost effective than others that use ionizing radiation. Moreover, the use of high-frequency ultrasound provides means of high-resolution and precise tissue assessment. Consequently, ultrasound elastic waves have been widely used to develop non-invasive techniques for tissue assessment. In this work, ultrasound waves have been used to develop non-invasive techniques for tissue imaging and characterization in three different applications.

Currently, there is a lack of imaging modalities to accurately predict minute structures and defects in the jawbone. In particular, the inability of 2D radiographic images to detect bony periodontal defects resulted from infection of the periodontium. They also may carry known risks of cancer generation or may be limited in accurate diagnosis scope. Ultrasonic guided waves are sensitive to changes in microstructural properties, while high-frequency ultrasound has been used to reconstruct high-resolution images for tissue. The use of these ultrasound techniques may provide means for early diagnosis of marrow ischemic disorders via detecting focal osteoporotic marrow defect, chronic nonsuppurative osteomyelitis, and cavitations in the mandible (jawbone). The first part of this work investigates the feasibility of using guided waves and high frequency ultrasound for non-invasive human jawbone assessment. The experimental design and the signal/image processing procedures for each technique are developed, and multiple in vitro studies are carried out using dentate and non-dentate mandibles. Results from both the ultrasonic guided waves analysis and the high frequency 3D echodentographic imaging suggest that these techniques show great potential in providing non-invasive methods to characterize the jawbone and detect periodontal diseases at earlier stages.

The second part of this work describes indirect technique for characterization via reconstructing high-resolution microscopic images. The availability of well-defined genetic strains and the ability to create transgenic and knockout mice makes mouse models extremely significant tools in different kinds of research. For example, noninvasive measurement of cardiovascular function in mouse hearts has become a valuable need when studying the development or treatment of various diseases. This work describes the development and testing of a single-element ultrasound imaging system that can reconstruct high-resolution brightness mode (B-mode) images for mouse hearts and blood vessels that can be used for quantitative measurements in vitro. Signal processing algorithms are applied on the received ultrasound signals including filtering, focusing, and envelope detection prior to image reconstruction. Additionally, image enhancement techniques and speckle reduction are adopted to improve the image resolution and quality. The system performance is evaluated using both phantom and in vitro studies using isolated mouse hearts and blood vessels from APOE-KO and its wild type control. This imaging system shall provide a basis for early and accurate detection of different kinds of diseases such as atherosclerosis in mouse model.

The last part of this work is initialized by the increasing need for a non-invasive method to assess vascular wall mechanics. Endothelial dysfunction is considered a key factor in the development of atherosclerosis. Flow-mediated vasodilatation (FMD) 
measurement in brachial and other conduit arteries has become a common method to asses the endothelial function in vivo. In spite of the direct relationship that could be between the arterial wall multi-component strains and the FMD response, direct measurement of wall strain tensor due to FMD has not yet been reported in the literature. In this work, a noninvasive direct ultrasound-based strain tensor measuring (STM) technique is presented to assess changes in the mechanical parameters of the vascular wall during post-occlusion reactive hyperemia and/or FMD, including local velocities and displacements, diameter change, local strain tensor and strain rates. The STM technique utilizes sequences of B-mode ultrasound images as its input with no extra hardware requirement. The accuracy of the STM algorithm is assessed using phantom, and in vivo studies using human subjects during pre- and post-occlusion. Good correlations are found between the post-occlusion responses of diameter change and local wall strains. Results indicate the validity and versatility of the STM algorithm, and describe how parameters other than the diameter change are sensitive to reactive hyperemia following occlusion. This work suggests that parameters such as local strains and strain rates within the arterial wall are promising metrics for the assessment of endothelial function, which can then be used for accurate assessment of atherosclerosis. 


\section{Acknowledgments}

First and foremost, I offer my sincerest gratitude to God who blessed me with the knowledge and give me the strength to achieve my goals in this thesis. This work or any work would not be achieved without the care of my God, so thanks God for every thing. After that, the family comes, I would like to express my gratitude to my family that have patiently supported and encouraged me throughout my life and education, and I must dedicate this work to my mother "Omayma", and my wife "Radwa".

Throughout my Ph.D., I have been fortunate to have Dr. Osama Mukdadi as my thesis advisor. It is difficult to overstate my gratitude to him for the incurable optimism and helpful suggestions that kept my research on track. I have benefited tremendously from our honest and frank conversations about research, careers, and life in general. Also, I would like to thank my M.S. advisor, Dr. Yasser Kadah, as he taught me the bases of ultrasound research and give me his continuous support.

I am really indebted to many of our collaborators, in West Virginia University, who provided us with the resources, cooperation, and time in order to achieve the goals of this work. I am especially grateful to Dr. Richard Crout and Dr. Peter Ngan from the school of dentistry, Dr. S. Jamal Mustafa, Dr. Jefferson Frisbee, Dr. Bunyen Teng, and Phoebe Stapleton from the center for Cardiovascular and Respiratory Sciences, and Dr. Hany Ammar and Dr. Tim McGraw from the department Computer Science and Electrical Engineering.

I would like to acknowledge my friend and colleague Dr. Ayman Abaza for providing me with his support and nice friendship that helped me overcoming many difficulties. Also, I would like to thank my former colleague and team member Dr. Daniel Cortes for his help in performing some mechanical designs and the nice research time we had together.

Finally, I would like to thank my teachers within West Virginia University, for the knowledge, friendly atmosphere, and support they provided me. I am grateful also to the college secretaries, particularly, in the department of Mechanical and Aerospace Engineering, West Virginia University, for helping the department to run smoothly and for assisting me in many different ways. 


\section{TABLE OF CONTENTS}

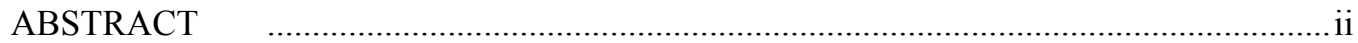

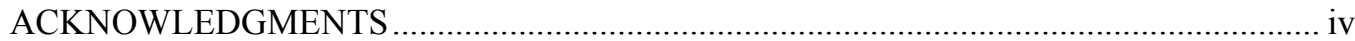

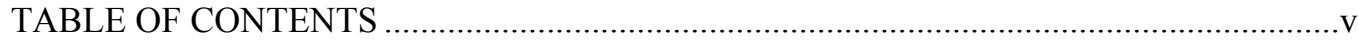

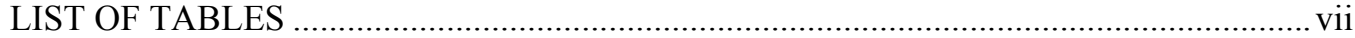

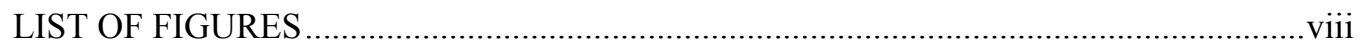

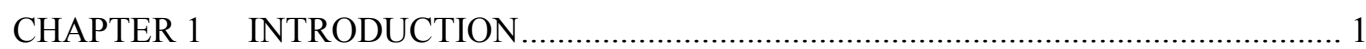

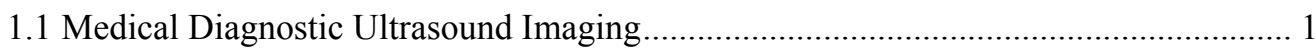

1.2 Diagnostic Ultrasound Imaging Configurations ........................................................ 2

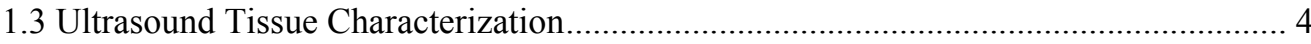

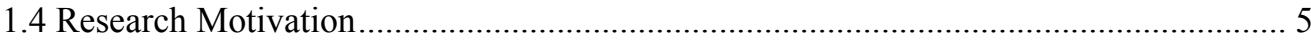

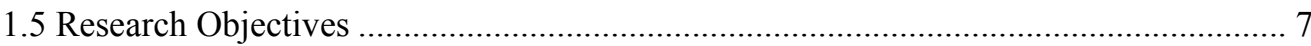

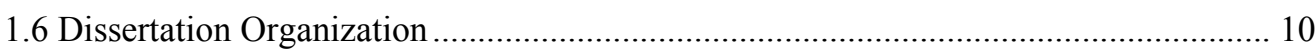

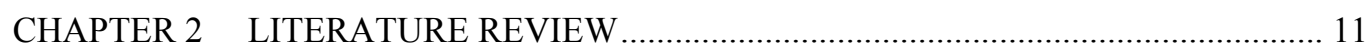

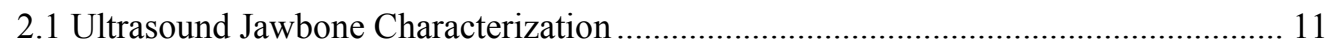

2.2 High Frequency Ultrasound Imaging for Small Animals ......................................... 13

2.3 Ultrasound Vascular Wall Tissue Characterization......................................................... 15

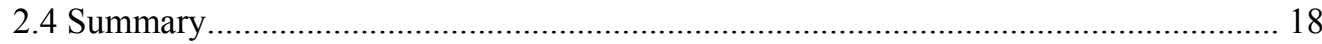

CHAPTER 3 NON-INVASIVE ASSESSMENT OF HUMAN JAWBONE USING

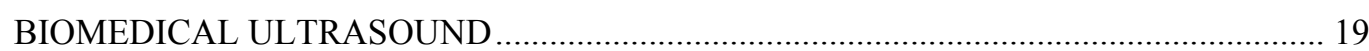

3.1 Non-Invasive Assessment of Human Jawbone Using Ultrasonic Guided Waves........ 19

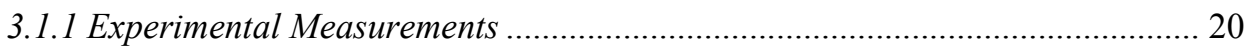

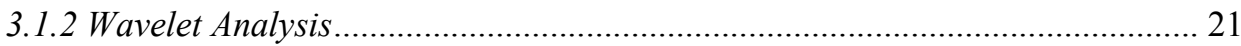

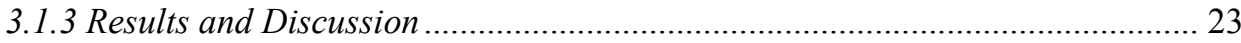

3.2 High Frequency 3D Echodentographic Imaging Modality for Early Assessment of

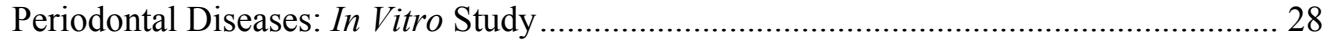

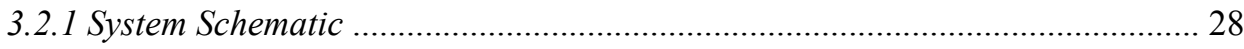

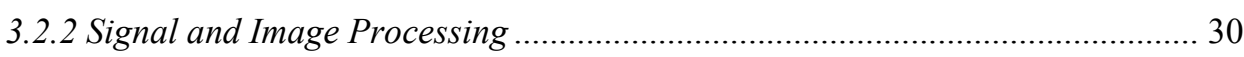

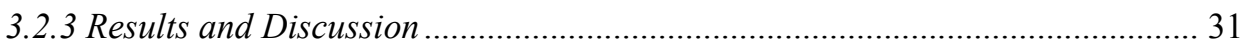

CHAPTER 4 HIGH FREQUENCY PRECISE ULTRASOUND IMAGING SYSTEM FOR

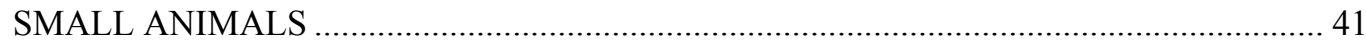

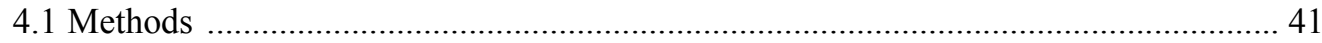

4.1.1 System Schematic ...................................................................................... 41

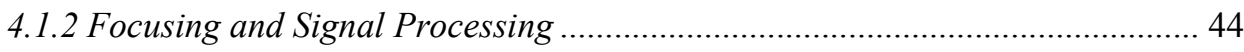

4.1.3 Image Processing and Resolution Improvement .............................................. 47

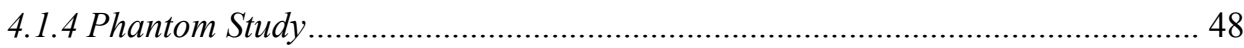


4.1.5 In Vitro Study 48

4.2 Results and Discussion 49

CHAPTER 5 IN VIVO VASCULAR WALL TISSUE CHARACTERIZATION USING A STRAIN TENSOR MEASURING (STM) TECHNIQUE FOR FLOW-MEDIATED

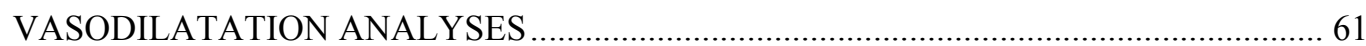

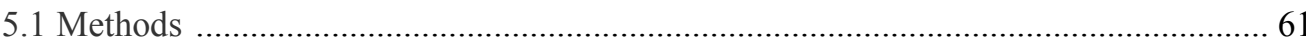

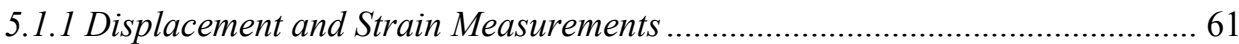

5.1.2 Displacement Measurements: In Vitro Validation Study ................................. 67

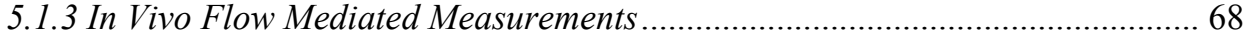

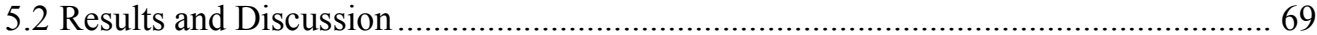

CHAPTER 6 CONCLUSION AND FUTURE WORK …................................................... 84

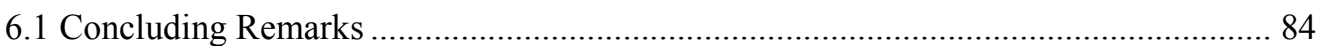

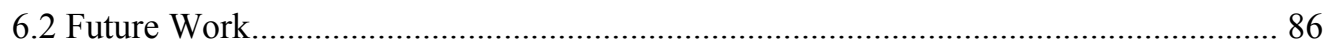

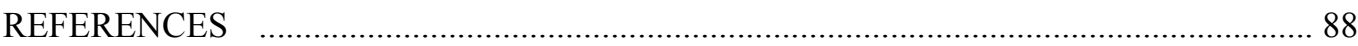




\section{LIST OF TABLES}

TABLE PAGE

1.1 General Classifications of Ultrasonic Diagnostic Instruments 3

4.1 Ultrasound Transducers Technical Description 43

Correlation $(|\mathrm{r}|)$ between the post-occlusion response of diameter

5.1 change and local wall strains (radial, longitudinal, and shear) for 5 subjects. The amplitude of correlation coefficient $|r|$ is calculated using 95\% confidence interval $(\mathrm{P}<0.05)$ 


\section{LIST OF FIGURES}

FIGURE

PAGE

3.1 Block diagram for the experimental set up

Typical RF signal received at $1 \mathrm{MHz}$ excitation frequency. a)

3.2 time-domain signal filtered by band pass filter $(0.9-1.1 \mathrm{MHz})$, and b) frequency spectrum of the raw signal

Wavelet-based experimental dispersion behavior in the high

3.3 frequency range $(0.7 \mathrm{MHz}-1.2 \mathrm{MHz})$ for the healthy human mandible VS the theoretically calculated modes arrival time at $0.9,1$, and $1.1 \mathrm{MHz}$.

Wavelet-based experimental dispersion behavior in the high

3.4 frequency range $(0.7 \mathrm{MHz}-1.2 \mathrm{MHz})$ for the defected human mandible VS the theoretically calculated modes arrival time at $0.9,1$, and $1.1 \mathrm{MHz}$

3.5 Schematic diagram showing the design and processing steps of the high-frequency ultrasound system for jawbone

Defected dentate dried cadaver mandible. a) Photographic image for the mandible with a rectangle showing the scanned region and three landmarks, b) the corresponding X-ray

3.6 radiographic images showing landmarks \# 1, 2 and 3, and (c)(e) different views for the 3D ultrasound surface image of the jawbone surface. It is clearly observed that the X-ray images have very poor information about landmarks \#2, and \#3, and limited information to perfectly describe landmark \#1

Defected dentate dried cadaver mandible. a) Photographic image for the mandible with an arrow showing a bony defect adjacent to the distal root of the first molar, b) the

3.7 corresponding X-ray radiographic image with an arrow describing the region of defect, and (c)-(e) different views for the $3 \mathrm{D}$ ultrasound surface image of the jawbone surface. It is clearly observed that the X-ray image provides no information about the bony defect described by the arrow.

Defected dentate dried cadaver mandible. a) Two Photographic images for the mandible with arrows showing two landmarks around the third molar, $\mathrm{b}$ ) the corresponding X-ray radiographic image with an arrow describing landmark \#1, and (c)-(e)

3.8 different views for the 3D ultrasound surface image of the jawbone surface. The 3D ultrasound images were able to detect different bony defects which couldn't be defined using X-ray images at landmark \#1 (sever defect), and landmark \#2 (early stage defect).

Defected dentate dried cadaver mandible with gum-tissue mimicking material. a) Photograph images for the jaw before 3.9 and after adding the tissue layer showing the three landmarks, b) an X-ray radiography image showing the same landmarks, and (c)-(e) different views for the 3D ultrasound surface image of the jawbone surface 
4.1 Block diagram of the high-frequency ultrasound system for small animal imaging. System design and processing steps are shown

4.2 Schematic of scanning on the fly from different positions

(a) Sketch for virtual-source concept. (b) Focusing geometry for the SAFT with a virtual source, where $z$ is the depth of the 4.3 synthetic focal point $p, z_{f}$ is the transducer's focal depth, $r$ is the axial distance from the transducer's focal depth to the synthetic focal depth, $r$ ' is the distance from the virtual source $f_{i}$ to the synthetic focal point $p$ [88].

B-mode images for steel wire phantom of $25 \mu \mathrm{m}$ of diameter immerged in degassed water acquired using $100 \mathrm{MHz}$ transducer. Images reconstructed using the synthetic aperture 4.4 focusing technique. The wire is placed $3 \mathrm{~mm}$ beyond the focus. Different weighting windows are used: (a) Raw Image, (b) Image reconstructed using SAFT with Hamming window, (c) cosine window, and (d) boxcar.

Axial and lateral beam plot for the $25 \mu \mathrm{m}$ steel wire using the $100 \mathrm{MHz}$ transducer. Plots show the change in the beam 4.5 spreading with different weighting windows. Boxcar, cosine, and Hamming windows are used. (a) Axial plot, and (b) Lateral plot.

Spatial resolution enhancement shown using 2D point spread function (PSF). A bird's eye view of a) raw image, b) image 4.6 after applying weighted SAFT, and c) image reconstructed after applying 2D PSF deconvolution technique, and d) axial and lateral PSF plots showing axial and lateral resolutions of 18 and $57 \mu \mathrm{m}$, respectively.

(a) Schematic of wire-phantom, (b) B-mode image of the wire phantom using our system with adaptive weighted SAFT only (c) B-mode image of a wire phantom [91], and (d). B-mode 4.7 image for the wire phantom using both adaptive SAFT and dynamic deconvolution. The use of both techniques overcomes the problem of spatial resolution degradation, shown in (c), where the lateral resolution is almost homogenous at different depths.

4.8 Photographic images describe the size in $\mathrm{mm}$ for a typical mouse heart. (a) Short axis, and (b) long axis.

Images of the long axis of 8-weeks mouse heart. (a) Image after logarithmic compression stage, and (b) image after advanced image processing stage. $R V$, right ventricle. $L V$, left ventricle. RA, right atrium. AO, Aorta. LA, Left Atrium.

Final B-mode images of the short axis of 8-weeks mouse heart.

4.10 Different slices acquired $1 \mathrm{~mm}$ apart. $\mathrm{RV}$, right ventricle. $\mathrm{LV}$, left ventricle. RA, right atrium. LA, left atrium. S, septum. Images of the long axis of 8-weeks mouse heart. Different 4.11 slices acquired $1 \mathrm{~mm}$ apart. $\mathrm{RV}$, right ventricle. $\mathrm{LV}$, left ventricle. RA, right atrium. LA, left atrium. TV, tricuspid valve. 
PA, pulmonary artery.

B-mode ultrasound images for the short axis view of isolated

4.12 mouse heart of 20-weeks APOE-HFD-Male. Different slices acquired $1 \mathrm{~mm}$ apart. RV: right ventricle. LV: left ventricle; MV: mitral valve.

B-mode ultrasound images for the long axis view of isolated mouse heart of 20-weeks APOE-HFD-Male. Different slices acquired $0.5 \mathrm{~mm}$ apart. RV: right ventricle. LV: left ventricle; LA: left atrium; MV: mitral valve; AR: aortic root.

APOE-HFD- Male-34 week-cross section. a) Image acquired using a camera on the tip high magnification microscope. b) Image acquired using our system ex vivo near the cross section in (a) before cut. RV: right ventricle.

Schematic overview of the algorithm procedures required for

5.1 measuring the arterial wall mechanical properties. Using the measured local velocity components, other parameters can be calculated by further integration or differentiation steps.

Brachial artery longitudinal view. (a) Schematic showing the 5.2 ultrasound transducer alignment relative to the field of view, and (b) B-mode ultrasound image for the brachial longitudinal view.

5.3 The Speckle tracking technique is demonstrated using a Bmode ultrasound image for one of the arterial walls.

Simple schematic for the phantom experiment showing the

5.4 radial and longitudinal directions. Pure radial, pure longitudinal and combined radial and longitudinal displacements are applied to the sponge phantom.

B-mode ultrasound images for the phantom when different

5.5 displacements are applied. The arrows indicate the directions and the relative displacement amplitudes. (a) Pure longitudinal, (b) pure radial, and (c) both longitudinal and radial.

B-mode ultrasound images for the brachial artery of healthy subject (27-year-old male). Superimposed arrows indicate the 5.6 direction and amplitude of velocity components. (a) Image acquired using the GE system, and (b) image acquired with the Sonix RP system.

Average radial velocity and diameter changes measured within a region of interest within the brachial artery of a healthy subject (26-year-old male). (a) Velocity changes in both walls, and (b) diameter change

Average radial, longitudinal, and shear strain rates and strain components changes measured within a small region $(\approx 5 \mathrm{~mm} \times$

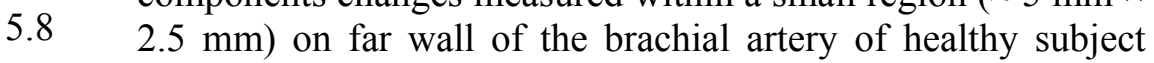
(27-year-old male). (a) Radial component, (b) longitudinal component, (c) and shear component.

5.9 Average diameter change pre- and post-occlusion (about 20 sece (a) pre-occlusion, and (b) post-occlusion. 
Average radial strain rate and strain change pre- and post5.10 occlusion. Values are measured within a small region $(\approx 5 \mathrm{~mm}$ $\times 2.5 \mathrm{~mm}$ ) on far wall of the brachial artery of healthy subject (26-year-old male). (a) Pre-occlusion, and (b) post-occlusion.

Average longitudinal strain rate and strain change pre- and post-occlusion. Values are measured within a small region $(\approx 5$

$5.11 \mathrm{~mm} \times 2.5 \mathrm{~mm}$ ) on far wall of the brachial artery of healthy subject (26-year-old male). (a) Pre-occlusion, and (b) postocclusion.

Average shear strain rate and strain change pre- and postocclusion. Values are measured within a small region $(\approx 5 \mathrm{~mm}$ $\times 2.5 \mathrm{~mm}$ ) on far wall of the brachial artery of healthy subject (26-year-old male). (a) Pre-occlusion, and (b) post-occlusion.

Frequency spectrum of the radial component of the far wall 5.13 motion of healthy subject (26-year-old male). (a) Pre-occlusion, and (b) post-occlusion. 


\section{CHAPTER 1}

\section{INTRODUCTION}

Virtually every pregnant woman, whether in western or eastern countries will have her fetus clinically evaluated with the aid of ultrasound imaging. Ultrasound is defined as acoustic waves with frequencies above those detected by human ear, from about $20 \mathrm{kHz}$ up to several hundred $\mathrm{MHz}$ [1]. Ultrasound was first used, in 1935, in England for the detection of cracks and defects in railroads, airplane, and submarine structures. After World War II fundamental ultrasound technology was available to be applied to medical field. Since then this technology has been widely used in medical application, especially in the field of medical imaging and non-invasive characterization. In this thesis, we are investigating novel ultrasound techniques for non-invasive tissue characterization and imaging in three different areas including dental imaging and tissue characterization, small animal imaging, and cardiovascular tissue characterization. In this chapter, we will discuss the basics of ultrasound medical imaging and give a brief introduction about ultrasound tissue characterization.

\subsection{Medical Diagnostic Ultrasound Imaging}

Medical instrumentation typically uses a portion of ultrasound spectrum ranging from 1 to $10 \mathrm{MHz}$ to improve both the image resolution (small wavelength) and the penetrating ability, which is inversely proportional to the wave frequency [2]. In some specialized applications in which targets are superficial and high resolution is required, very high frequencies up to $100 \mathrm{MHz}$ may be used [3]. Ultrasound imaging of the heart and vasculatures is commonly used by many clinicians to diagnose patients suffering from chest pain. In many parts of the body, suspected tumors are routinely scanned with clinical ultrasound. This widespread use is evidence of the unique advantages offered by ultrasound, which complements other important diagnostic imaging technologies such as MRI, PET, and X-rays. Compared to most of these, an ultrasound scanner is small, lightweight, inexpensive, and most importantly 
is noninvasive. This technology requires only a handheld transducer head and processing and display electronics. Ultrasound imaging is indeed non-ionizing, safe and produces few or no side effects. It also has the advantage of being able to probe the mechanical properties of human tissue directly, while other techniques must infer this indirectly [4]. Therefore, several techniques for noninvasive tissue characterization and imaging were developed using ultrasound elastic waves [2].

Medical diagnostic ultrasound uses acoustic energy that interacts with the acoustic properties of the body to produce an image from stationary and moving tissues. Ultrasound is commonly used in pulse-echo format whereby pulses of ultrasound waves, produced over a very short time, travel through various tissues and are reflected by tissue boundaries back to the transducer. Returning echoes carry the ultrasound information that is used to create the sonogram or measure blood velocities with Doppler frequency techniques. Along a given beam path, the depth of an echoproducing structure is determined by the time between the pulse-emission and the echo return, and the amplitude of the echo is encoded as a gray-scale value. In addition to 2D imaging, ultrasound provides anatomic distance and volume measurements, motion studies, blood velocity measurements and 3D imaging [5].

\subsection{Diagnostic Ultrasound Imaging Configurations}

In this section, we categorize examples of important classes of instruments used for clinical ultrasound imaging and discuss major applications in which they are used. Table 1.1 outlines commonly used classification schemes for the general configurations utilized in medical ultrasound technology. Ultrasound amplitude mode (A-mode), brightness mode (B-mode), and constant depth mode (C-mode) instruments give spatial information about the region of interest, whereas the ultrasound motion mode (M-mode) and continuous wave Doppler devices provide motion or velocity information. One of the earliest ultrasound imaging techniques used is the A-mode, which provides one-dimensional information. However, this technique is still viable for many applications including midline echoencephalography and in ophthalmological scanning. However, it has been largely supplanted by the development of the two-dimensional B-mode imaging, which provides further detailed spatial information of the scanned object. As can be seen from table 1.1, the 
category of B-scanners makes up the largest group of instruments now in use. The most sophisticated instruments, and the ones that generally provide the greatest amount of data and therefore are the most expensive, are the electronically B-mode ultrasound scanners equipped with pulsed Doppler techniques. Many recent devices are capable of operating in a combination of modes, such as an M-mode line interlaced in a real-time B-mode sector, or the combination of pulsed Doppler and Bmode instruments (duplex scanner). Such advanced techniques play a role in providing detailed information of the region of interest using multi-imaging modalities.

Table 1.1 General Classifications of Ultrasonic Diagnostic Instruments [1]

A-Mode (one-dimensional)

B-Mode (two-dimensional)

Manual scanners

Real-time scanners

Mechanical

Electronic

Linear array

Phase array

M-mode (motion)

C-mode (through-transmission)

Doppler (velocity)

Continuous-wave

Audible-output

Analog-output

Spectrum-output

Doppler imagers

CW transverse scanners

Pulsed Doppler

Combined with B-mode (duplex)

Miscellaneous 


\subsection{Ultrasound Tissue Characterization}

The development of ultrasonic tissue characterization (UTC) is the untangling of hidden patterns in radio frequency (RF) data to extract more information about tissue function and pathology than that seen in conventional images [6]. Tissue characterization was originally conceived by Dr. J. J. Wild and J. M. Reid in 1952, and was a type of remote painless ultrasound telehistology (a noninvasive way of determining the health of tissue or organ function through calculations and parameterized inferences from ultrasound data). Since then, this branch of medical ultrasound has undergone considerable development. Recent methods include specialized measurements, signal processing, statistical analysis, and parameterized imaging. Most of UTC techniques are based on RF signals either before or after signal beamforming. These methods based exclusively on processing RF data are basic spectral method, spectral features, integrated backscatter, and signal processing. A major goal in UTC is classifying and revealing the properties of the tissue through wave backscattering or other ultrasound measured parameters. Another goal is to use acquired information from RF signals to differentiate between healthy or diseased tissues. This allows many clinicians to detect pathological changes in tissues due to stimulus or medication [2]. High-frequency ultrasound is opening up new sites not detected at conventional frequencies below $15 \mathrm{MHz}$. Many clinical applications requiring the development of high-frequency ultrasound imaging including: 1) identify plaques in small blood vessels such as coronary artery using intravascular ultrasound (IVUS); 2) examining biomarkers and cellular structures; 3) imaging small animals such as mice, which are widely used as a disease model for humans; 4) diagnosing periodontal diseases and imaging tiny boney defects; 5) assessing peripheral vascular tissues and atherosclerosis in human...etc. These applications require advances in high-frequency ultrasound imaging techniques that would provide a tool for monitoring the progression of a disease, and thus could be categorized as a non-invasive tool for tissue classification. 


\subsection{Research Motivation}

This work is basically motivated by the fact that ultrasound waves combine multiple characteristics that are beneficial for different areas in the biomedical research field. In the field of tissue imaging and characterization, ultrasound based techniques are providing flexibility, clarity, safety, and cost effectiveness. They may be considered the optimum techniques that balance between these characteristics when comparing with other techniques such as X-ray, MRI, and PET. In this work, we aim to develop techniques and designs based on ultrasound that we believe they can benefit three areas in the biomedical research field. In the following paragraphs, more details about the motivation in each of the three applications are described based on the literature review presented in chapter two.

The use of ultrasound in dentistry is still an open growing area of research. Currently, there is a lack of imaging modalities to accurately predict minute structures and defects in the jawbone easily. The problem of detecting small defects in jawbones is a challenging problem. Existing methods based on X-rays are invasive and constrain the achievable image quality. They also carry known cancer risks and may be limited in an accurate diagnosis scope. The first part of this work is motivated by the lack of current imaging modalities to accurately detect tiny defects in jawbone. Ultrasonic waves are sensitive to changes in microstructural properties and thus have been widely used for noninvasive material characterization. Using these waves may provide means for early diagnosis of marrow ischemic disorders via detecting focal osteoporotic marrow defect, chronic nonsuppurative osteomyelitis, and cavitations in the mandible (jawbone). Guided waves propagating along the mandibles may exhibit dispersion behavior that depends on material properties, geometry, and embedded cavities. In this work, we present the first study in the theoretical and experimental analysis of guided wave propagation in jawbone. Also, we investigate the feasibility of utilizing high frequency ultrasound to reconstruct high resolution 3D ultrasound images from the human jawbone.

The availability of well-defined genetic strains and the ability to create transgenic and knockout mice makes mouse models extremely significant tools in different kinds of researches. For instance, apolipoprotein E knockout mice (APOE-KO) are well known to develop hyperlipidemia and atherosclerosis. Consequently, noninvasive 
measurement of cardiovascular function in mouse hearts has become a valuable need when studying the development or treatment of various diseases. Cardiovascular research based on mouse strains has motivated the development of several techniques for the assessment of cardiovascular function and morphology of living mice. Recently, several imaging techniques have emerged as promising non-invasive alternatives. Although commercial ultrasound scanners are used to image the anatomical details of human hearts, they can not be used in small animals due to the limited image resolution (1-2 mm). Most small animal imaging techniques suffer from poor spatial resolution $(>100 \mu \mathrm{m})$ in addition to the limited field of view (FOV) in some of them. The small size of mice vasculature requires a spatial resolution of 50 $\mu \mathrm{m}$ or less. In this research, we address and test techniques to enhance the spatial resolution. Although our system is capable of providing high frame-rate imaging ( 250 frame per second), the emphasis of this work is focused on improving the spatial resolution in vitro using phantoms and isolated mouse hearts and blood vessels.

Ultrasound imaging modalities has been widely used in the flow-mediated vasodilation (FMD) analysis as a noninvasive low-cost tool, which can be used to track the arterial diameter change with time. Most of the FMD measurements in the literature are based on tracing the vessel wall boundary manually. Since this process is time consuming and may introduce human errors, automatic measurement techniques have been implemented. These techniques utilize image processing algorithms to identify the edges of arterial walls, and then calculate the relative displacement change with time. Most of automatic noninvasive measurement techniques based on cardiovascular ultrasound require extra-hardware add-ons which may increase the system complexity and cost. Also, in spite of the direct relationship between the arterial wall multi-component strains and the FMD response, direct measurement of wall strain tensor due to FMD has not yet been reported in the literature. In this work, a noninvasive direct ultrasound technique to measure changes in mechanical parameters of the vascular wall is developed. These parameters include local velocities and displacements, diameter change, multi-component strain tensor and strain rates. This technique is applied during the FMD analysis to investigate how parameters other than the diameter change are sensitive to pre- and post-occlusion. 


\subsection{Research Objectives and Contributions}

As the title of this Ph.D. thesis reflects, we aim to investigate various highfrequency UTC techniques that can be applied in medical or clinical applications. First, we aim to investigate the usefulness of high-frequency ultrasound imaging for characterizing periodontal disease in humans noninvasively. Second, we aim to develop a high-frequency ultrasound imaging system that can reconstruct quantitative and high-resolution ultrasound images of small animals. Third, we aim to elucidate the usefulness of quantitative ultrasound imaging of vascular wall mechanics for diagnosing peripheral vessels and assessing endothelial functions in humans using Bmode ultrasound images in vivo.

Below, the goals for each objective are described and categorized for each application. Concerning the first part for non-invasive ultrasound jawbone characterization using ultrasound, the goals are to:

\section{A.1) Design the experimental set-up and implement the signal processing required} for analyzing guided waves propagation through jawbone

The aim is to design the experimental setup for the guided wave experiment using contact ultrasound transducers. Multiple experiments are conducted, and signal processing algorithms are developed for analyzing ultrasound signals using Wavelet techniques.

\section{A.2) Develop a high-frequency echodentographic imaging system to provide high resolution images for early diagnosis of periodontal diseases}

The aim is to design the experimental setup and the signal and image processing required for investigating the feasibility of utilizing high-frequency ultrasound for reconstructing high-resolution images of human mandibles. The system employs high frequency single-element ultrasound focused transducers $(>15 \mathrm{MHz})$ for scanning.

\section{A.3) Perform in vitro studies using normal and defected dentate and non-dentate dried mandibles to assess the system efficacy and accuracy}


The aim is to perform different in vitro studies using normal and defected dentate mandibles to assess the efficacy of the techniques mentioned above via comparing the experimental results with the theoretical results or with the jawbone anatomy.

In the second part for the development of the high frequency ultrasound imaging system for small animals, the aims are:

\section{B. 1) Develop and utilize a custom ultrasound biomicroscopy system that provides} high resolution images to accurately image tissues with microscopic resolution The aim is to design a custom ultrasound system that uses high-frequency transducers (30-120 MHz) and can provide ultrahigh spatial resolution down to $18 \mu \mathrm{m}$. The highspeed data acquisition is synchronized with the transducer movement during the collection of ultrasound signals "on-the-fly scanning".

B. 2) Implement the signal and image processing algorithms required for the reconstruction of high quality $B$-mode images from the Radio Frequency $(R F)$ data measured by ultrasound

The aim is to reconstruct high-resolution B-mode images of mouse hearts using advanced signal and image processing techniques which are implemented. Signal processing techniques include filtering and time gain adjustment, focusing, while image processing includes image enhancement and focusing techniques.

\section{B. 3) Test the proposed system using standard wire phantoms, and isolated heart samples}

The aim is to perform comprehensive validation experiments to test the usefulness of using the proposed high-frequency ultrasound imaging system:

\section{a. In vitro wire phantom}

Several experiments are performed using wire phantoms of diameters down to 25 microns. This size range is comparable to the diameter of mouse heart vasculatures, and could be used to determine the resolution experimentally. Images of the wire phantoms are reconstructed and compared with the actual geometries, and with results of other groups. 


\section{b. Ex vivo isolated mice hearts and blood vessels}

Ex vivo experiments are performed using isolated mouse hearts and blood vessels. Normal hearts and hearts with atherosclerosis will be imaged. In addition, images for the isolated samples are compared with the heart histology and images obtained using dissecting microscope.

In the last part of this work concerning the in vivo vascular wall tissue characterization application, our goals are:

\section{1) Develop a noninvasive direct ultrasound technique to measure changes in mechanical parameters of the vascular wall}

The aim is to develop a noninvasive direct ultrasound technique to measure changes in mechanical parameters of the vascular wall. These parameters include local velocities, displacements, diameter change, and local strain tensor and strain rates. The technique utilizes sequences of B-mode ultrasound images as its input with no extra hardware requirement.

\section{2) Test the proposed algorithm to measure different kinds of displacements using phantom studies}

The aim is to perform phantom studies to test the ability of the algorithm to measure small displacements in the radial, longitudinal, and both directions. A material of sponge with comparable speckle distribution and irregular boundaries is used to mimic the vascular wall tissues.

\section{3) Perform several in vivo studies to test the algorithm efficacy in measuring the vascular parameters using images from different ultrasound machines}

To assure the flexibility of this algorithm, we aim to use two different ultrasound machines to acquire the ultrasound data. All the parameters are measured using images from both the ultrasound machines using different in vivo studies.

C. 4) Apply the proposed technique on several in vivo studies during the FMD analysis to test the algorithm efficacy in measuring the vascular response 
Different in vivo experiments for human subjects are performed on FMD data. This technique is applied during the FMD analysis to investigate how parameters other than the diameter change are sensitive to pre- and post-occlusion.

The aforementioned aims illustrated for different biomedical ultrasound applications are the contributions achieved in this dissertation. These contributions are categorized and described according to each application as will be described in the next section.

\subsection{Dissertation Organization}

In the following chapters, the details of how these goals have been achieved will be explained in detail. The rest of this dissertation is organized as follows: Chapter 2 provides additional background and description of existing techniques for each application. The next three chapters discuss the design and development of each technique. Chapter 3 describes how goals $A .1, A .2$, and $A .3$ have been achieved, while the details of accomplishing goals B.1,B.2, and B.3 are shown in chapter 4 . Then, chapter 5 provides all the information regarding how the rest of the goals C.1, C.2, C.3, and C.4 are achieved. Finally, Chapter 6 concludes this thesis and provides a description of the challenging points for future work. This work has been published in the IEEE Ultrasonics Symposium, ASME Summer Bioengineering Conference, ASME IMECE, SPIE Medial Imaging, and the IEEE Transactions on Ultrasonics, Ferroelectrics, and Frequency Control (TUFFC). 


\section{CHAPTER 2}

\section{LITERATURE REVIEW}

In fact, the field of tissue characterization includes various techniques in different applications. Since this work is associated with three different areas within the tissue characterization field, more details and background about these areas are needed before introducing our work. This chapter reviews each of these areas, and presents several techniques and methods that have been used. Moreover, recent developments for tissue characterization will be addressed.

\subsection{Ultrasound Jawbone Characterization}

Noninvasive techniques for jawbone characterization are motivated by clinical need for early diagnosis of oral osteoporosis, periodontal defects, and cavitations. Osteoporosis is usually diagnosed by low bone mineralization and micro-architectural deterioration of bone tissue [7]. The diagnostic threshold identifies approximately $15-20 \%$ of postmenopausal women as having osteoporosis. Periodontal (gum) diseases are serious infections that can lead to tooth loss. Chronic periodontitis is the most common form of periodontitis resulting in inflammation within the supporting tissues of the teeth. Most adults have a mild form of periodontal disease, and over 20 percent of elder Americans suffer severe periodontal disease [8]. Cavitations pathology was described by Black [9] as: bone necrosis, resulting in persistently hollowed-out areas at the sites of old teeth extractions. Recent studies [10] established a relation between different jawbone diseases and infection from one side and chronic diseases as rheumatic diseases and heart problems from the other side. Thus, the assessment of jawbone mechanical condition is a clinically important issue.

Ultrasound imaging and evaluation technologies are very safe modalities compared to those involve ionizing radiation, such as X-ray and nuclear medicine. However, ultrasound use in dental applications is still very limited and requires further investigations. Different ultrasound techniques have been used to measure tooth layers thickness $[11,12]$, and to detect early stages of teeth cracks [13]. Lynch 
and Hinders [14] described the development and testing of an ultrasonographic periodontal probe designed to replace manual probing for measuring of periodontal attachment loss. Tsiolis et al. [15] investigated the ability of using high-frequency ultrasound imaging for periodontal assessment. Ultrasound real-time imaging was used for detecting mandibular lesions and measuring bone thickness. Cotti et al. [16] described the use of a real-time ultrasound imaging technique (echography) to study periradicular lesions (around the root). Uchida et al. [17] measured the thickness of the masticatory mucosa, using $20 \mathrm{MHz}$ B-Mode ultrasonic diagnostic equipment. Through-transmission alveolar ultrasonography was used in the detection of cavitations [18]. Through-transmission imaging of the cancellous portion of the jawbones, allow the assessment of the jawbone density and quality [19].

Ultrasonic guided waves are widely used in nondestructive testing for the assessment of plates, tubes and more complex composite structures [20]. Mechanical properties of anisotropic layered plates can be obtained by means of reconstruction of the dispersion spectrum from experimental measurements [21]. Furthermore, delaminations, cracks, and other defects can be detected using axial transmission or backscattering techniques, which may reveal changes in characteristic guided wave dispersion [22-24]. Dispersion curves of guided wave propagation in composite cylinders have been widely analyzed for gas and oil pipeline industry [25]. Additionally, guided waves are widely used for nondestructive characterization of layered finite-width plates used for semi-conductor materials [26], and for health monitoring of structures with arbitrary cross-sections used for aerospace structures and railroads $[27,28]$.

Guided waves have recently been used for ultrasound assessment of bone condition since they are sensitive to both mechanical and geometrical properties of the propagation media. Njeh et al. [29] presented a review of the role of ultrasound in the assessment of osteoporosis. Bossy et al. [30] proved potential sensitivity of axial transmission ultrasound velocity to changes in cortical bone status under different pathological conditions or treatments. Bossy et al. [31] proved by experimental study that specific emitters and receivers arrangement can eliminate the effect of different soft tissue layer thickness, and hence they accurately estimated the wave velocity along the bone layer. Raum et al. [32] used ultrasonic wave propagation in cortical bone to measure these tissue anisotropic elastic properties, porosity and the cortical 
geometry (e.g., thickness). In recent studies, Moilanen et al. [33] and Nicholson et al. [34] compared the performance of low frequency ultrasonic guided wave measurements with well established ultrasound and bone density measurements in terms of their ability to characterize the tibia bone. Lee and Yoon [35] used leaky Lamb waves to investigate the effect of cortical thickness variation. Lefebvre et al. [36] evaluated other bone mechanical properties like Young's modulus and cortical bone thickness. In this work, we present the first experimental study for guided wave propagation in jawbone and its ability to assess human mandibles. Also, we describe the development and testing of a novel high-frequency echodentographic imaging system that can be used for the early assessment of jawbone.

\subsection{High Frequency Ultrasound Imaging for Small Animals}

Genetically modified mice provide a powerful tool for understanding the molecular mechanisms and pathogenesis of human cardiovascular diseases like human atherosclerosis [37]. Numerous mouse strains are available today with phenotypes relevant to human cardiovascular diseases [37,38]. While a wide range of mouse strains has been developed and many have become very widely used in experimental studies, normal mice are short-lived and characteristically resistant to cardiovascular disease (CVD). A number of genetic mouse models based on either spontaneous mutation or genetic manipulation have been developed and used in studies of CVD [37]. These include transgenic models with deletion ("knockout") of apoB and apoE, or the LDLR [39, 40]. Some of the more widely used strains are knockout mice, $\mathrm{db} / \mathrm{db}$ mouse [41], and ob/ob mouse [42]. Moreover, knockout mice have several models like the ApoE ${ }^{-}$mouse, LDLR mouse $\%$ mouse, and the SR-BI $\mathrm{KO}$ mouse. The mouse model used in this project is the $\mathrm{ApoE}^{-/}$("knockout") mouse, which is the most widely used mouse model [43]. These mice have total plasma cholesterol concentrations of $11 \mathrm{mM}$, compared to $2 \mathrm{mM}$ for the parent C57BL/6 mouse [40]. They also exhibit advanced intimal lesions that are largely confined to the aortic root area [37]. The development of more widely distributed atherosclerosis, similar to that seen in humans, requires a high-cholesterol diet with the induction of plasma cholesterol concentrations approaching $30 \mathrm{mM}[39,44]$. Similarly, the C57BL/6 mouse develops some atherosclerosis, again in the aortic root, when fed a 
high-fat or a high-cholesterol diet [45]. Schreyer et al. [45] suggested that these mice were models of "diabetes-accelerated atherosclerosis" despite the absence of substantive insulin resistance/hyperinsulinemia, much less of overt diabetes. Overall, the $\mathrm{ApoE}^{-/}$model can be viewed as an analogue of untreated severely dyslipidemiac humans.

The common use of these novel mouse strains has prompted the development of techniques for assessing the cardiovascular function and morphology of living mice [46]. Usually, coronary artery morphology is studied using invasive techniques such as angiography. Recently, several imaging techniques have been emerging as promising non-invasive alternatives. Examples of such techniques are electron-beam computed tomography [47], magnetic resonance imaging [48], positron emission tomography (PET) [48], optical coherent tomography [50], and ultrasound biomicroscopy [51]. However, in mice, which have extremely small coronary arteries and high heart rates (HR), the coronary circulation constitutes a great challenge for these available imaging techniques. The above mentioned techniques suffer from either insufficient spatial resolution and/or poor temporal resolution. The small size of mice vasculature and the rapid heart rate (400-800 beats/minute) [52] require a high frame-rate imaging system $(>100 \mathrm{fps}$ or frames per second and a spatial resolution of $20 \mu \mathrm{m}$ or less. Image alignment is more complicated for the heart than for other organs due to the rapid and flexible motion of the myocardium, and it is a critical problem in several imaging modalities. In general, ECG is used to minimize imaging artifacts caused by cardiac motion [53]. Using high-speed high-resolution ultrasound system will allow real-time imaging with a temporal resolution at least of $250 \mathrm{fps}$ and spatial resolution less than $20 \mu \mathrm{m}$.

Turnbull et al. [54] was the first to report the use of high-frequency ultrasound to observe mutant phenotyping in the mouse embryo. Further investigations were conducted on mice by Foster et al. $[55,56]$ with an improved prototype scanner. Mouse embryonic heart development also was observed by Srinivasan et al. [57] using a noninvasive $40 \mathrm{MHz}$ UBM system built to image through the uterus. In addition to small animal applications, high-frequency ultrasound imaging also has proven to be a valuable tool in clinical imaging of the eye, skin, and vasculature $[55$, 58]. All UBM studies are currently carried out exclusively by mechanically scanning a single-element transducer. Currently, a commercial high-frequency UBM system 
for small animal imaging is available (Vevo 770, Visualsonics Inc., Toronto, ON, Canada). It can achieve a frame rate up to $240 \mathrm{fps}$ by limiting the lateral view to 1 $\mathrm{mm}$. However, this system uses ECG gating technique, where multiple echoes are collected at each point during the scan. It is analogous to the ECG gating cardiac MRI imaging technique where several ECG cycles are required to reconstruct a $2 \mathrm{D}$ image of a single ECG cycle. This limitation may not be appropriate to many echocardiographic imaging that requires real-time monitoring of cardiac response, for example, flow-mediated vasodilation analysis. Moreover, most of the technical details of that system are not revealed.

Array-based imaging systems allow electronic dynamic focusing, aperture apodization, and electronic beam steering for improved spatial resolution and frame rate. Therefore, high-frequency linear array systems would be the best solution for cardiac imaging of mice. Unfortunately, high performance linear phased arrays require a pitch, which is defined as the distance between the centers of two adjacent elements, to be no greater than half of the ultrasound wavelength to avoid grating lobes. The ultrasound wavelength is approximately $30 \mu \mathrm{m}$ at $50 \mathrm{MHz}$. Therefore, the fabrication of linear-phased array transducers at this frequency can be extremely challenging. Linear-sequenced arrays operating at 30 and $40 \mathrm{MHz}$ recently have been developed [59,60], but to further increase the frequency is not a trivial matter. Although both analog and digital beamformers are being developed [61], it may take a few years for array systems to reach a stage at which their image quality rivals that obtainable with a UBM. Moreover, the cost of array systems may be prohibitive because of their complexity.

The aim of this work is to design, test, and utilize a custom ultrasound system available in our laboratory that uses high frequency transducers and can provide ultrahigh spatial resolution down to $15 \mu \mathrm{m}$. This high frequency $(30-120 \mathrm{MHz})$ ultrasound imaging system can reconstruct high-resolution B-mode of small animal tissues.

\subsection{Ultrasound Vascular Wall Tissue Characterization}

Cardiovascular disease refers to the class of diseases that involve the heart or blood vessels (arteries and veins). While the term technically refers to any disease 
that affects the cardiovascular system, it is usually used to refer to those related to atherosclerosis (arterial disease). According to the statistics conducted by the American Heart Association Statistics Committee and Stroke Statistics Subcommittee and published in 2007, an estimated 79,400,000 American adults (1 in 3) have 1 or more types of cardiovascular diseases [62]. Atherosclerosis has become one of the major contributing factors to cardiovascular disease, and chronic endothelial dysfunction has been well established as a key factor in the development of atherosclerosis [63]. Consequently, early diagnosis for endothelial dysfunction has become an important need for prevention. Wada et al. [64] reported that atherosclerosis causes morphological and mechanical changes in arterial walls that alter their physical properties.

Morphologically, the arterial wall consists of three concentric layers called intima, media, and adventitia [65]. In normal vascular arteries, the tunica intima consists of mono-layered endothelial cells, while the media is composed mainly of smooth muscle cells. The adventitia is a layer of investing connective tissue. The media is responsible for modulation of vessel compliance, and the intima forms the interface between the artery wall and the blood stream. Therefore, endothelial cells mainly adjust the distensibility of blood vessels and maintain vascular homeostasis. Various techniques have been developed for the diagnosis and early detection of arterial diseases. Hayashi et al. [66] have used the relationship between transmural pressure and wall diameter to express the stiffness of a vascular wall. In a pilot study, Ramnarine et al. [67] have demonstrated the ability of ultrasound tissue Doppler imaging (TDI) of arterial wall motion (AWM) to differentiate between the dynamic behaviors of normal and diseased carotid arteries. They found some limitations when using TDI for simple quantitative AWM measures due to the high variability. Brands et al. and Hoeks et al. [68, 69] have described an ultrasonic system consists of three parts to measure the wall shear strain rate noninvasively. They estimated the shear rate distribution as a function of the radial derivative of the velocity profile at each time instant. In 1999, they developed an integrated ultrasound based system for noninvasive assessment of vessel wall and hemodynamic properties of large arteries [70]. Their system, ART-lab, can be connected to any echo-system providing an analogue non-compressed wideband RF signals, and then it extracts the shear rate and velocity profile using these signals. Although this system provides means of on-line 
monitoring for different parameters, it may not be compatible to several ultrasound machines that not providing access to raw RF signals. Bambi et al. [71] have described an integrated ultrasound system capable of detecting both the velocity profile and the wall movements in human arteries. It basically consists of a PC add-on board including a single high-speed digital signal processor. Two years later, they developed a technique for noninvasive real-time simultaneous assessment of both blood velocity profile and wall displacements in human arteries [72]. The technique is based on the use of two ultrasound beams, one set at optimal angle for wall motion measurements and the other for blood velocity profile measurements.

Flow-mediated vasodilation (FMD) measurement in brachial and other conduit arteries has become a common method to asses the endothelial function in vivo [73]. The FMD approach involves a short period of occlusion for the upper or lower arm that evokes dilation of the distal resistance vasculature with the forearm. On release of occlusion, there is a substantial but transient elevation in brachial artery flow velocity providing an increased shear stress stimulus and resulting in FMD. Fluid shear-stress increases due to the blood flow increase, thus stimulating endothelial cells production and release of nitric oxide, a potent endogenous vasodilator. The mechanical behavior of the arterial wall during vasodilation is considered an indication for endothelial health as mentioned before. In FMD measurement, the endothelium-dependent variation in arterial diameter in response to reactive ischemia-induced hyperemia is measured by comparing the luminal diameter of the brachial artery before and after the ischemia of the forearm induced by pressurizing a cuff [74].

Ultrasound imaging modalities have been widely used in the FMD analysis as noninvasive low-cost tools, which can be used to track the arterial diameter change with time. Most of the FMD measurements in the literature are based on tracing the vessel wall boundary manually. Since this process is time consuming and may introduce human errors, automatic measurement techniques have been implemented $[74,75]$. These techniques utilize image processing algorithms to identify the edges of arterial walls, and then calculate the relative displacement changes with time. Kaneko et al. [76] have developed a method for measuring the change in the thickness and the elasticity of the brachial artery during a cardiac cycle using the phased tracking method for the evaluation of the mechanical property of only the intima-media region. In his study, the initial positions of echoes from the lumen-intima and media- 
adventitia boundaries are determined using complex template matching to accurately estimate the minute change in the thickness and the elasticity of the brachial and radial arteries. This method was applied to the measurement of conventional FMD, the change in intima-media thickness, and the elasticity of the radial artery using highfrequency ultrasound $(10 \mathrm{MHz})$.

In spite of the direct relationship between the arterial wall local strains and FMD response, direct measurement of the wall strain tensor due to FMD has not yet been reported in the literature. In this work, a noninvasive measurement technique for arterial wall local displacements and strain tensor during FMD analysis is described. This technique extracts the mechanical measures from sequences of B-mode ultrasound images for the longitudinal section of the vessel acquired using high frequency ultrasound machines.

\subsection{Summary}

In this chapter, the role of biomedical ultrasound as a diagnostic and measurement tool in three different areas was described. Several techniques for tissue characterization and imaging were also explained. These techniques are divided into two general approaches, the RF signal analysis approach and the image analysis approach. In the first approach, the RF signals received from the target tissue are subjected to signal analysis to extract useful information about the tissue. While in the second approach, image processing and analysis techniques are applied to extract measurements and properties from ultrasound images of the target tissues. In this work, these two approaches have been utilized. In the next two chapters, chapter 3 and 4 about human jawbone and small animal imaging and tissue characterization, both approaches are integrated. That is because the processing starts with acquiring raw RF signals followed by several signal and/or image processing procedures, then ends with displaying contours/images. While in the rest of this work, chapter 5, the second approach is only used to characterize the human brachial artery, since the processing begins from the B-mode ultrasound images. 


\section{CHAPTER 3}

\section{NON-INVASIVE ASSESSMENT OF HUMAN JAWBONE USING BIOMEDICAL ULTRASOUND}

In this chapter, two ultrasound methods developed to assess jawbone are presented. The first technique describes how changes in ultrasonic guided wave propagation in jawbone are used for noninvasive assessment. The experimental set up and design procedures for this technique are explained. Also, the signal processing algorithm based on wavelet analysis is described. Then, the experimental results are shown and compared with the theoretical analysis reported in Mahmoud et al. [77, 78]. While in the second technique described here, the feasibility of high-frequency ultrasound to reconstruct high-resolution 3D surface images of human jawbone is investigated. The system design and experimental set-up, in addition to the required signal/image processing procedures are explained. Then, the in vitro results of the new ultrasound modality is discussed and compared with the commonly used dental $\mathrm{X}$-ray images and the real anatomy.

\subsection{Non-Invasive Assessment of Human Jawbone Using Ultrasonic Guided Waves}

Ultrasonic guided waves are sensitive to changes in microstructural properties and thus have been widely used for noninvasive material characterization. Using these waves may provide means for early diagnosis of marrow ischemic disorders via detecting focal osteoporotic marrow defect, chronic nonsuppurative osteomyelitis, and cavitations in the mandible (jawbone). Guided waves propagating along the mandibles may exhibit dispersion behavior that depends on material properties, geometry, and embedded cavities. In this work, we present the first study for the experimental analysis of guided wave propagation in jawbone. Semianalytical, finiteelement (SAFE) method was used to analyze dispersion behavior of guided waves propagating in human mandibles in Mahmoud et al. [77]. The experimental setup for the guided waves experiment is described. The results from both numerical analysis 
and guided waves experiment exhibit variations in the group velocity of the first arrival signal and in the dispersion behavior of healthy and defected mandibles. These results shall provide means to noninvasively characterize the jawbone and accurately assess the bone mechanical properties.

\subsubsection{Experimental Measurements}

The experimental set up shown in figure 3.1 was used to generate guided waves in human mandible in vitro. Two mandibles were used in this study, healthy and defected dentate dried cadaver mandibles. The defected mandible has four small holes on the anterior surface between the transmitter and the receiver. They are equally separated on the anterior surface and have diameters of $4,2,1$, and $1 \mathrm{~mm}$. The theoretical modeling of these mandibles was presented in Mahmoud et al. [77, $78]$ to compare with the experimental results.

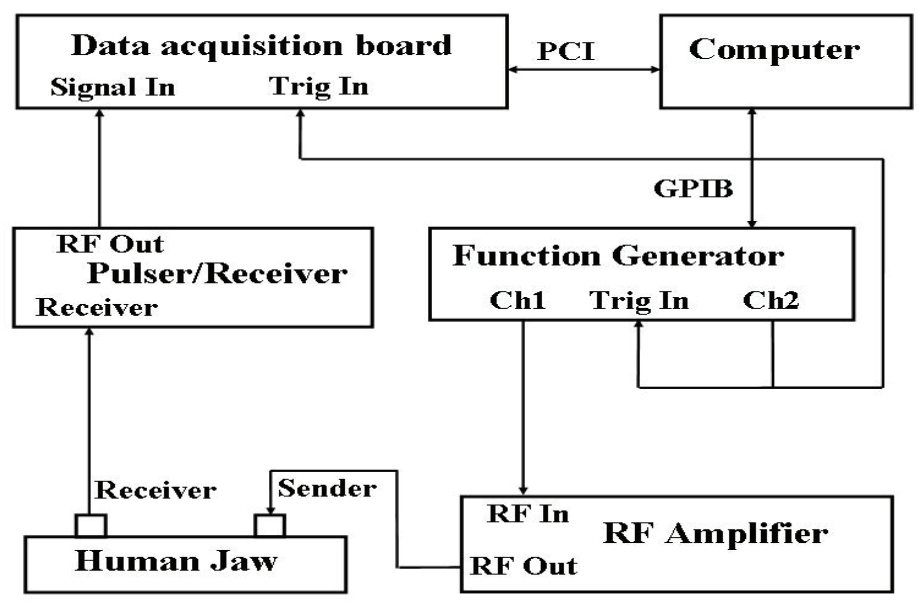

Figure 3.1 Block diagram for the experimental set up.

All the mandibles used in this study satisfy the requirements of the health insurance portability and accountability act (HIPPA). A 30-cycles sinusoidal signal was used to excite the ultrasound transmitter. A sinusoidal signal was used to generate a narrow band of frequencies around the excitation frequency. This signal was synthesized using a computer controlled function generator with 1-GHz sampling rate and 14-bit amplitude resolution (Model AFG3102, Tektronix Inc., Beaverton, OR, USA), and amplified by 75-W RF power amplifier (Model 75A250A, Amplifier 
Research, Souderton, PA, USA). The transmitter and the receiver are placed on the anterior surface of the mandible at the molar region. They were placed horizontally $20 \mathrm{~mm}$ a part. So, the ultrasound waves propagated $20 \mathrm{~mm}$ from the transmitter until reaching the ultrasound receiver which was connected to a general purpose ultrasonic pulser-receiver. Both the transmitter and receiver have $1 \mathrm{~cm}$ element diameter with 1 $\mathrm{MHz}$ center frequency, and are used to convert the electrical energy into acoustical and vice versa (Valpey Fisher Corp., Hopkinton, MA, USA). The received RF signal was then amplified and filtered by the general purpose ultrasonic pulser-receiver (Model 5900, Olympus NDT Inc., Waltham, MA, USA). Filtered and amplified signal was then fed to a high speed waveform digitizer which was synchronized with the excitation signal. A band-pass filter of $( \pm 0.1 \mathrm{MHz})$ around the excitation center frequency could be applied to remove frequency components outside the region of interest. Multiple experiments, each at a specific excitation frequency, were performed for each case of jawbone (healthy and defected). Different excitation frequencies were used to cover the range of interest $(0.7,0.8 \ldots$, and $1.2 \mathrm{MHz})$ with a $0.1 \mathrm{MHz}$ increment. The received signal at each experiment was digitized and saved with sampling rate of $15.625 \mathrm{MHz}$ as an 8-bit word using the high speed data acquisition board (Model PDA1000, Signatec Inc., Newport Beach, CA, USA).

The acquisition process was controlled and acquired by a high performance PC based system for further signal processing (Model IC1000C-C2, Signatec Inc., Newport Beach, CA, USA). All the acquisition parameters and the wavelet contour calculations are controlled, calculated and displayed by a custom user-friendly computer program designed with Labview 8.2 (National Instruments, Austin, TX, USA). The following section illustrates the use of wavelet transformation for calculating the dispersion behavior of guided wave propagation in human mandibles.

\subsubsection{Wavelet Analysis}

The displacement-signal energy is not uniformly distributed across the modes or along a single mode for a specific plate thickness. Thus, the value of group-velocity curves by themselves is somewhat limited for the acoustic guided wave practitioner. However, the wavelet transform (WT) of an acoustic signal can provide very useful information because it can describe which modes carry significant energy as a 
function of both time and frequency [79]. Hence, the wavelet contours can be used as a representation for the dispersion behavior.

The wavelet transform used in this work was the Gabor wavelet which is based on the Gaussian function. Gabor wavelet has been widely used in the field of acoustic emission because of the small window in the time as well as in the frequency domain that it can provide [79], [80]. The mother wavelet and its Fourier transform are given as:

$$
\begin{gathered}
\Psi(t)=\pi^{-\frac{1}{4}}\left(\frac{\omega_{p}}{\gamma}\right)^{\frac{1}{2}} \exp \left[-\frac{t^{2}}{2}\left(\frac{\omega_{p}}{\gamma}\right)^{2}+i \omega_{p} t\right], \\
\hat{\Psi}(\omega)=(2 \pi)^{\frac{1}{2}} \pi^{-\frac{1}{4}}\left(\frac{\omega_{p}}{\gamma}\right)^{\frac{1}{2}} \exp \left[-\frac{t^{2}}{2}\left(\frac{\omega_{p}}{\gamma}\right)^{2}\left(\omega-\omega_{p}\right)^{2}\right] .
\end{gathered}
$$

Here, $\omega_{p}$ is the center frequency and $\gamma$ is a constant taken as $\gamma=\pi(2 / \ln 2)^{1 / 2}=5.336$ as in [81].

In this work, the guided wave signals arising from human jawbone were measured experimentally in vitro at different transmitting frequencies. The received ultrasound signal at each excitation was saved. Then wavelet transformation was applied to each signal to obtain the two-dimensional wavelet contour. The wavelet contour shows the magnitudes of wavelet transform (wavelet coefficients) autoscaled to selected color map. All these contours were normalized to their highest wavelet coefficient, and then they were added together to form the final wavelet contour. Maximum frequency of $2 \mathrm{MHz}$, window size of 200 samples, and $20 \mathrm{kHz}$ frequency resolution were used as the wavelet parameters. These parameters were sufficient to provide enough data for calculating the experimental dispersion spectra for each signal. The final contour carries important information about the dispersion behavior of the guided waves. Two final contours were calculated, one for each case of jawbone (healthy and defected). These contours carry important information about the arrival time of different wave modes, and hence the group velocities can be extracted. These experimental dispersion spectra can be compared with theoretically calculated arrival times of different modes. 


\subsubsection{Results and Discussion}

Different RF signals were collected from healthy and defected human mandibles using different excitation frequencies. We repeated the experiment for each excitation frequency to cover the range $(0.7-1.2 \mathrm{MHz})$ with $0.1 \mathrm{MHz}$ increment. Figure 3.2(a) shows an example of a typical RF signal received at a specific excitation frequency. This signal was received when 30 cycles $1 \mathrm{MHz}$ tone burst excitation frequency was used through a healthy jaw sample. The signal was filtered by finite impulse response filter of bandwidth $\pm 0.1 \mathrm{MHz}$ about the excitation frequency. The time-domain signal indicates the presence of different wave modes, and the first arrival signal (FAS) was detected at $7.36 \mu$ s. Since the wave packets are not separated, it is difficult to measure the group velocities of the modes in time-domain unless the propagation distance between transmitter and receiver is increased. The frequency spectrum of the raw signal (figure 3.2(b)) shows that the effective frequency components are centered about the excitation frequency $\pm 0.1 \mathrm{MHz}$. Similar behaviors were observed in the spectra when using other excitation frequencies.

The wavelet contour shown in figure 3.3 combines both the time-domain and frequency-domain responses in a healthy mandible. The wavelet contour for the received signal at each excitation was calculated, normalized, and then all the contours were added together to form the final contour. Three lines representing the theoretically calculated modes arrival time are superimposed on the wavelet contour (figure 3.3) at frequencies of 0.9, 1 , and 1.1 MHz [77]. Note that these lines represent the arrival times of all possible modes propagating at $0.9,1$, and $1.1 \mathrm{MHz}$ center frequencies. The behavior of the wavelet coefficients within the contour looks like a cloud which means the existence of several modes within the range of frequencies $(0.7-1.2 \mathrm{MHz})$. The experimental contour can be compared with the theoretically calculated arrival time at three different frequencies which confirm the existence of several modes within the same ranges. The arrow shown on the wavelet contour (Figure 3.3) is pointing to the first arrival signal (FAS) at $1 \mathrm{MHz}$. The time is equal to $7.36 \mu \mathrm{s}$ which corresponds to $2717 \mathrm{~m} / \mathrm{s}$ group velocity calculated using the time-offlight technique for $20 \mathrm{~mm}$ distance [82]. The theoretical arrival time of the first mode is $7.45 \mu \mathrm{s}$, which corresponds to a group velocity of $2685 \mathrm{~m} / \mathrm{s}$. An error of $1.2 \%$ exists between the experimental and the theoretical results. Also, it was noticed that the 
darkest point on the wavelet contour representing high strength modes at $1 \mathrm{MHz}$ exists about $18.56 \mu$ s arrival time, which corresponds to $1077 \mathrm{~m} / \mathrm{s}$ group velocity.

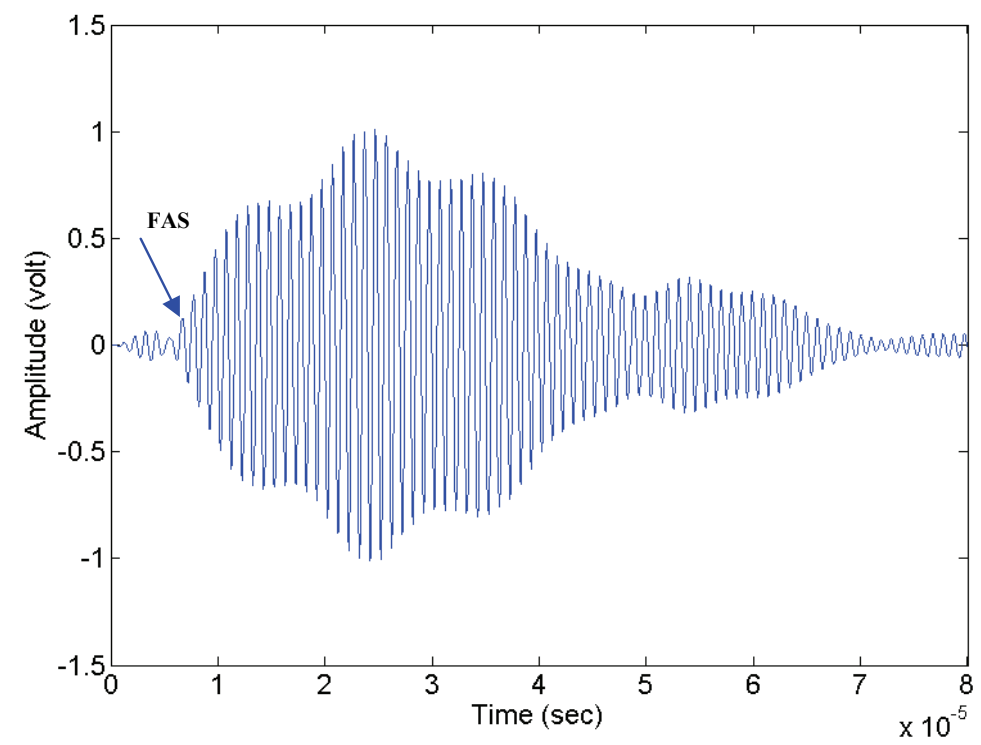

(a)

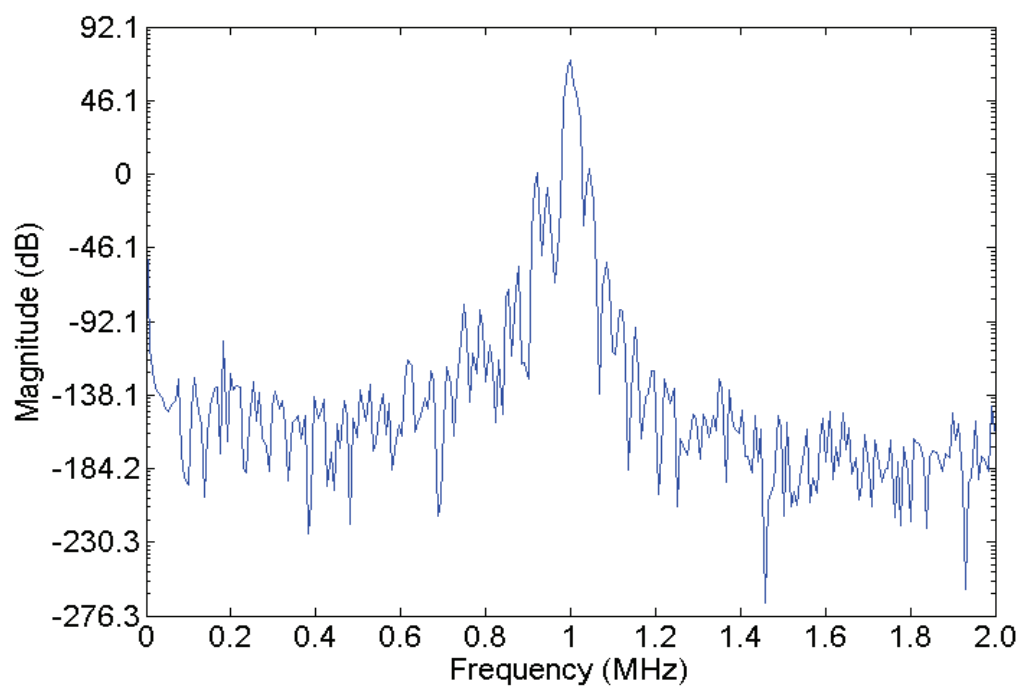

(b)

Figure 3.2 Typical RF signal received at $1 \mathrm{MHz}$ excitation frequency. a) time-domain signal filtered by band pass filter $(0.9-1.1 \mathrm{MHz})$, and b) frequency spectrum of the raw signal. 


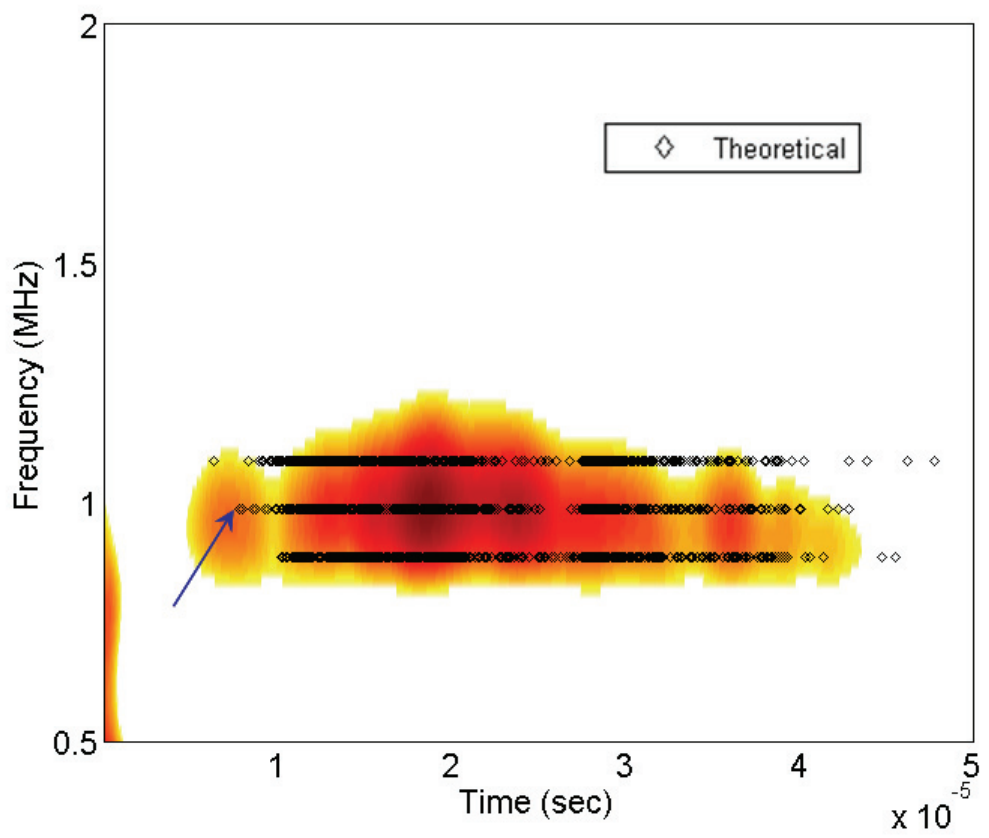

Figure 3.3 Wavelet-based experimental dispersion behavior in the high frequency range $(0.7 \mathrm{MHz}-1.2 \mathrm{MHz})$ for the healthy human mandible VS the theoretically calculated modes arrival time at $0.9,1$, and $1.1 \mathrm{MHz}$.

Figure 3.4 shows the wavelet contour for the defected mandible case. Also, three lines representing the theoretically calculated arrival time are superimposed on the wavelet contour at frequencies of $0.9,1.0$, and $1.1 \mathrm{MHz}$. The theoretical arrival times were calculated using elastic constants of $90 \%$ of that used for healthy bone tissues [78]. Matching between theoretical and experimental arrival times could provide initial assessment of the mechanical properties of the bone. In this figure, the general behavior of wavelet coefficients exhibit a main cloud with a small portion separated on the left side of the contour. There are many differences that can be observed between the two wavelet contours for the two cases (healthy and defected). By comparing the two contours, it is clear that the cloud in the defected case is shifted to the right and the arrival time range becomes smaller. Moreover, the intensity of wavelet coefficients has decreased within the cloud, which indicates that the modes become weaker comparable with the healthy mandible case. Also, the experimental arrival time of the first mode for the defected case (defined by the arrow) has increased to $11.33 \mu \mathrm{s}$. The corresponding group velocity has decreased to $1765 \mathrm{~m} / \mathrm{s}$. 


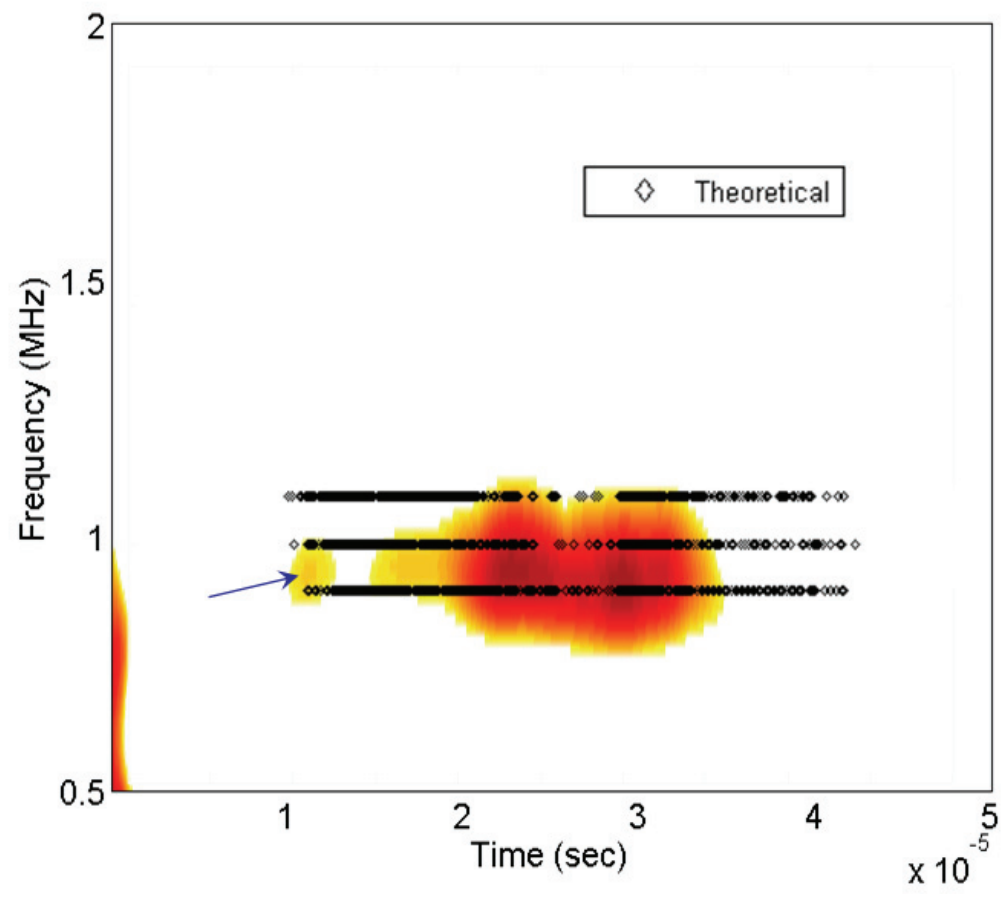

Figure 3.4 Wavelet-based experimental dispersion behavior in the high frequency range (0.7 MHz-1.2 MHz) for the defected human mandible VS the theoretically calculated modes arrival time at $0.9,1$, and $1.1 \mathrm{MHz}$.

The theoretical arrival for the first mode is recorded at $10.89 \mu \mathrm{s}$ which corresponds to $1836 \mathrm{~m} / \mathrm{s}$ group velocity which is comparable with the experimental one $(1765 \mathrm{~m} / \mathrm{s})$ with $4 \%$ difference.

One of the main diagnostic tools for the detection and treatment of periodontal disease is radiographic analysis. Current radiographic analysis, however, has limitations when it comes to reliability. Comparisons of clinical and radiographic measurements of inter-proximal vertical defects reveal that the traditional standardized intra-oral radiograph underestimates bone level and defects by approximately $1.4 \mathrm{~mm}$ [83]. It has also been noted that significant attachment loss has preceded standard radiographic bone loss by 6-8 months [84]. The use of this new technology may be more sensitive with the added advantage of no radiation.

In this study, only the high frequency range $(0.7-1.2 \mathrm{MHz})$ was considered in the experimental process, since $1 \mathrm{MHz}$ center frequency transducers were used. These transducers are more common and available in our laboratory than those of lower 
frequencies. Moreover, it is difficult to use commercial low frequency transducers due to their large geometry and low availability. It was observed that it is difficult to find two identical mandibles since there are different factors affecting mandibles properties like age, sex, weight...etc.

The defected jawbone was modeled as a $10 \%$ change in the mechanical properties rather than using holes in the model to avoid difficulties in the implementation. In general, there are some errors when comparing the experimental and theoretical results. The main source of error that affected this study is the complicated geometry of the jawbone that made both numerical and experimental procedures harder. Also, another significant source of error between experiments and modeling is that the values of the model material and mechanical properties are taken from the literature and do not correspond to the actual sample properties. Moreover, there are other undefined reasons for this difference in the velocities, but several reasons may account for this discrepancy, such as the transducer geometry and type, coupling, and test geometry differences which may affect the distribution of the acoustic energy [85]. 


\subsection{High Frequency 3D Echodentographic Imaging Modality for}

\section{Early Assessment of Periodontal Diseases: In Vitro Study}

Currently, there is a lack of imaging modalities to accurately predict minute structures and defects in the jawbone. Most significant has been the inability of 2D radiographic images to detect bony periodontal defects resulting from periodontal infections. This study investigates the feasibility of utilizing high-frequency ultrasound to reconstruct high resolution images for human jawbone. The system employs high frequency single-element ultrasound focused transducers (30-60 MHz) for scanning. Different frames are reconstructed $500 \mu \mathrm{m}$ apart for the 3D reconstruction. Signal processing algorithms are applied on the received ultrasound signals for filtering, focusing, and envelope detection before frame reconstruction. Then edge detection is adopted to detect the bone surface in each frame. All edges are combined together to render a 3D surface image of the jawbone. In vitro results of dentate and non-dentate human mandibles elucidate the ability of the ultrasound imaging system to detect changes in small structures. Moreover, comparisons with the anatomy and X-ray images assured the system efficacy. These results suggest that the high frequency ultrasound system shows great potential in providing a noninvasive method to characterize the jawbone and to detect periodontal diseases at early stages.

\subsubsection{System Schematic}

Figure 3.5 shows a schematic diagram of the high-frequency ultrasound imaging system. The upper part explores the main component of the system. A main bang of negative impulse type is used as the excitation signal for the ultrasound transducer. This signal is generated and amplified using a general purpose ultrasonic pulserreceiver (Model 5900PR, Olympus NDT Inc., Waltham, MA, USA). The excitation signal is synchronized with the data acquisition trigger input using a common signal generated by a computer controlled function generator with a $1-\mathrm{GHz}$ sampling rate and 14-bit amplitude resolution (Tektronix Inc., Beaverton, OR, USA). The same ultrasound transducer is used as both ultrasound transmitter and receiver and is 


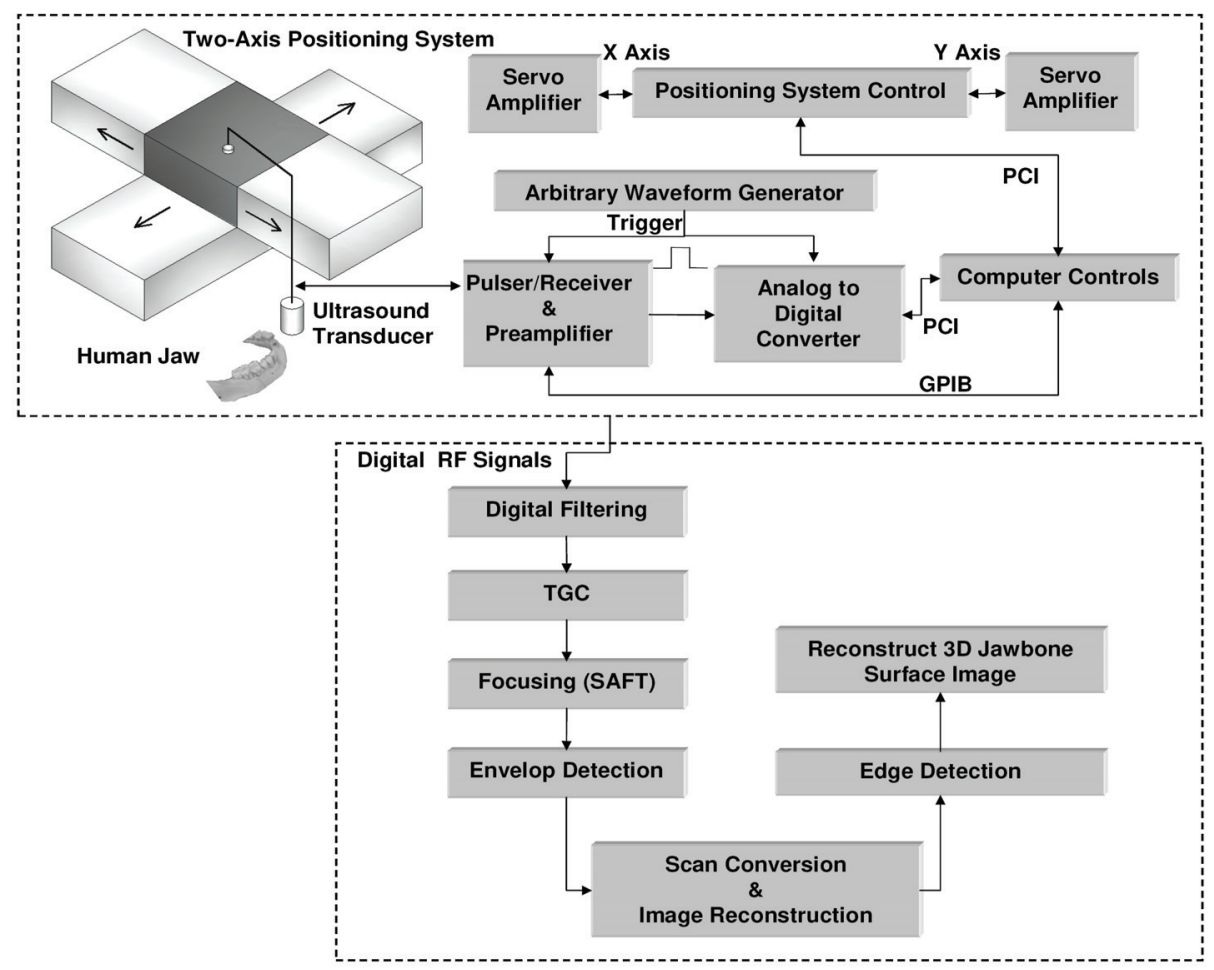

Figure 3.5 Schematic diagram showing the design and processing steps of the highfrequency ultrasound imaging system for jawbone.

connected to transmit/receive the port of the ultrasound pulser-receiver, which is operated in the pulse-echo mode. Two single-element ultrasound transducers are used (30 and $60 \mathrm{MHz}$ ). This echo signal is then amplified and filtered by the ultrasonic pulser-receiver. The filtered and amplified signal is fed to a high-speed waveform digitizer which is synchronized with the excitation signal. The imaging system uses a high performance PC based system for system control, synchronization, and for further signal processing (Signatec Inc., Newport Beach, CA, USA). The PC controls a two-axis (two translational) precision positioning system with $1 \mu \mathrm{m}$ resolution (Danaher Corp., Washington, DC, USA). The positioning system is synchronized with the data acquisition to collect the ultrasound signals continuously on the fly during the transducer movement down to $10 \mu \mathrm{m}$ apart. The received RF signals are digitized and saved using sampling rates of 62.5-250 MHz according to the transducer center frequency. Signals are acquired and saved as an 8-bit word using a high-speed data acquisition board (Signatec Inc., Newport Beach, CA, USA). This high speed data acquisition board is fully controlled with the main PC via the PCI bus. A custom 
user-friendly computer program designed with Labview 8.2 (National Instruments, Austin, TX, USA) is used to control both the data acquisition board and the positioning system. Raw RF signals are collected from the field of view (FOV) during the transducer movement. These signals are saved sequentially according to the lateral location for further post-processing. During the 3D acquisition, the positioning system moves the transducer further steps in the elevation direction and the lateral scanning process is repeated. The system can be programmed with various scanning profiles, and for each profile the operator shall determine the FOV parameters in the axial, lateral, and elevation directions. Also, the operator is able to determine the lateral acquisition speed that affects distance between RF signals, and the elevation step size. After completing the acquisition process, the data is transferred to Matlab 7.1 (The MathWorks, Inc., Natick, MA, USA) for post processing and image reconstruction.

\subsubsection{Signal and Image Processing}

The lower part of Figure 3.5 describes the processing steps applied to reconstruct the final ultrasound image. Raw signals are filtered using a band-pass filter with a pass-band of $\pm 20 \%$ of the transducer center frequency to remove the noise from the raw data. Time gain control (TGC) is applied after filtration to compensate for the attenuation effect using simple linear functions. The focusing procedure is then applied using the weighted synthetic aperture focusing technique (SAFT), which has been widely utilized for single-element systems, and is described in detail in the next chapter. This procedure improves the image resolution via broadening the main lobe and lowering the sidelobes of the point spread function. After applying SAFT, a signal processing algorithm is applied to extract the signal envelop by calculating the amplitude of the Hilbert transform of the echo signal. Logarithmic compression is then applied to reduce the dynamic range for visualization. Finally, images are linearly mapped to gray scale levels for display at the proper dynamic range such as $80 \mathrm{~dB}$. In these B-mode images, the outer boundary of the jawbone appears brighter than any other region. This brightness is resulted from the high reflection coefficient in the tissue-bone interface that causes strong reflections. The jawbone boundary for each image is detected via applying image thresholding algorithm followed by edge detection. The arrival time of the first boundary (bone surface) for each image line 
then is recorded. Arrival times are saved in a two dimensional matrix as a row for each image boundary. Each row represents a lateral scanning and each column represents the elevation direction. A 3D surface image is then reconstructed by plotting the recorded arrival times, which represent the jawbone boundary, within the ultrasound scanning area. A cubic smoothing spline function is then utilized to smooth the 3D mesh, before displaying the 3D surface image of the jawbone surface.

\subsubsection{Results and Discussion}

This study investigates the ability of using a non-invasive high frequency singleelement ultrasound imaging system for reconstructing three dimensional bone surface images of the human jaw using high resolution B-mode images. This technique is the first to provide a simple and efficient tool for the diagnosis of moderate to advanced periodontal diseases. Periodontal bony defects may exhibit horizontal and/or vertical components. Edentulous and dentate dry cadaver mandibles with both bony periodontal defects were used in the study. All the mandibles used in this study satisfy the requirements of the Health Insurance Portability and Accountability Act (HIPAA). Twenty-four scans (frames) were acquired for the mandible shown in figure 3.6(a), for the region highlighted by the yellow rectangle. In each frame, the RF signals were collected $45.5 \mu \mathrm{m}$ apart to cover a scan width of $25.9 \mathrm{~mm}$. During experiments, all mandibles were placed in degassed water near the transducer focus with an angle that ranges from 0-90 degrees. For the mandible shown in figure 3.6(a), an angle of 30 degrees was used and frames were acquired $0.5 \mathrm{~mm}$ apart with respect to the transducer elevation direction and starting near the tooth roots. Figures 3.6(c)-(e) show different views of the 3D surface image for the rectangular region described in figure 3.6(a). On the other hand, figure 3.6(b) shows the corresponding X-ray image for the right anterior side. Notice that the topography of the three landmarks matches the anatomy of the mandible shown in figure 3.6(a). Landmark \#1 describes a horizontal bony defect between the two premolars. Landmark \#2 shows the distinct image of the mental foramen, one of the major landmarks of the jawbone. Landmark \#3 describes a severe vertical bony defect adjacent to the distal root of the first molar. $\mathrm{X}$-ray images are not showing the elevation direction, since images describe the 


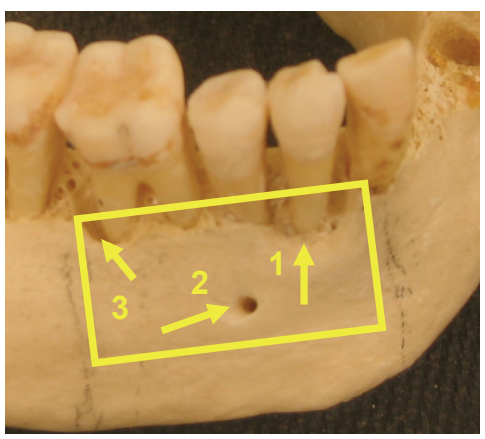

(a)

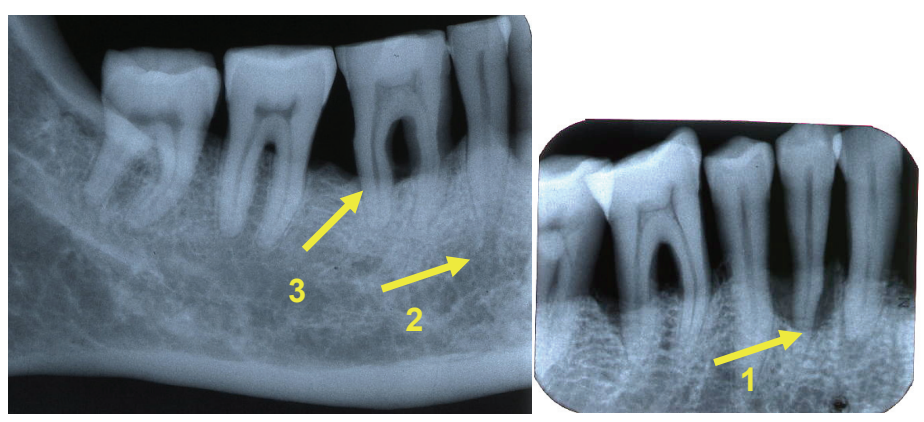

(b)

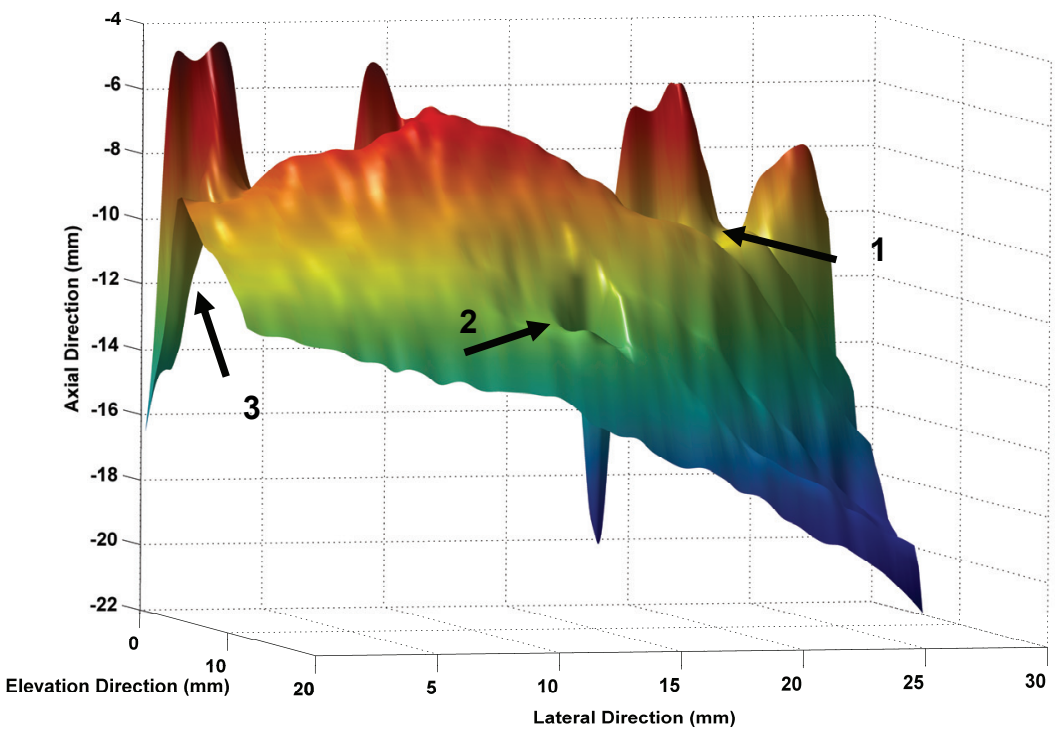

(c) 


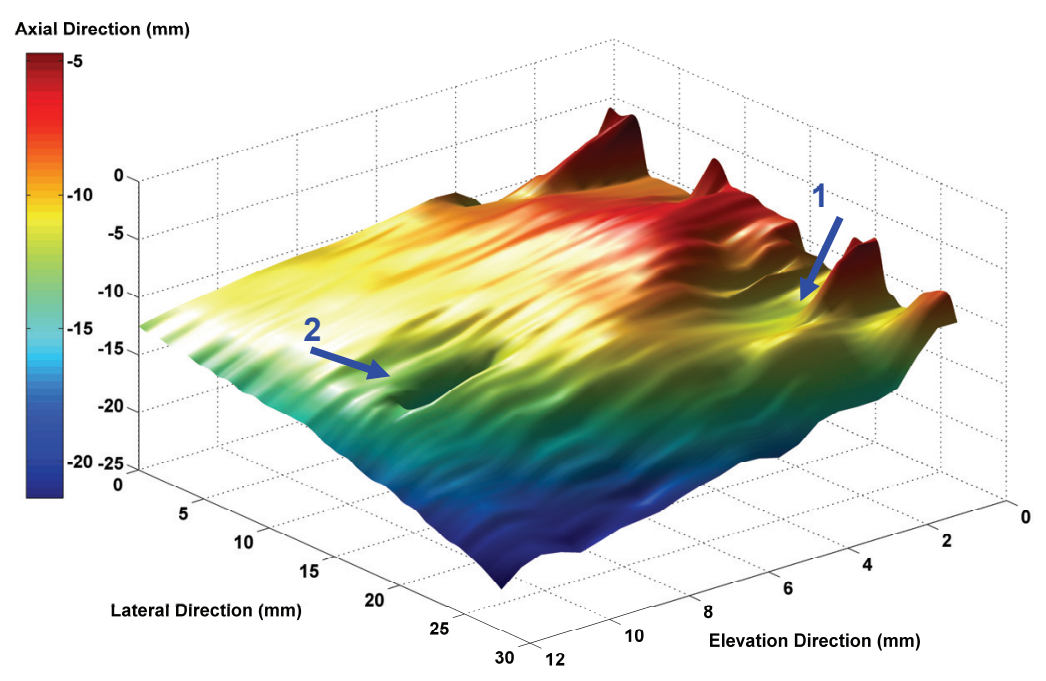

(d)

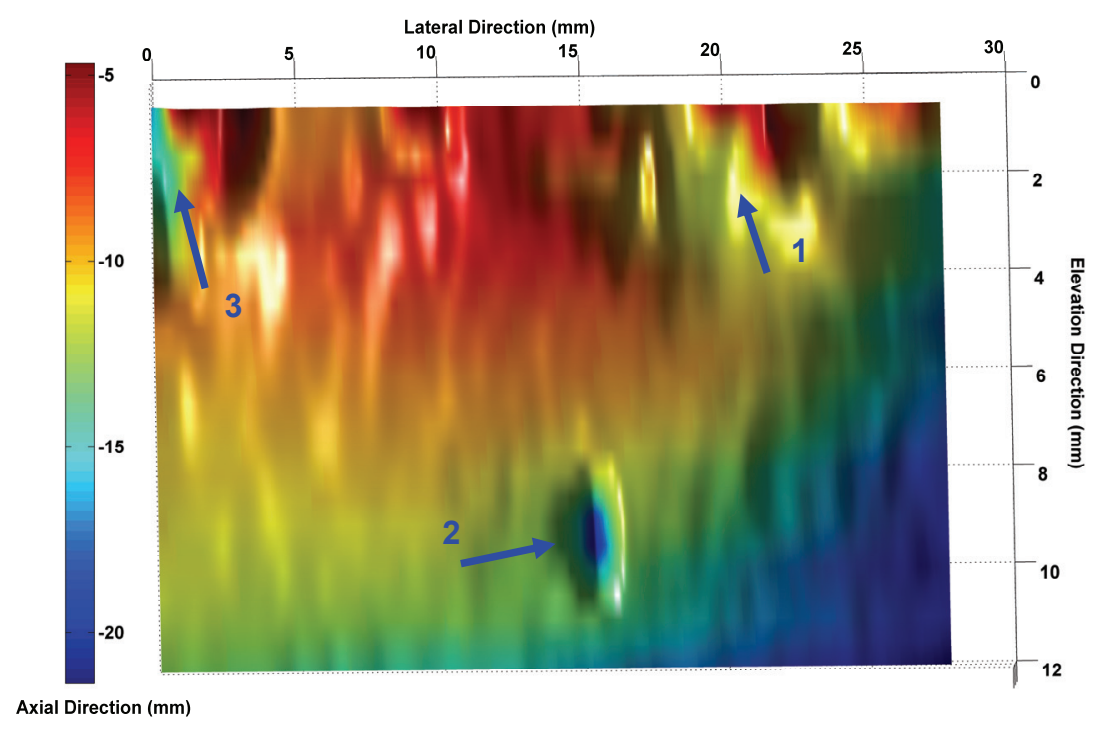

(e)

Figure 3.6 Defected dentate dried cadaver mandible. a) Photographic image for the mandible with a rectangle showing the scanned region and three landmarks, b) the corresponding X-ray radiographic images showing landmarks \# 1, 2 and 3, and (c)-(e) different views for the $3 \mathrm{D}$ ultrasound surface image of the jawbone surface. It is clearly observed that the X-ray images have very poor information about landmarks $\# 2$, and \#3, and limited information to perfectly describe landmark \#1. 
projection of the right side of the mandible. According to the mandible placement, this X-Ray image field should include landmarks \#2, and \#3. However, these landmarks are missed due to the projecting image. Although landmark \#1 can be identified from the X-ray images, it provides no information about the main defect (horizontal bony defect) within this region.

The ability of the new system to detect more challenging defects was tested. Figure 3.7(a) shows a photographic image of a human mandible with a moderate three-wall bony defect. The arrow, shown in figure 3.7, describes approximately the area of the defect around the distal root of the first molar. In the X-ray radiographic image of the same site (figure 3.7(b)), it is difficult, or even impossible, to detect this bony defect. On the other hand, the bony defect is described accurately in the $3 \mathrm{D}$ ultrasound bone surface reconstruction (figures 3.7(c)-(e)) of the same site, and it is possible to show its progress within this small region. In this reconstruction process, RF signals were collected $22 \mu \mathrm{m}$ apart to cover a scan width of $15 \mathrm{~mm}$, and frames were $0.5 \mathrm{~mm}$ apart. Three different views for the 3D ultrasound bone surface image are shown in figures 3.7(c)-(e). These views shall help dentists to define the defect accurately, and to make further decisions. The system was further used to reconstruct a 3D ultrasound bone surface image for a small region (15.5 mm (lateral) x $9.5 \mathrm{~mm}$ (elevation)) within a third mandible around the third molar, as described in figure 3.8(a). In this region two landmarks are defined, where landmark \#1 defines a sever three-wall bony defect and landmark \#2 describes a moderate vertical bony defect. It is obvious that the X-ray radiographic image shown in figure 3.8(b) is not able to describe the defect at landmark \#2, nor was able to provide useful information about landmark \#2. These defects at the two landmarks are fully described in the three views (figures 3.8(c)-(e)) of the 3D ultrasound bone surface image. In this

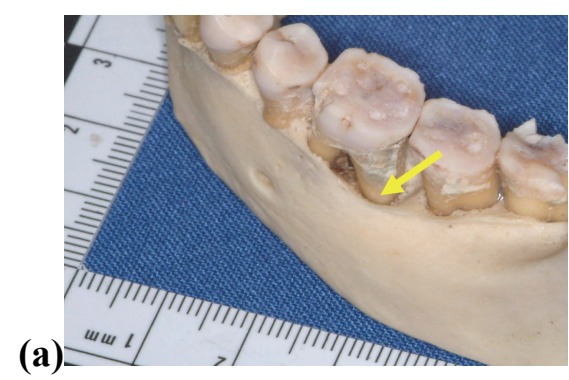

(b)

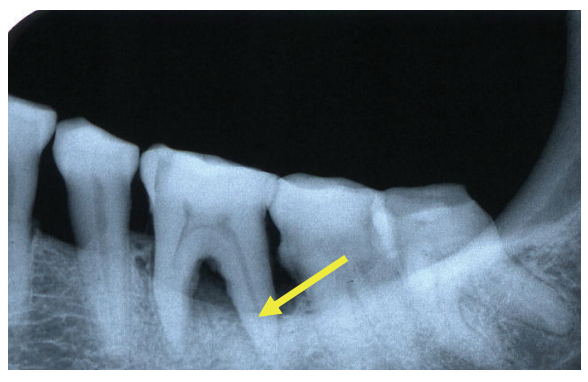



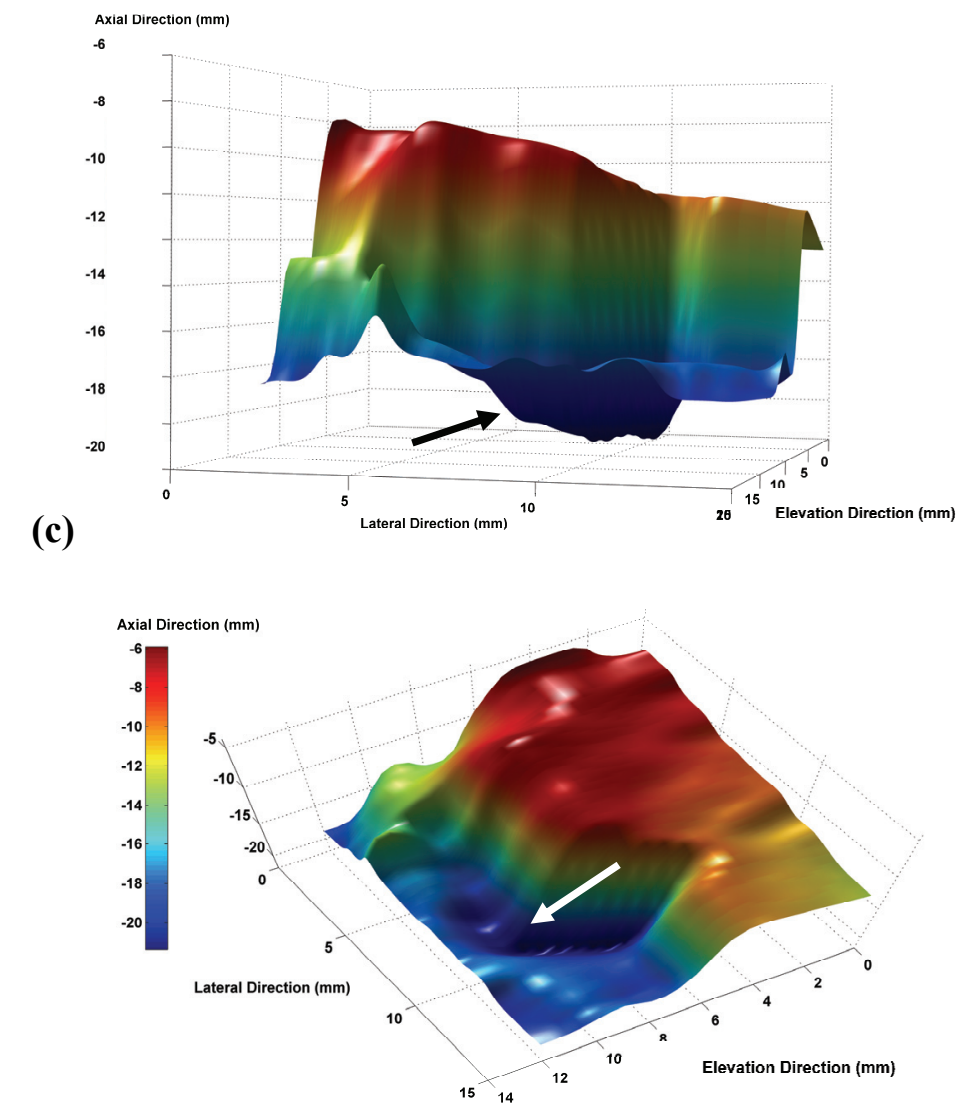

(d)

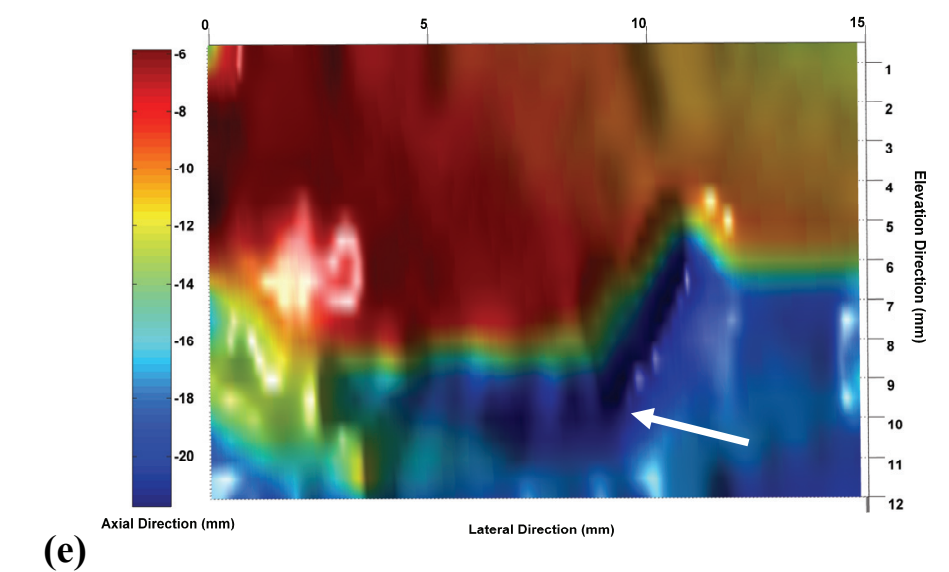

Figure 3.7 Defected dentate dried cadaver mandible. a) Photographic image for the mandible with an arrow showing a bony defect adjacent to the distal root of the first molar, b) the corresponding X-ray radiographic image with an arrow describing the region of defect, and (c)-(e) different views for the 3D ultrasound surface image of the jawbone surface. It is clearly observed that the X-ray image provides no information about the bony defect described by the arrow. 


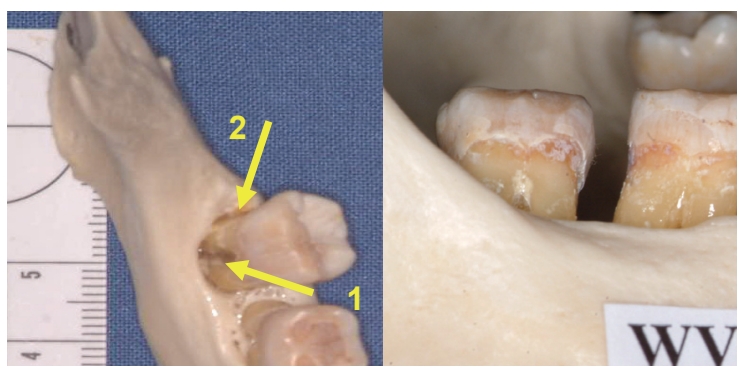

(a)

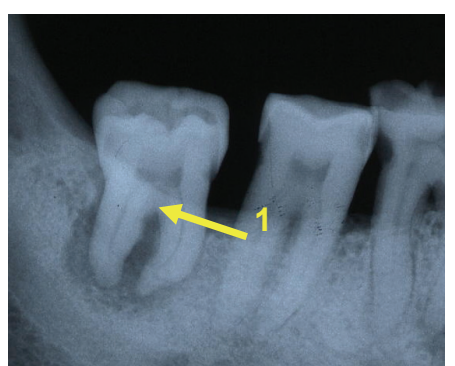

(b)

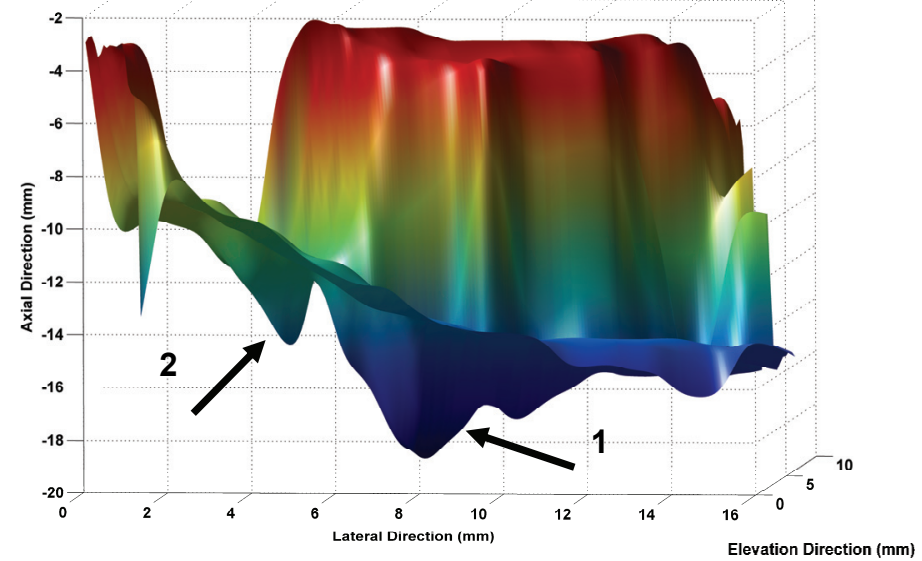

(c)

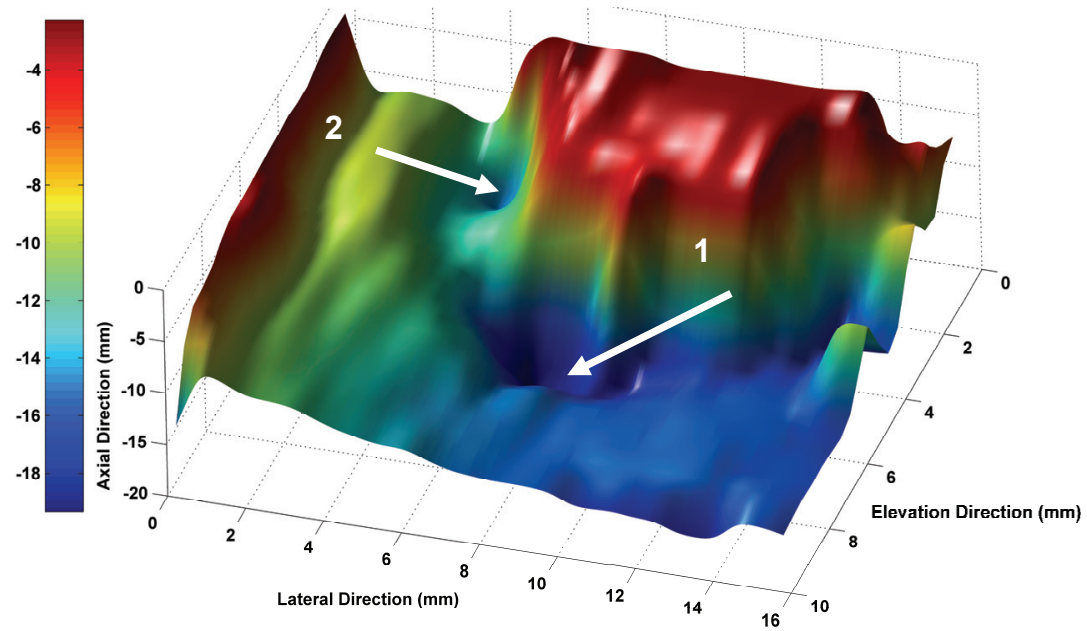

(d) 


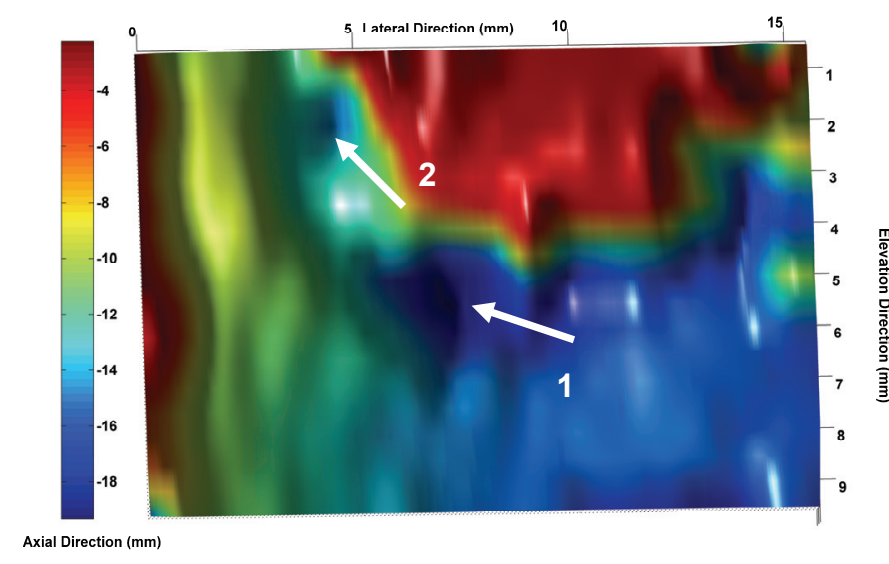

(e)

Figure 3.8 Defected dentate dried cadaver mandible. a) Two Photographic images for the mandible with arrows showing two landmarks around the third molar, b) the corresponding X-ray radiographic image with an arrow describing landmark \#1, and (c)-(e) different views for the 3D ultrasound surface image of the jawbone surface. The 3D ultrasound images were able to detect different bony defects which couldn't be defined using X-ray images at landmark \#1 (sever defect), and landmark \#2 (early stage defect).

reconstruction. RF signals were collected $25 \mu \mathrm{m}$ apart to cover a scan width of 15.5 $\mathrm{mm}$, and frames were $0.5 \mathrm{~mm}$ apart.

The aim of this pilot study is to elucidate the usefulness of this high-resolution 3D ultrasound imaging technique to image opaque periodontal bony defects. Since a dry mandible may not be the ideal specimen for in vivo representation of human mandibles, which include gingivae, it is important to assess the performance of our proposed technique by testing a mandible with gum-tissue mimicking material. A thin slice of meat was attached to a dry mandible, with sever 3-wall bony defect, around the second molar. Ultrasound scanning was performed, for a small region around the defect, on the mandible after attaching the tissue as shown in figure 3.9(a). Fifteen scans, or B-mode images, were acquired $0.5 \mathrm{~mm}$ apart in the elevation direction, while the RF signals were collected $44 \mu \mathrm{m}$ apart in the horizontal direction. The experimental set-up was similar to the previous experiment for dry jaw. The 
signal processing procedures have been adapted via modifying the thresholding algorithm to detect the bone surface under the tissue. Figure 3.9(a) shows photographic images for the jaw before and after adding the gum-tissue mimicking layer. Three landmarks are highlighted within the scanned area around the defect. Landmark \#1 describes the root of the second molar, where \#2 describes the bone line after the pocket. Landmark \#3 describes the bone edge at the end of the defect, which exists mainly between the three landmarks. The 3D ultrasound surface image of the jawbone, shown in figures 3.9(c)-(e), describes all the three landmarks. Moreover, the defect can be identified quantitatively in the three directions (lateral, axial, and elevation). Figure 3.9(b) shows the corresponding X-ray image for the same site of the mandible. Landmarks\#1 and \#2 are almost projected into the same point, which demonstrate the lack of the X-ray radiography to detect defects in the elevation direction. Moreover, it is difficult to extract information about the pocket end using Landmark \#3 shown in figure 3.9(b).
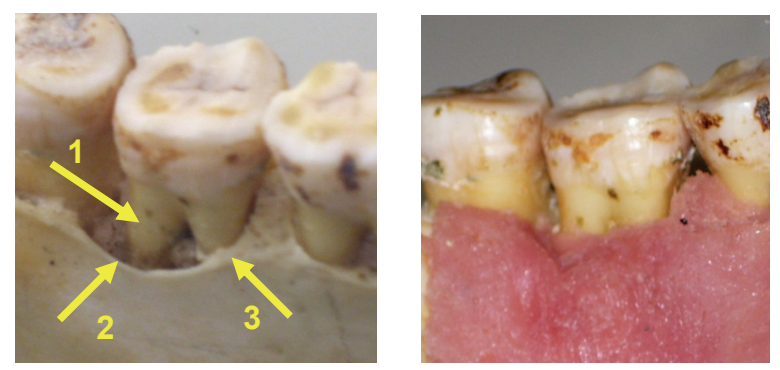

(a)

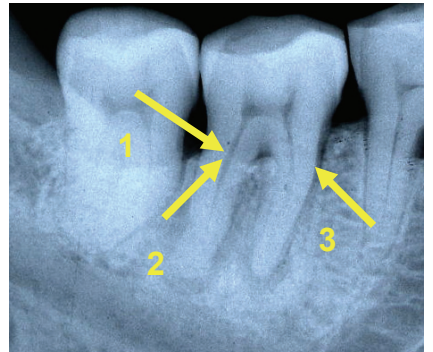

(b)

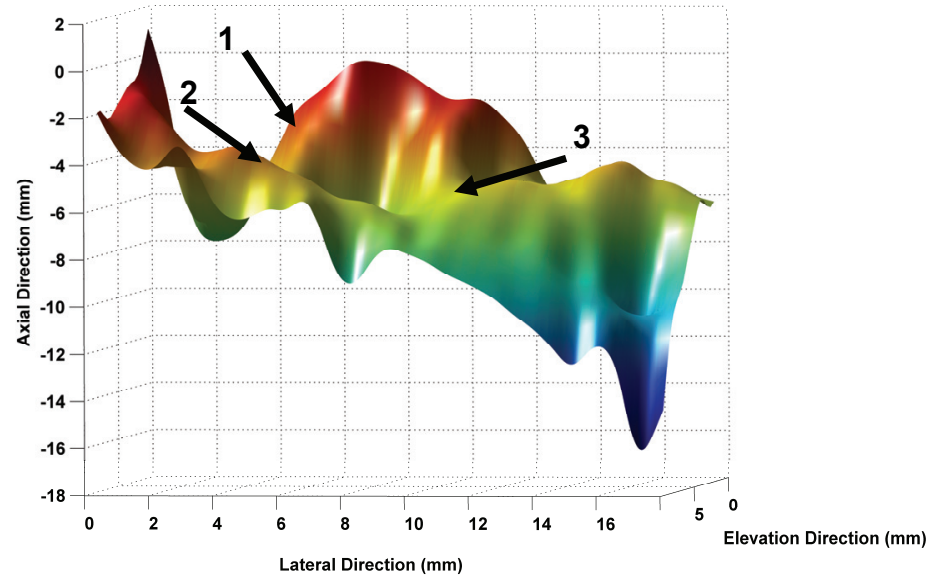

(c) 


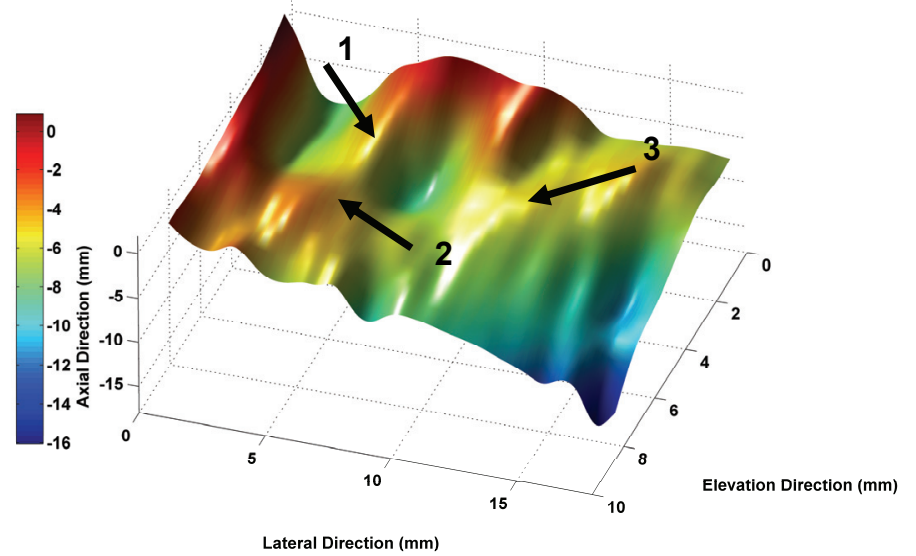

(d)

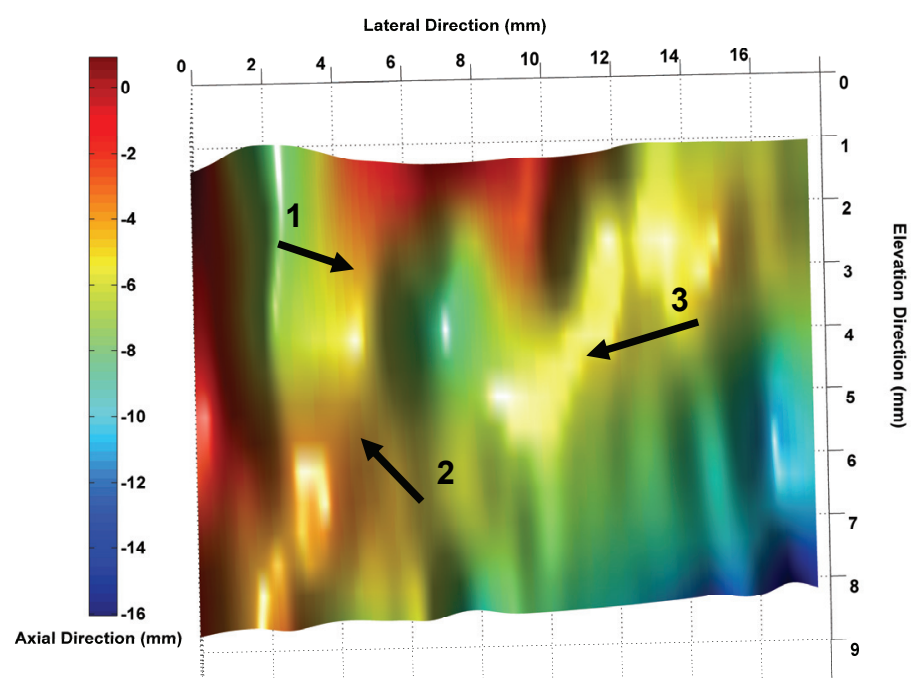

(e)

Figure 3.9 Defected dentate dried cadaver mandible with gum-tissue mimicking material. a) Photograph images for the jaw before and after adding the tissue layer showing three landmarks, b) an X-ray radiography image showing the same landmarks, and (c)-(e) different views for the 3D ultrasound surface image of the jawbone surface.

Results of this novel ultrasound modality suggest that the reconstructed 3D images can be used to describe different degrees of horizontal and/or vertical bony defects. Most helpful to the clinician is the resultant 3D rendering which can aid in not only the diagnosis but also the specific type of surgical treatment. These defects 
are difficult to detect using the current radiographic imaging modalities, as demonstrated in this chapter. 


\section{CHAPTER 4}

\section{High Frequency Precise Ultrasound Imaging System FOR SMALl ANIMALS}

This chapter describes the development and testing of a high frequency (30-120 $\mathrm{MHz}$ ) single-element ultrasound imaging system. The system uses state-of-the-art electronic components to achieve maximum accuracy via synchronizing the high-

frequency data acquisition with the high-precision movements of the positioning system. Various signal processing techniques were adopted for filtering, focusing, and reconstructing the initial ultrasound B-mode images. Furthermore, multiple advanced image processing techniques were applied to obtain final B-mode images of high quality. This system is capable of providing high-resolution B-mode images of small animals such as mouse hearts and blood vessels that can be used for quantitative measurements. Also, the system can perform various types of scanning precisely using variable FOV (1 up to $250 \mathrm{~mm}$ ). The focusing quality and the system performance were evaluated via imaging several wire phantoms at different depths. Moreover, the system was used to reconstruct B-mode images for isolated mouse hearts and blood vessels of different models in vitro. The technical details regarding the system design and performance are explained, and then results are presented followed by the discussion.

\subsection{Methods}

\subsubsection{System Schematic}

The upper part of the block diagram shown in figure 4.1 explains the main components of the high-frequency ultrasound imaging system for small animals. A main bang of negative impulse type is used as the excitation signal for the ultrasound transducer. This signal is generated and amplified using general purpose ultrasonic pulser-receiver (Model 5900PR, Olympus NDT Inc., Waltham, MA, USA), which is used as a transmitter. Typical no-load amplitude at the transmitter output of 175 volts 


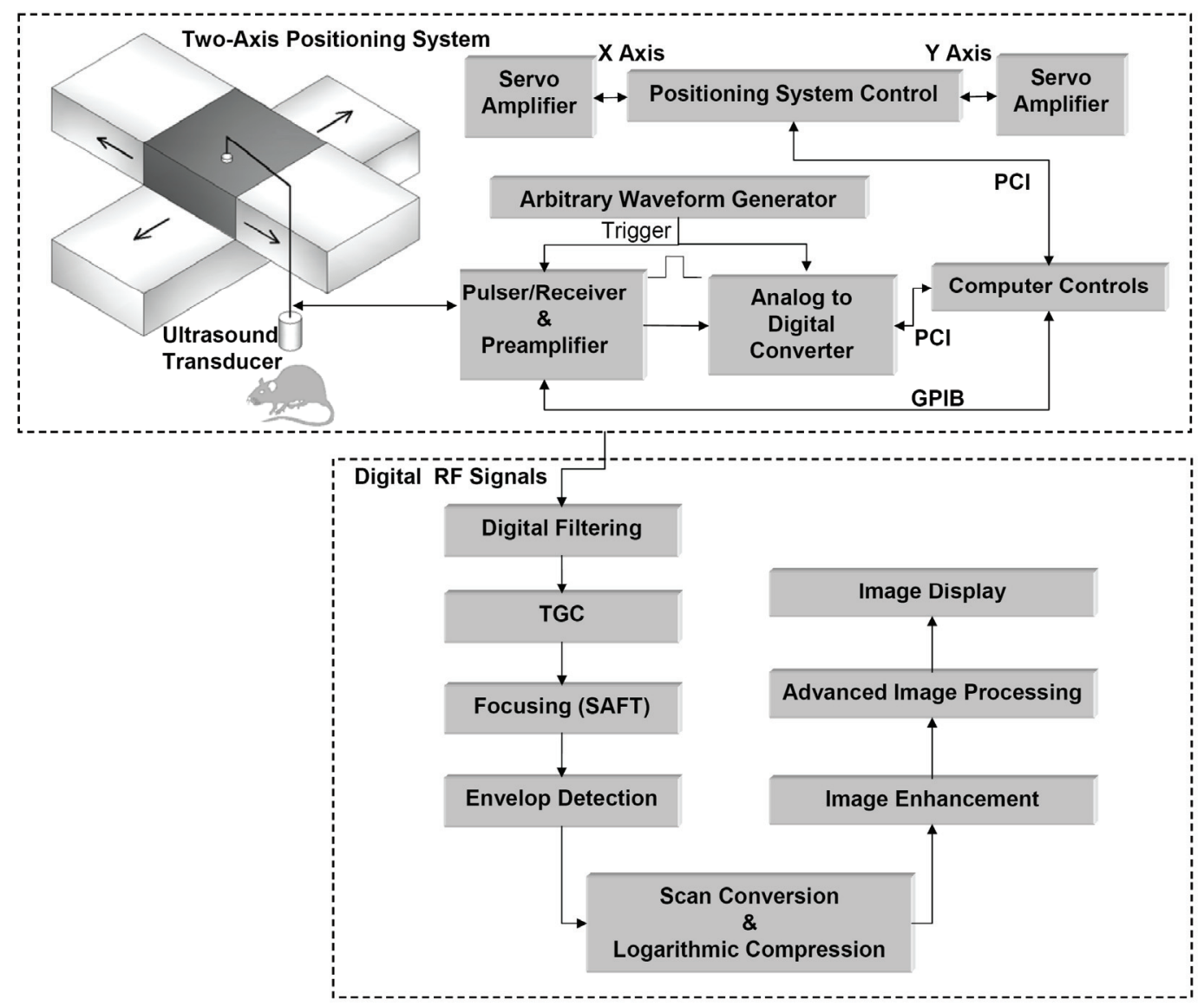

Figure 4.1 Block diagram of the high-frequency ultrasound system for small animal imaging. System design and processing steps are shown.

is used for the main bang with rise-time of 2 ns. These properties of the excitation signal are sufficient to stimulate the ultrasound transducers used with the system in the wide range of frequencies (30-120 MHz). The IEEE-488 (GPIB) standard interface could be used to control and communicate with the ultrasonic pulserreceiver. The excitation signal is synchronized with the data acquisition trigger input using a common signal generated by a computer controlled function generator with 1GHz sampling rate and 14-bit amplitude resolution (Model AFG3102, Tektronix Inc., Beaverton, OR, USA). The same ultrasound transducer is used as both ultrasound transmitter and receiver and is connected to transmit/receive port of the ultrasound pulser-receiver, which is operated in the pulse-echo mode.

Several single-element ultrasound transducers are used, which can be integrated with the system. Two ultrasound transducers of $30 \mathrm{MHz}$ and $60 \mathrm{MHz}$ in frequency 
are used (Olympus NDT Inc., Waltham, MA, USA), while the third transducer is a $100 \mathrm{MHz}$ (Valpey Fisher Corp., Hopkinton, MA, USA). The technical specifications of these three transducers are summarized in table 4.1 shown below. These transducers are used to convert the electrical energy into acoustical and vice versa. Ultrasound waves reflected from the field of view are received by the ultrasound transducer forming the desired echo. This echo signal is then amplified and filtered by the ultrasonic pulser-receiver. Filtered and amplified signals are fed to a highspeed waveform digitizer which is synchronized with the excitation signal. The imaging system uses a high performance PC based system for system control, synchronization, and for further signal processing (Signatec Inc., Newport Beach, CA, USA). The PC controls a two-axis (two translational) precision positioning system with $1 \mu \mathrm{m}$ resolution (Danaher Corp., Washington, DC, USA).

The positioning system consists of XY stages that use recirculating bearings, a linear motor and linear encoder that can provide high speed up to $2.5 \mathrm{~m} / \mathrm{s}$ combined with a good accuracy and repeatability. A motion controller (Galil Motion Control Inc., Rocklin, CA, USA) is used as the control electronics for the positioning system. The stages are powered by a pair of compact but powerful servo amplifier (Model Servostar CD, Danaher Corp., Washington, DC, USA). The positioning system control is controlled by the PC via the PCI port. The positioning system is synchronized with the data acquisition to collect the ultrasound signals continuously on the fly during the transducer movement down to $10 \mu \mathrm{m}$ apart. The transducer movement spacing affects the lateral resolution of the B-mode image. The received RF signals are digitized and saved with sampling rates up to $1 \mathrm{GHz} \mathrm{MHz}$ as an 8-bit word using a high-speed data acquisition board (Signatec Inc., Newport Beach, CA, USA). Sampling frequencies have been selected based on the Nyquist-Shannon

Table 4.1 Ultrasound Transducers Technical Description

\begin{tabular}{c|ccc}
\hline Specifications/Transducer & $\mathbf{3 0 ~} \mathbf{~ H H z}$ & $\mathbf{6 0 ~ M H z}$ & $\mathbf{1 0 0 ~ M H z}$ \\
\hline Center Frequency (MHz) & 29.63 & 58 & 99.61 \\
-6 dB Bandwidth (MHz) & 23.47 & 73.34 & 74.22 \\
Element Diameter (in.) & 0.25 & 0.125 & 0.125 \\
Focal Length (in) & 1.25 & 0.25 & 0.50 \\
\hline
\end{tabular}


sampling theorem (sampling rate $\geq 2 \times$ transducer frequency) to avoid aliasing errors. The high-speed data acquisition board is fully controlled with the main PC via the PCI bus. Most of the system components are placed and bolted on a custom designed antivibration table to minimize motion errors and to assure accurate acquisition. A custom user-friendly computer program designed with Labview 8.2 (National Instruments, Austin, TX, USA) is used to control both the data acquisition board and the positioning system. Raw RF signals are collected from the FOV during the transducer movement as shown in figure 4.2. These signals are saved sequentially according to the lateral location for further post-processing. The data is then transferred to Matlab7.1 (The MathWorks, Inc., Natick, MA, USA) for post processing and image reconstruction.

\subsubsection{Focusing and Signal Processing}

In order to obtain the final high-resolution B-mode ultrasound images in the highfrequency range, several signal and image processing algorithms are applied to the RF signals. The lower part shown in figure 4.1 describes the processing steps applied to reconstruct the final ultrasound image. Raw signals are filtered using a band-pass filter of about $\pm 20 \%$ of the transducer center frequency (i.e. for $100 \mathrm{MHz}$ the passband is $80-120 \mathrm{MHz}$ ) to remove the noise from the raw data. Time gain control

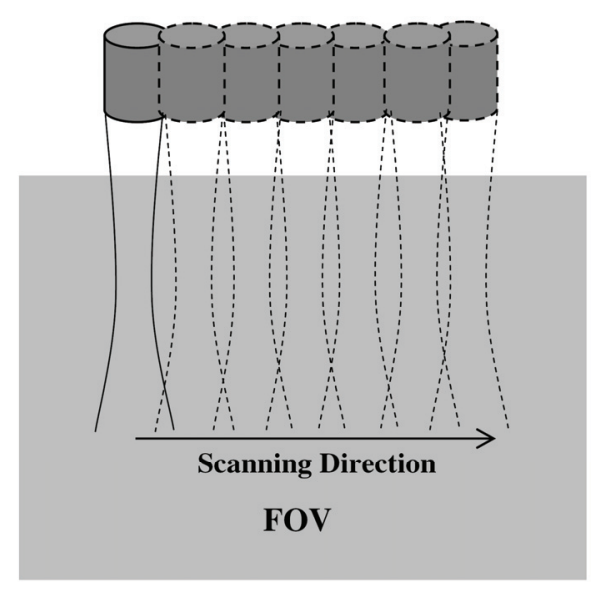

Figure 4.2 Schematic of scanning on the fly from different positions. 
(TGC) could be applied after filtration to compensate the attenuation effect using simple linear functions. The focusing procedure is then applied using the weighted synthetic aperture focusing technique (SAFT), which has been widely used for singleelement systems [86-88]. In this work, the focusing technique described by Frazier et al. [87] has been used with slight adaptation to assure homogenous focusing at various depths. It has been proposed to combine conventional B-mode and synthetic aperture imaging techniques to overcome the limited depth of field for a highly focused transducer. Moreover, it allows improving the lateral resolution beyond the transducer focus by considering the focus as a virtual element and applying synthetic aperture focusing techniques. The sketch in figure 4.3 briefly illustrates the basic concepts of SAFT [88].

SAFT considers the transducer's focal point as a virtual source, as shown in figure 4.3(a). This virtual source is assumed to produce a wave propagating both forward and backward with respect to itself that is spherical within a certain angular extent. When a linear scan is performed with the transducer, the sound field generated by the virtual source at the current position will overlap with the sound fields produced at adjacent positions. Hence, synthetic aperture focusing can be performed in the overlapping region before and after the virtual source. Figure 4.3 explains this concept; points $p_{1}$ and $p_{2}$ in figure 4.3(a) are in the overlapping region after and before the virtual source, and they can be synthetically focused using the received scan lines at which the points $p_{1}$ and $p_{2}$ are illuminated by the sound field. In figure 4.3(b) the virtual-source concept is described where the scan line $i$ is a distance $x_{i}$ away from the axis of the synthesized beam. Since the sound field of a scan line $i$ propagates through the desired focal point $p$, the echo signal from scan line $i$ can be added to the beam constructively by applying an appropriate time delay. Based on the virtualsource concept, the time delay applied to the received signal of scan line $i$ is expressed as:

$$
\Delta t_{i}=2 \cdot \operatorname{sgn}\left(z-z_{f}\right) \cdot \frac{\left(r^{\prime}-r\right)}{c}
$$




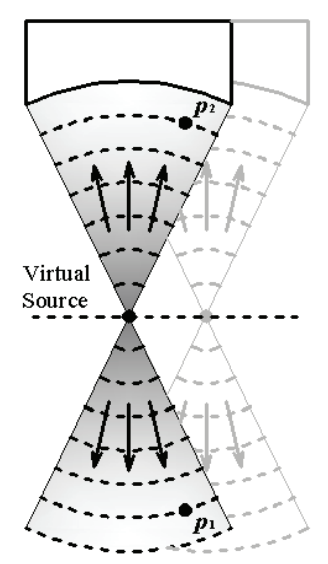

(a)

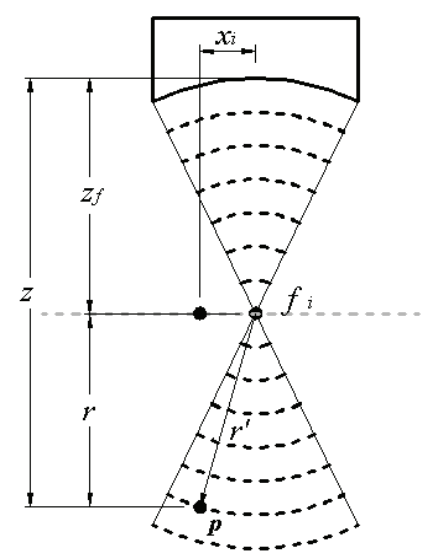

(b)

Figure 4.3 (a) Sketch for virtual-source concept. (b) Focusing geometry for the SAFT with a virtual source, where $z$ is the depth of the synthetic focal point $p, z_{f}$ is the transducer's focal depth, $r$ is the axial distance from the transducer's focal depth to the synthetic focal depth, $r^{\prime}$ is the distance from the virtual source $f_{i}$ to the synthetic focal point $p$ [88].

where $z$ is the depth of the synthetic focal point $p, z_{f}$ the transducer's focal depth, $r=\operatorname{abs}\left(z-z_{f}\right)$ the axial distance from the transducer's focal depth to the synthetic focal depth, $r^{\prime}=\left(r^{2}+x_{i}^{2}\right)$ the distance from the virtual source $f_{i}$ to the synthetic focal point, $c$ the speed of sound, and $\operatorname{sgn}(\cdot)$ the signum function [88]. Note that, if the synthetic focal point is located in front of the transducer's focal point, the time delay is negative. After appropriate delays relative to the virtual source are applied to all corresponding scan lines according to the above equation, weighted SAFT is implemented as the following sum:

$$
A(t)=\sum_{i} w_{i} S_{i}\left(t-\Delta t_{i}\right)
$$

where $A(t)$ is the computed RF echo return, and $w_{i}$ is a weight assigned to the returned signal, $S(t)$, for scan line $i$. The number of signals included in the sum is determined by the aperture angle of the transducer beam beyond the transducer's focus. The 
beam spread of a source is determined by the geometry of the source and the wavelength of sound in the medium [87]. Both virtual and aperture focal points are adaptively changing based on the field of view (FOV) or the region of interest (ROI). The upper part of the FOV/ROI is placed near the transducer virtual focus, where the aperture focus is kept at the bottom.

In this work, apodization weights, or windows in signal processing, are applied in receive mode. Signal processing theory shows that the windowing operation broadens the main lobe and lowers the sidelobes [89]. Several windows have been tested with SAFT to improve the spatial resolution and minimize the effects of the sidelobes. Boxcar, triangle, cosine, and hamming windows have been tested. After applying SAFT, a signal processing algorithm is applied to extract the signal envelop by calculating the amplitude of the Hilbert transform of the echo signal. Logarithmic compression is then applied to reduce the dynamic range for visualization. A simple function is implemented for compression and to keep most of the image details. At this stage, the B-mode image is obtained but image enhancement techniques could be applied to reveal small details and to improve the image contrast.

\subsubsection{Image Processing and Resolution Improvement}

A phase-preserving algorithm based on decomposing the signal using complexvalued wavelets is employed to improve the image quality and decrease the noise [90]. This algorithm utilizes non-orthogonal, complex valued, log-Gabor wavelets for converting the image to the transform domain. In this technique, the appropriate wavelet shrinkage thresholds are automatically determined from the statistics of the amplitude response of the smallest scale wavelet quadrature pair. Transforms are then clipped at the threshold and the inverse transform is taken to get the improved image. It is well known that the use of single-element ultrasound transducer, particularly high-frequency, usually incorporates resolution degradation within the images [87,88], [91]. This degradation is mainly caused by the convolution of a virtual scanning point spread function (PSF) that is a function of both ultrasound transducer and depth. This degradation is partially reduced by applying weighted SAFT, however, applying image-based blind deconvolution would add further improvement. For this purpose, we have incorporated a deconvolution technique based on using the maximum likelihood algorithm [92]. In this technique, Lucy-Richardson procedure 
was used to restore the image that was degraded by convolving with a PSF. Estimates of the point spread function were determined at different locations using a custom designed 5-pin wire phantom. Finally, the effect of speckle noise was reduced using the Perona-Malik algorithm. The noise manifested as dark lines elongated in the lateral direction. Since the Perona-Malik algorithm encourages smoothing within homogeneous regions and discourages smoothing between homogeneous regions the resulting images show better edge contrast than those obtained using Gaussian convolution [93]. This allows regions of cardiac muscle to be more clearly distinguished from, for example, the ventricles. Finally, images are linearly mapped to gray scale levels for display at the proper dynamic range such as $80 \mathrm{~dB}$.

\subsubsection{Phantom Study}

Different studies using wire phantoms are performed for evaluating the system performance and its focusing ability at different depths. Two configurations of wires are used with different wire diameters; single steel wire $(25 \mu \mathrm{m})$ and multi-wire tungsten phantom $(40 \mu \mathrm{m})$. The single steel wire was used to evaluate the system focusing strength with the use of the adaptive weighted SAFT technique. The wire is placed at about $3 \mathrm{~mm}$ beyond the transducer focal point. Five-pin configuration was constructed in our shop with specific geometry shown in figure 4.7(a). The wires are separated in the lateral and axial directions by $2 \mathrm{~mm}$ to demonstrate the system axial and lateral resolution. In each experiment, both of the phantom and the transducer were immersed in degassed water that acts as the coupling media. The phantom is placed such that the top wire located near the transducer focus and the aperture virtual focus is selected near the lowest wire. Two sets of RF signals were collected from the phantoms, one for each phantom, with a separation distance around $13 \mu \mathrm{m}$ between any two adjacent received signals.

\subsubsection{In Vitro Study}

In vitro experiments are conducted to investigate the efficacy of the system on reconstructing ultrahigh resolution B-mode images for real objects. Studies are performed to image isolated mouse hearts of APOE-KO model (20-34 week old male with atherosclerotic heart) and its wild type control (C57BL/6J, an 8 week old male mouse with normal heart). Mice are taken from the colony of Dr. Mustafa in the 
Health Science Center (HSC), West Virginia University (WVU), Morgantown, WV. The animal care and use program at the HSC is accredited by the Association for Assessment and Accreditation of Laboratory Animal Care International (AAALAC). All mice procedures are performed and monitored by professionals in the field of cardiovascular research and according to the protocols approved by the WVU Animal Care and Use committee. To prepare the isolated heart, mice are euthanized by deep anesthesia using pentobarbital sodium $(100 \mathrm{mg} / \mathrm{kg}$ ip). Then, a thoracotomy is performed and the heart is removed. Hearts are preserved in the oxygenated high potassium buffer (St. Thomas Solution) to stop spontaneous heart beat and keep them fresh for the ultrasound imaging procedures. Objects are placed near the transducer focus and RF signals are collected from the FOV using separation distances down to $11 \mu \mathrm{m}$ between adjacent signals. Virtual aperture focal points are kept near the bottom of different objects in order to retain relatively good resolution at different depth.

\subsection{Results and Discussion}

Typical B-mode images for the $25 \mu \mathrm{m}$ in diameter steel wire reconstructed using different weighting windows are shown in figure 4.4. These images are acquired using the $100 \mathrm{MHz}$ ultrasound transducer using a dynamic range of $80 \mathrm{~dB}$. The resolution improvement, when using SAFT, is observed in both axial and lateral directions when comparing the raw image shown in figure 4.4(a) with other images. These images are reconstructed after applying SAFT using different weighting windows. It is difficult to detect which window performs better using only B-mode images. So the effect of each weighting window when applying SAFT is analyzed by calculating the 1D point spread function (PSF) for each case. Figure 4.5 describes both the axial and the lateral PSF with different windows. It is observed that the boxcar window produces images with the best lateral resolution for all depth. However, the sidelobes are also the highest for all the depth compared with other windows but at low $\mathrm{dB}$. We also found that the cosine window balances between the lateral resolutions and sidelobes, which provides an acceptable solution. These results of the windowing effect described here match with the ones in original SAFT study [87]. However, the 


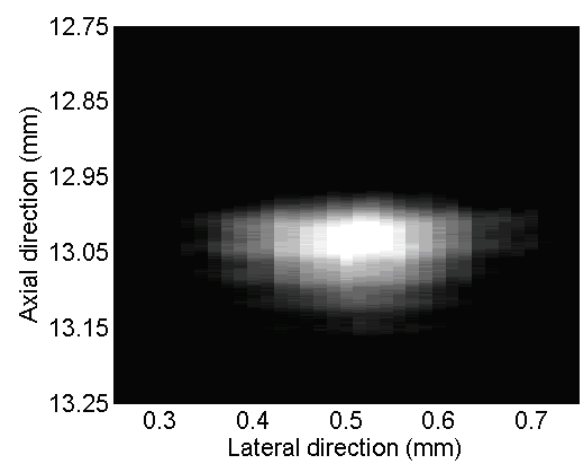

(a)

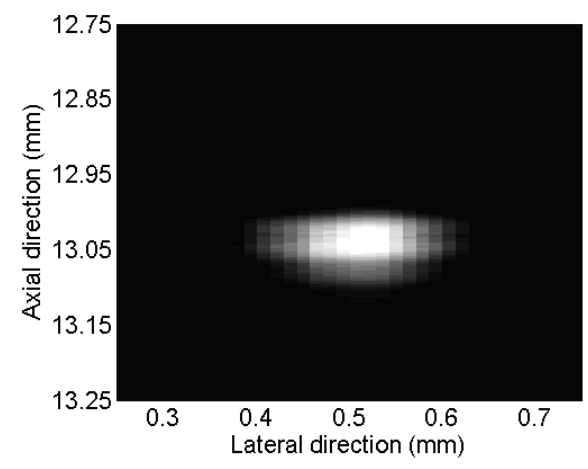

(c)

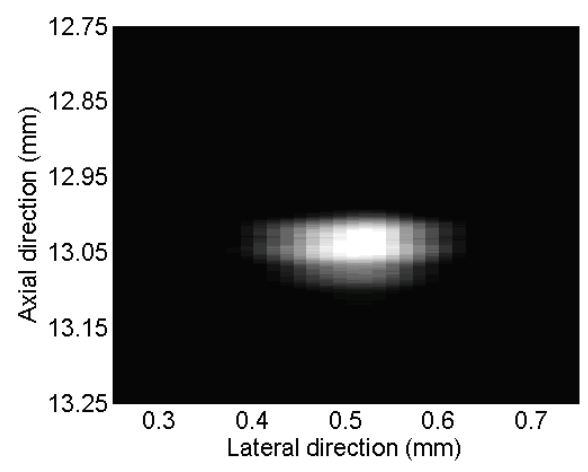

(b)

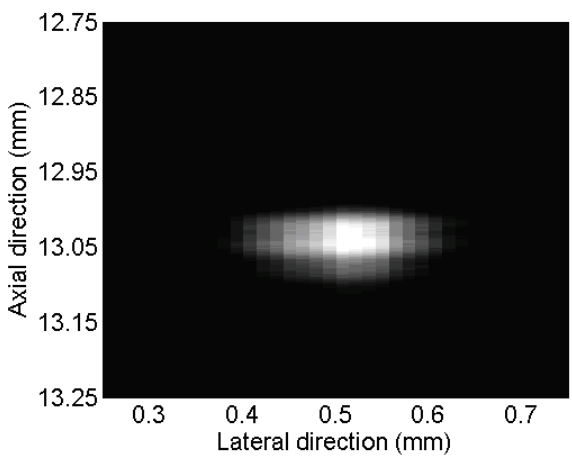

(d)

Figure 4.4 B-mode images for steel wire phantom of $25 \mu \mathrm{m}$ of diameter immerged in degassed water acquired using $100 \mathrm{MHz}$ transducer. Images reconstructed using the synthetic aperture focusing technique. The wire is placed $3 \mathrm{~mm}$ beyond the focus. Different weighting windows are used: (a) Raw Image, (b) Image reconstructed using SAFT with Hamming window, (c) cosine window, and (d) boxcar.

resolution improvements are considered better here due to the higher frequency used and different acquisition technique used.

Based on the system specification, this ultrasound system can achieve theoretical axial and lateral resolution of 10.3 and $51.4 \mu \mathrm{m}$ respectively using Eqs. (4.3) and (4.4) [55] described below:

$$
R_{\text {lat }}=\lambda * \frac{F D}{A}
$$




$$
R_{a x}=\frac{1}{2} * \frac{c}{B W}
$$

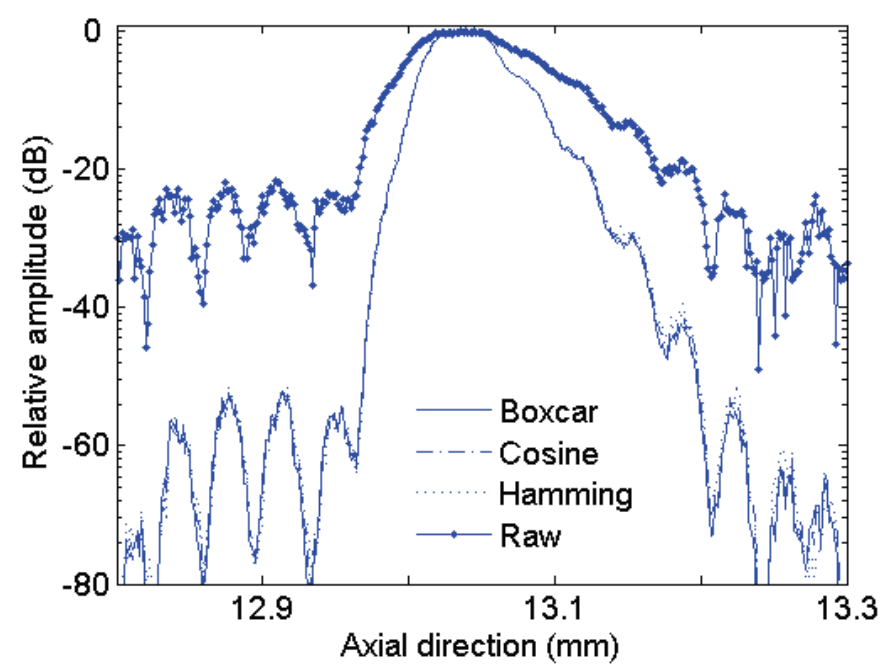

(a)

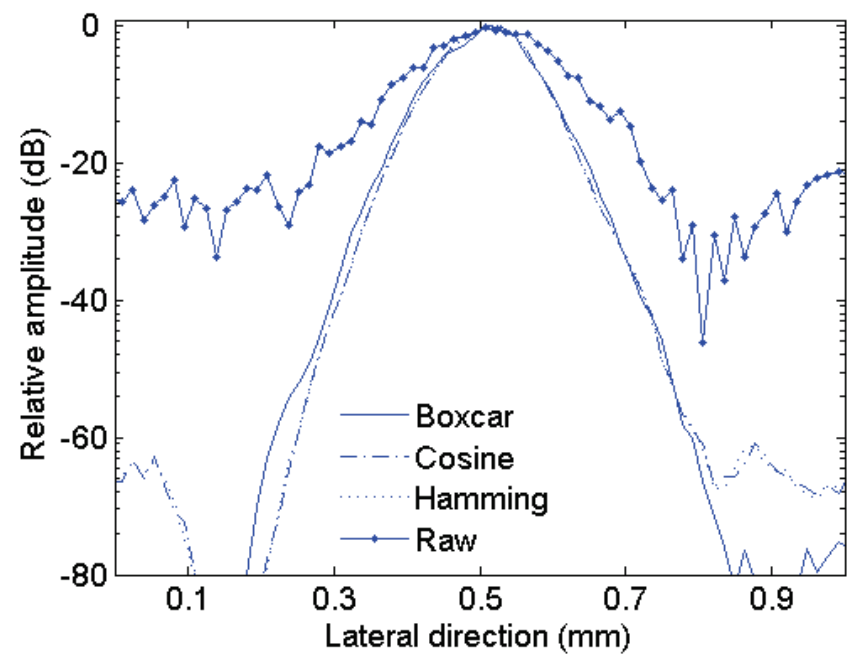

(b)

Figure 4.5 Axial and lateral beam plot for the $25 \mu \mathrm{m}$ steel wire using the $100 \mathrm{MHz}$ transducer. Plots show the change in the beam spreading with different weighting windows. Boxcar, cosine, and Hamming windows are used. (a) Axial plot, and (b) lateral plot. 
where $\lambda$ is the wavelength at the center frequency calculated using a speed of 1540 $\mathrm{m} / \mathrm{s}, F D$ the focal distance, $A$ the transducer diameter, $c$ the speed of sound, and $B W$ the transducer bandwidth. The experimental values for both axial and lateral resolutions are measured using analogous method described in [91].These values are found after applying weighted SAFT to be 41 and $71 \mu \mathrm{m}$ for the axial and lateral resolutions respectively at $-6 \mathrm{~dB}$. In the lateral direction, the measured resolution has a better match with the theoretical value than the axial resolution with an accepted deviation. One major cause of the difference is the small spatial shift of the wire out of the transducer focus, $3 \mathrm{~mm}$ in this experiment, which causes the wire elongation. Axially, there is a great difference between the measured and calculated axial resolution. Although this effect could be reasonable and was expected due to the 25 $\mu \mathrm{m}$ wire diameter, further resolution improvements were obtained after applying the image-based deconvolution. Figure 4-6 demonstrates how the 2D PSF was improved after applying the deconvolution technique, when comparing with the PSF of the raw image (figure 4-6(a)) and the one after applying weighted SAFT (figure 4-6(b)). Both axial and lateral resolutions were evaluated experimentally at $-6 \mathrm{~dB}$ using the 1D PSF (figure 4-6(d)) of the final image. This system is able to achieve axial and lateral resolutions of 18 and $57 \mu \mathrm{m}$ respectively.

In spite of the excellent resolution of high-frequency ultrasound systems provide near the transducer focus, the overall image spatial resolution degraded drastically for wires out of the transducer focus. Therefore, adaptive image reconstruction techniques are applied to overcome this problem. SAFT with virtual focus is used where the top of the ROI is placed at the transducer focus, and the virtual focus is adaptively changing according to the bottom of the ROI. A boxcar weighting window is used during focusing. To assess the focusing ability of the system at different depths, different B-mode images for the five-pin phantom described earlier are reconstructed with multiple transducers. Figure 4.7(a) shows the phantom schematic, while figure 4.7(b) describes a typical B-mode image reconstructed for the wire phantom when applying adaptive SAFT. The B-mode image shows good agreement with the phantom with less change in the resolution at different depth than the phantom image (figure 4.7(c)) shown in Sun et al. [91]. Using this system with the adaptive weighted SAFT, allowed us to overcome the problem of vast resolution degradation that usually occurs during the multiple wire 
(a)

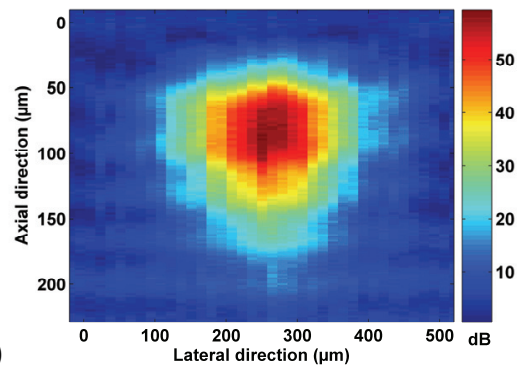

(c)

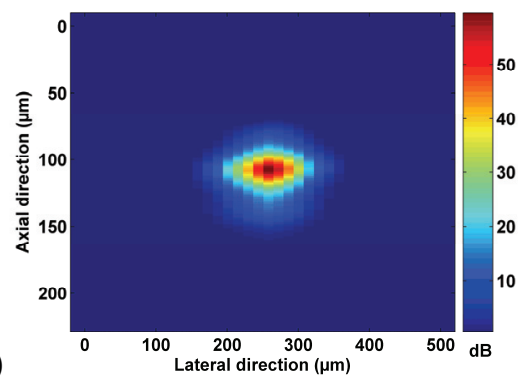

(b)

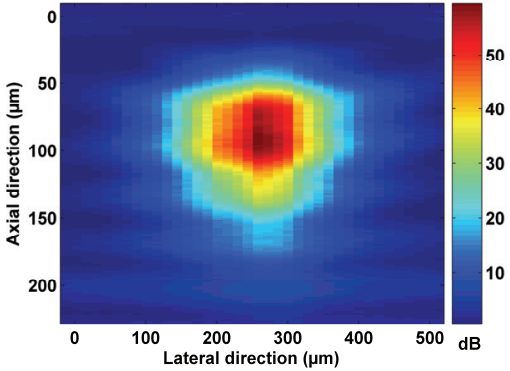

(d)
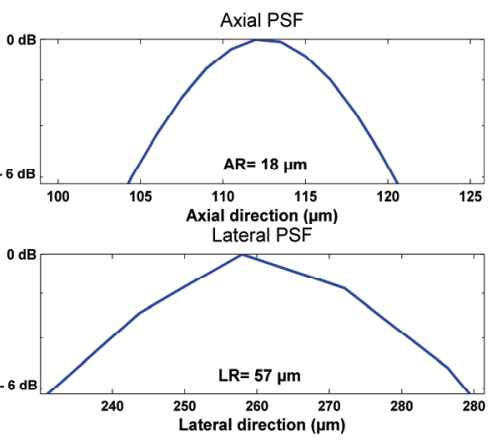

Figure 4.6 Spatial resolution enhancement shown using 2D point spread function (PSF). A bird's eye view of a) raw image, b) image after applying weighted SAFT, and c) image reconstructed after applying 2D PSF deconvolution technique, and d) axial and lateral PSF plots showing axial and lateral resolutions of 18 and $57 \mu \mathrm{m}$, respectively.

tests [91]. Dynamic deconvolution was also applied to the phantom image (figure 4.7(b)) using estimated point spread functions at different depths. Further improvements in both directions were obviously observed in the phantom image at all depths where the resolution was improved at all depths. Significant improvement is clearly observed when comparing the wire image after applying 2D PSF deconvolution techniques (Fig. 4.7(d)) with other phantom images. In this work, we overcame the problem of spatial resolution degradation and we observed that the lateral resolution is almost homogenous in all depths with small changes. This problem of resolution degradation with depth has been described recently by Sun et al [92], in particular when target wires are shifted from the transducer focus. This effect may be ignored when the ROI is small $(<2 \mathrm{~mm})$ and centered around the transducer 


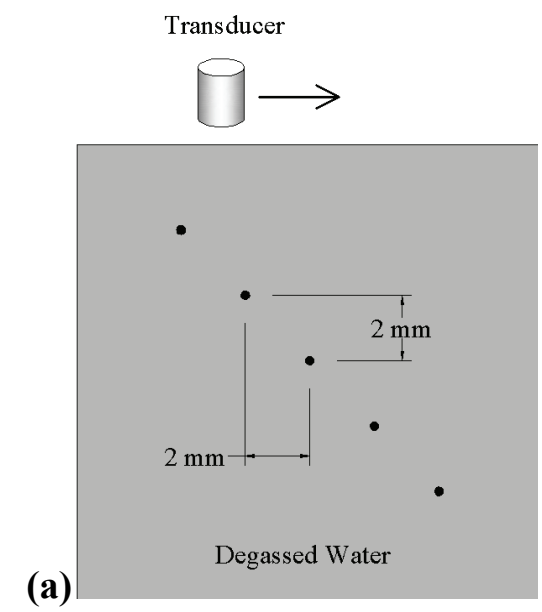

(b)

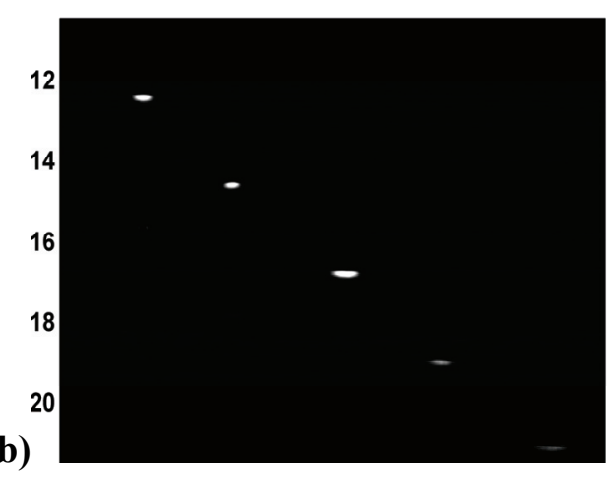

(c)

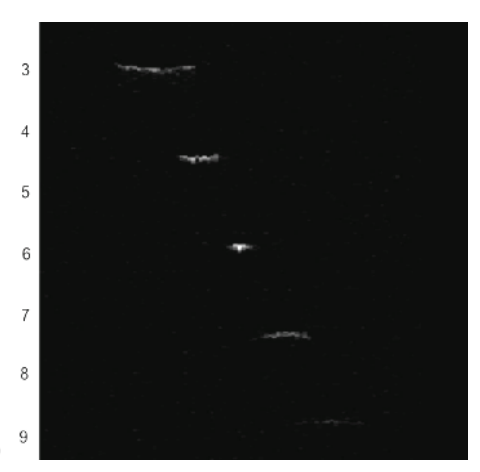

(d)

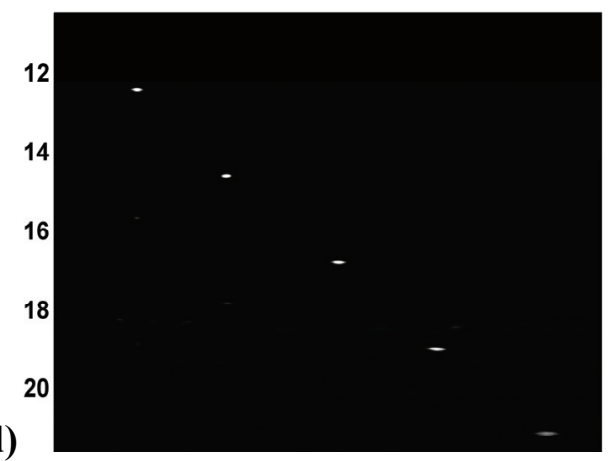

Figure 4.7 (a) Schematic of wire-phantom, (b) B-mode image of the wire phantom using our system with adaptive weighted SAFT only (c) B-mode image of a wire phantom [91], and (d). B-mode image for the wire phantom using both adaptive SAFT and dynamic deconvolution. The use of both techniques overcomes the problem of spatial resolution degradation, shown in (c), where the lateral resolution is almost homogenous at different depths.

focus, but here the system is designed to cover a wide (up to $250 \mathrm{~mm}$ ) and deep (up to $30 \mathrm{~mm}$ ) field of view.

It is important after testing the system efficacy and resolution using wire phantoms to test the system using real objects. Various experiments have been performed to image cardiovascular tissues of small animals in vitro. The system efficacy and final Bmode image quality are tested via acquiring multiple images for isolated mouse hearts and blood vessels for normal and genetically-modified mouse models. The reason behind performing in vitro studies using mouse hearts as tissue samples is to develop 
essential testing of our system for future in vivo animal imaging utilizing high framerate imaging capability. Mouse hearts preparation and preservation steps have been previously described (section 4.1.5). Figure 4.8 shows photographic images for a typical mouse heart relative to a metric ruler. That heart has long and short axis lengths of about 8 and $5 \mathrm{~mm}$, respectively, which demonstrates how challenging the imaging process is. The ability of the advanced image processing procedures applied to improve the image quality and contrast after the logarithmic compression stage is demonstrated in figure 4.9. Figure 4.9(a) shows an initial B-mode image, after logarithmic compression, for a long axis scanning of 8 -weeks old male C57BL/6J mouse heart. The image has low contrast and low signal to noise ratio except at boundary regions of the aorta due to strong reflections. After applying the advanced image processing procedure, even without deconvolution, the image quality has been enhanced and different structures within the heart become visible and distinguishable. Among these structures are the right atrium (RA), right ventricle (RV), left atrium (LA), left ventricle (LV), and the aorta (AO). All heart structures shown in this thesis were assigned based on the review of professionals in the field of cardiovascular research and mouse anatomy, in addition to comparisons with mouse atlas.

(a)

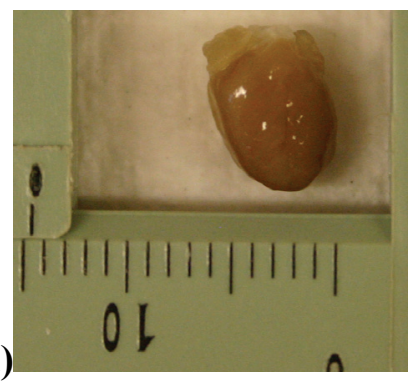

(b)

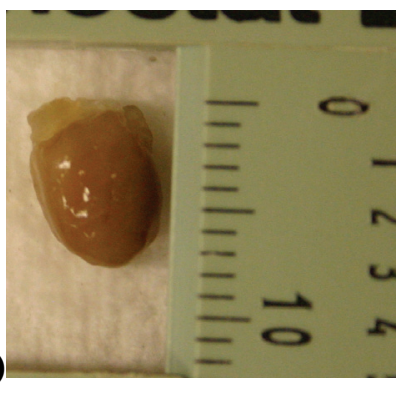

Figure 4.8 Photographic images describe the size in mm for a typical mouse heart. (a) Short axis, and (b) long axis.

Short axis scanning of 8 -weeks old male C57BL/6J mouse heart is performed 1 mm apart. Four B-mode images for different slices of the short axis are shown in figure 4.10. Slices are acquired starting from the lower region near the apex and upward towards the atria. Different structures are visible, such as, the LV, RV, RA, 


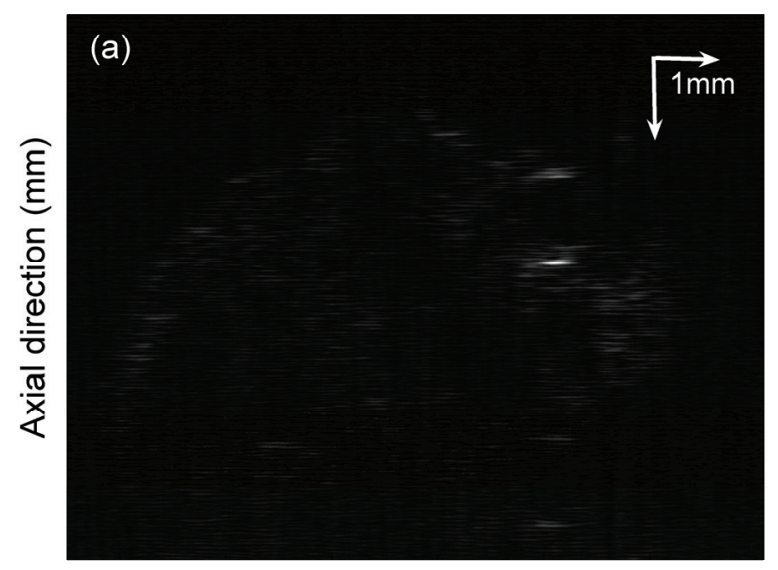

Lateral direction $(\mathrm{mm})$

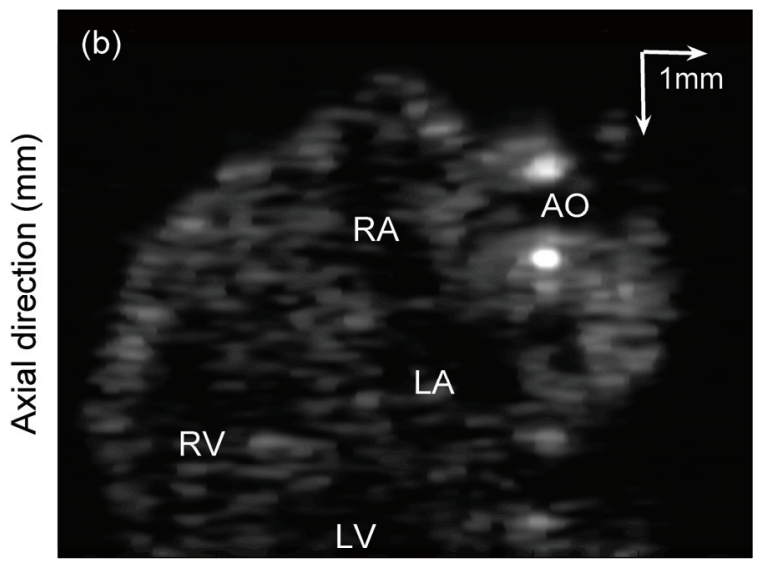

Lateral direction $(\mathrm{mm})$

Figure 4.9 Images of the long axis of 8-weeks mouse heart. (a) Image after logarithmic compression stage, and (b) image after advanced image processing stage. RV, right ventricle. LV, left ventricle. RA, right atrium. AO, Aorta. LA, Left Atrium.

left ventricle (LV), and the septum that separates the two ventricles. The boundaries and contours of each structure are well defined which enables accurate qualitative and quantitative morphological assessments. Also, multiple longitudinal scans are performed for the same mouse heart which reveals more details. Figure 4.11 describes two B-mode images for the longitudinal axis of the mouse heart acquired 1 mm apart. In addition to the RV, LV, RA, and LA that are shown, more structure can be seen and be differentiated like the pulmonary artery (PA), and the tricuspid valve (TV). 


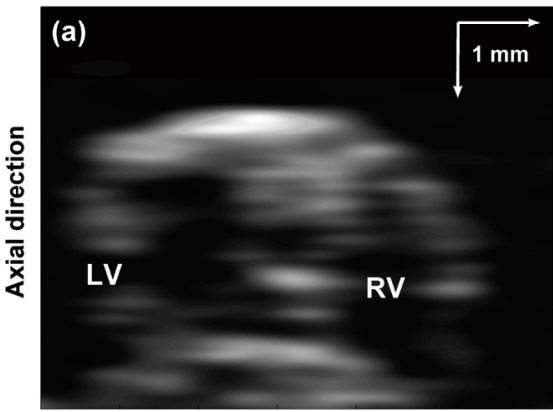

Lateral direction

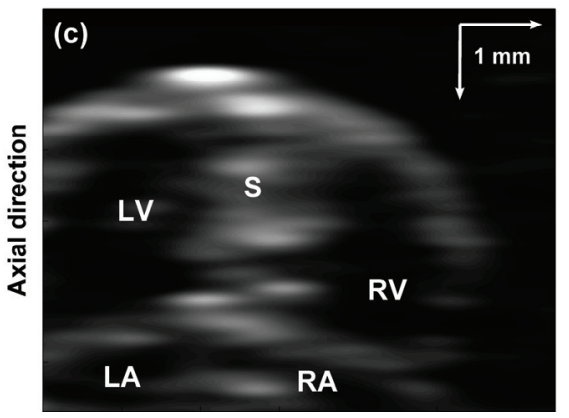

Lateral direction

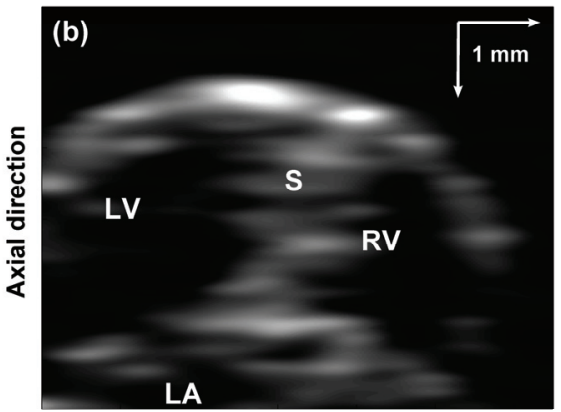

Lateral direction

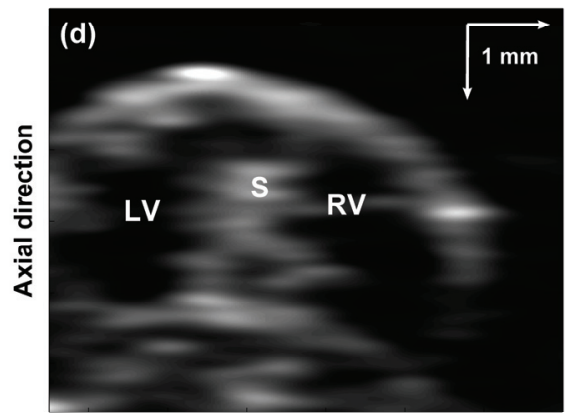

Lateral direction

Figure 4.10 Final B-mode images of the short axis of 8-weeks mouse heart. Different slices acquired $1 \mathrm{~mm}$ apart. $\mathrm{RV}$, right ventricle. $\mathrm{LV}$, left ventricle. RA, right atrium. LA, left atrium. S, septum.

Further in vitro studies were performed for demonstrating the precision and the repeatability of the scanning process of our high-frequency ultrasound system. Multiple scans were acquired for a small region within an isolated mouse heart of 20weeks male APOE-KO mouse. Figure 4.12 describes three B-mode images of the short axis view of the mouse heart acquired $1 \mathrm{~mm}$ apart. Different structures can be recognized including the $\mathrm{RV}, \mathrm{LV}$, and the mitral valve (MV), and can be tracked through the slices. For the same region, three scans $0.5 \mathrm{~mm}$ apart were acquired for the long axis view. Same structures were detected, in addition to the aortic root (AR), and LA (see figure 4.13). This correlation, demonstrated here, between different structures within the long and short access views emphasis the system precision and repeatability. Moreover, these properties will provide cardiovascular researchers, noninvasively, with slices for both the long and short axis views within same small regions which may not be possible when using manual cutting. 


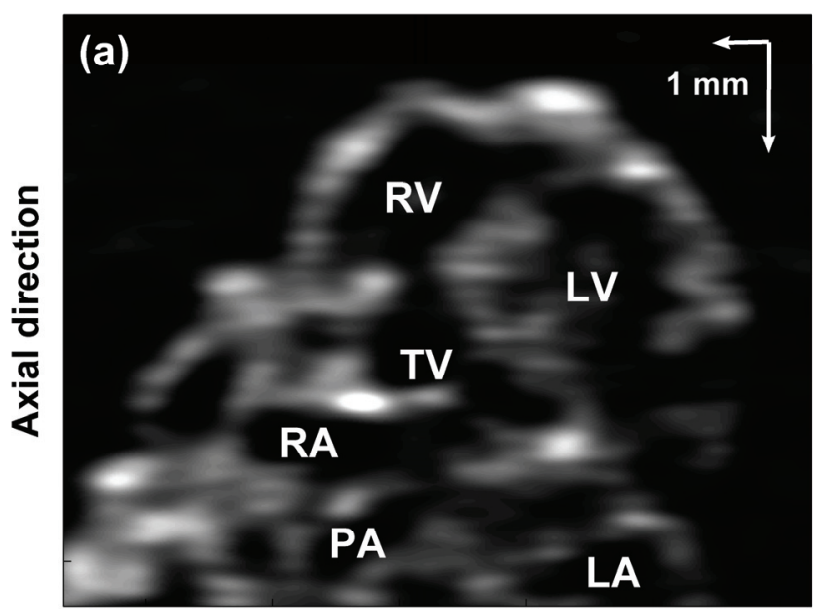

Lateral direction

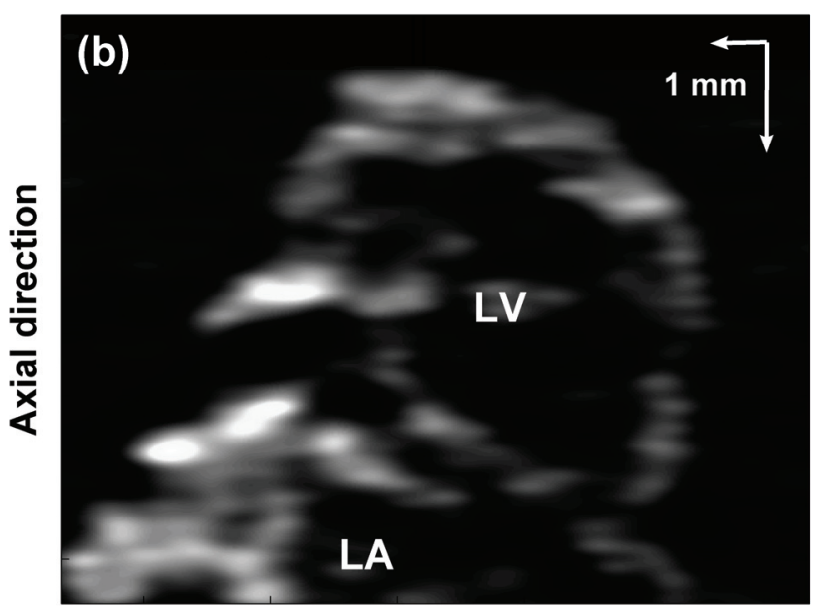

Lateral direction

Figure 4.11 Images of the long axis of 8-weeks mouse heart. Different slices acquired $1 \mathrm{~mm}$ apart. RV, right ventricle. LV, left ventricle. RA, right atrium. LA, left atrium. $\mathrm{TV}$, tricuspid valve. PA, pulmonary artery.

Comparison between B-mode images and optical images of the heart structure reveals remarkable similarity between acquired images and real objects. This comparison is demonstrated in figure 4.14 by comparing the B-mode image of heart cross-section and a photographed image of the same heart near the cross-section scanned. The photographed image was taken after slicing using a digital camera fixed on the tip of high magnification optical microscope. Isolated mouse heart of 34weeks male APOE-KO mouse with atherosclerosis has been used in this study. Fibrous atheroma accumulations are shown in both images inside the circle which 
block the aortic sinus. Moreover, noticeable matching between the structures in both images is observed.

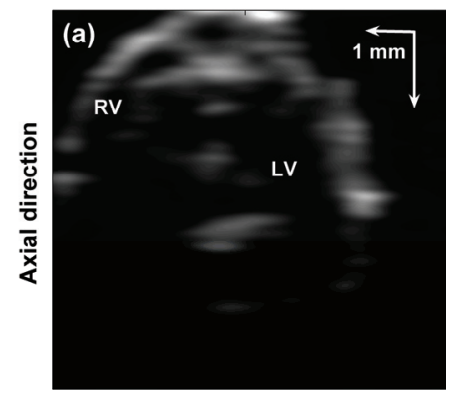

Lateral direction

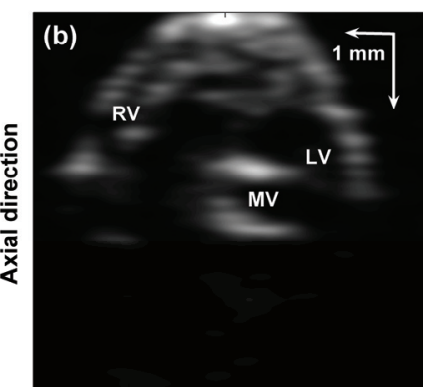

Lateral direction

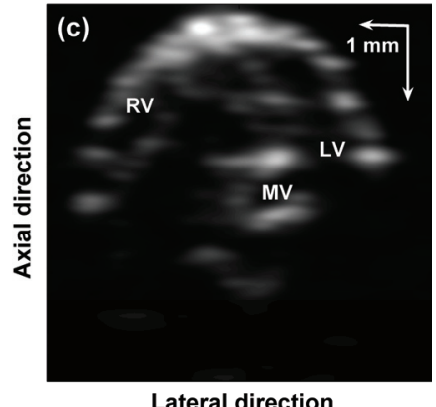

Lateral direction

Figure 4.12 B-mode ultrasound images for the short axis view of isolated mouse heart of 20-weeks APOE-HFD-Male. Different slices acquired $1 \mathrm{~mm}$ apart. RV: right ventricle. LV: left ventricle; MV: mitral valve.

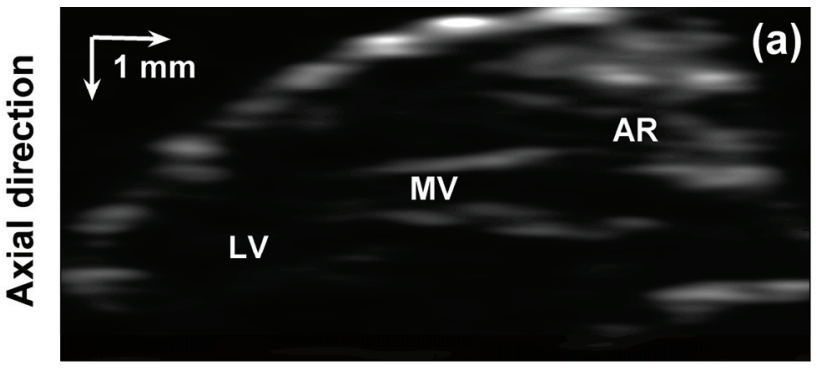

\section{Lateral direction}

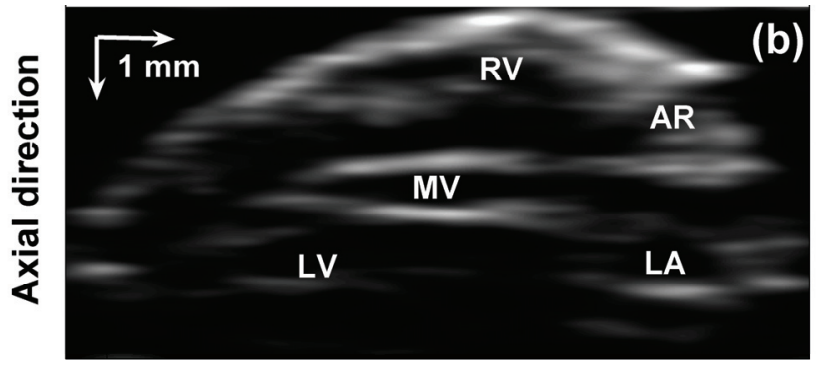

Lateral direction 


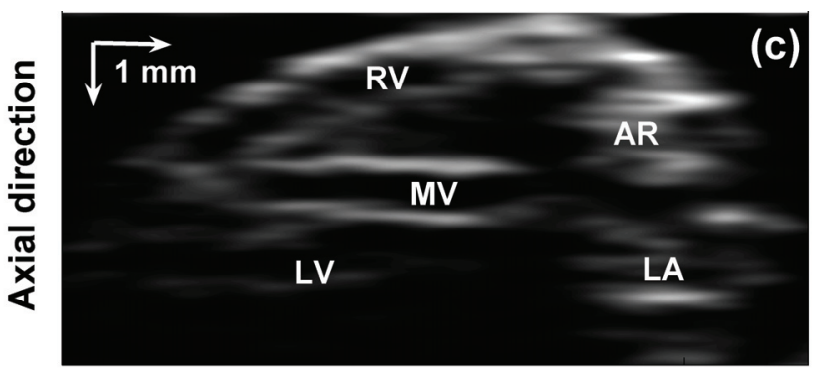

Lateral direction

Figure 4.13 B-mode ultrasound images for the long axis view of isolated mouse heart of 20-weeks APOE-HFD-Male. Different slices acquired $0.5 \mathrm{~mm}$ apart. RV: right ventricle. LV: left ventricle; LA: left atrium; MV: mitral valve; AR: aortic root.
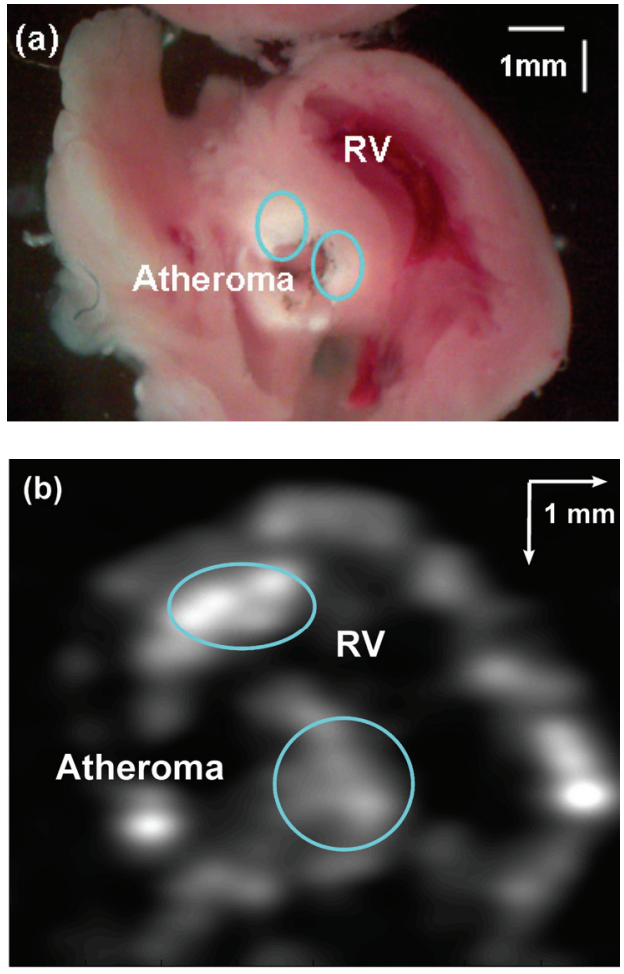

Figure 4.14 APOE-HFD- Male-34 week-cross section. a) Image acquired using a camera on the tip high magnification microscope. b) Image acquired using our system ex vivo near the cross section in (a) before cut. RV: right ventricle. 


\section{CHAPTER 5}

\section{in Vivo VAScular Wall Tissue Characterization USIng A STRain Tensor Measuring (STM) TECHNIQUE FOR FLOW-MEDIATED VASODILATATION ANALYSES}

Endothelial dysfunction is considered to be a key factor in the development of atherosclerosis, and the measurement of flow-mediated vasodilation (FMD) in brachial and other conduit arteries has become a common method to asses the status of endothelial function in vivo. In spite of the strong relationship between the arterial wall local shear stresses and FMD responses, direct measurement of wall strain tensor due to FMD has not yet been reported in the literature. In this work, a noninvasive direct ultrasound-based strain tensor measuring (STM) technique is presented to assess changes in the mechanical parameters of the vascular wall during FMD, including local velocities and displacements, diameter change, local strain tensor and strain rates. The STM technique utilizes sequences of B-mode ultrasound images as its input with no extra hardware requirement. The accuracy of the STM algorithm is examined using phantom studies using different displacement scenarios. Furthermore, in vivo studies using human subjects are performed to test the STM algorithm during pre- and post-occlusion, and to investigate how parameters other than the diameter change are sensitive to pre- and post-occlusion. In the following sections, the STM algorithm methods are explained followed by the results and discussion section.

\subsection{Methods}

\subsubsection{Displacement and Strain Measurements}

The schematic diagram, shown in figure 5.1, illustrates an overview of the STM algorithm procedures for measuring the brachial artery mechanical properties using ultrasound imaging during pre- and post-occlusion intervals. This STM technique 
measures tissue displacement components within regions of interests (ROI) in Bmode ultrasound images by measuring the relative displacements between consecutive frames. As a first step, the ultrasound transducer is aligned parallel to the brachial artery and a sequence of B-mode images is acquired for the brachial longitudinal view as shown in figure 5.2. The schematic diagram shown in figure 5.2(a) describes the expected field of view and explains how the transducer is aligned

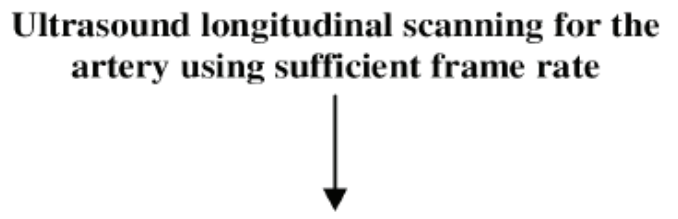

Saving the B-mode images sequence, then manual segmentation for ROIs

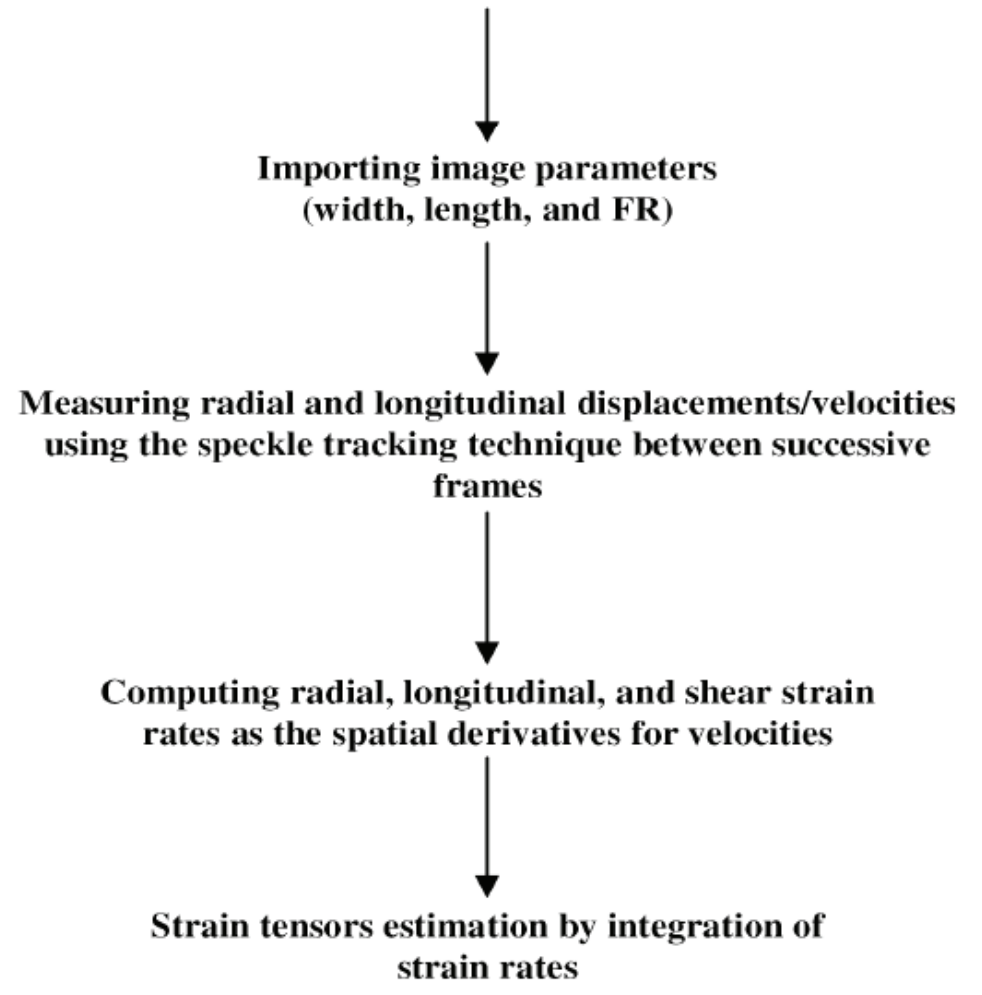

Figure 5.1 Schematic overview of the algorithm procedures required for measuring the arterial wall mechanical properties. Using the measured local velocity components, other parameters can be calculated by further integration or differentiation steps. 
with the artery direction. Further, figure 5.2(b) shows B-mode ultrasound image for the right brachial artery of healthy subject after logarithmic compression. ROI are then segmented manually for both sides of the arterial wall, and these ROI start from the lumen-intima interface and progress to the end of adventitia layer for both sides of the artery. After initialization, the STM technique measures the displacements/strains automatically.

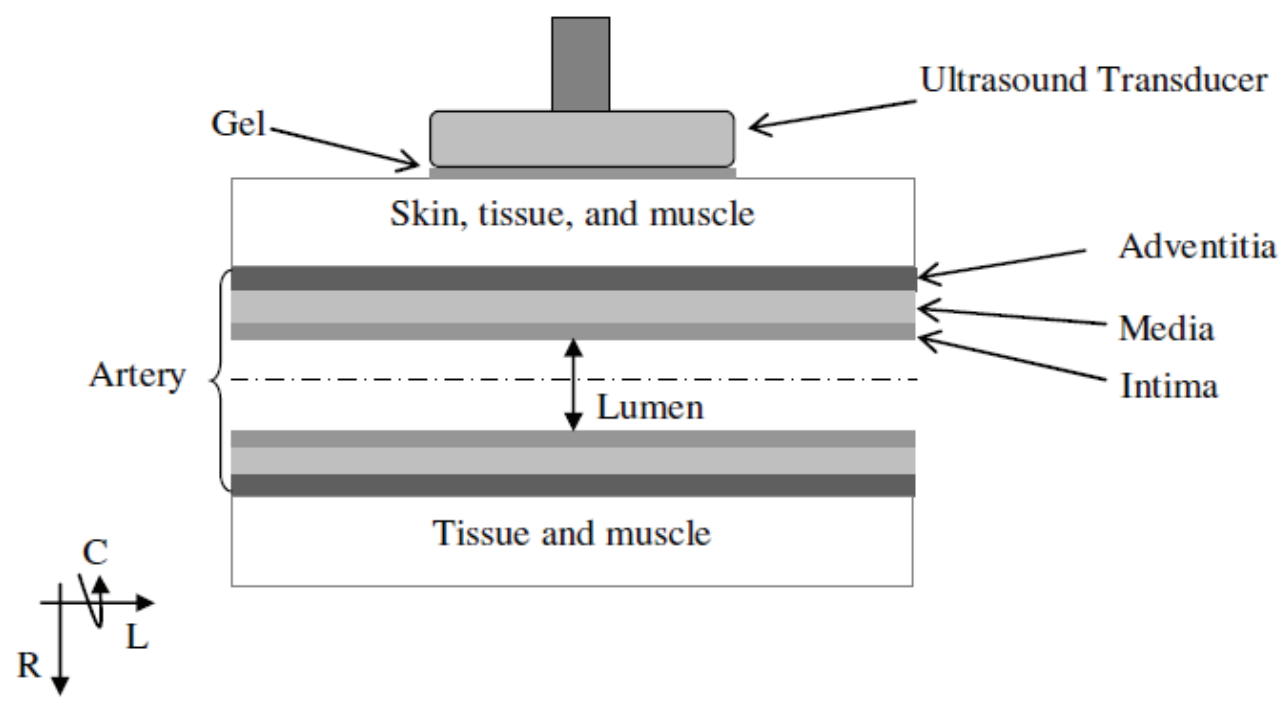

(a)

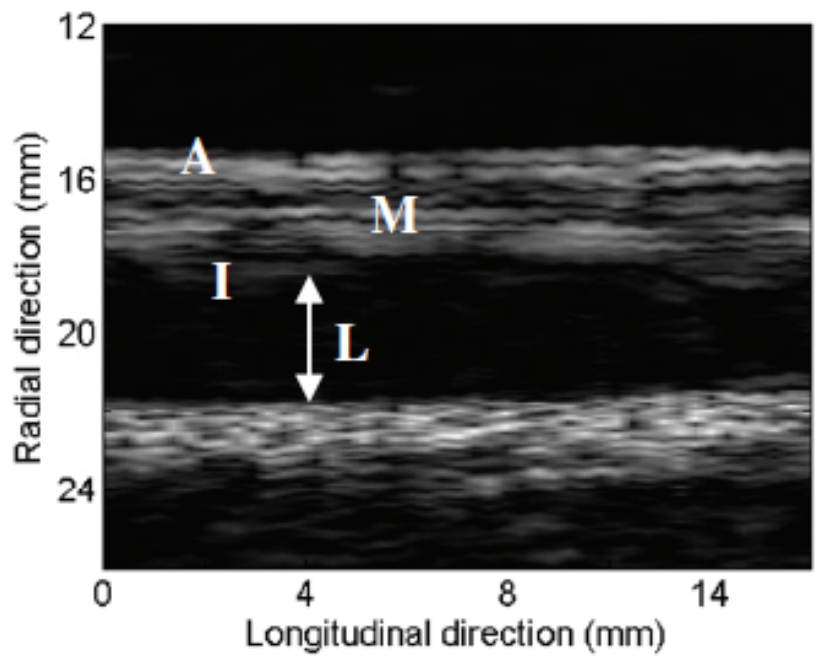

(b)

Figure 5.2 Brachial artery longitudinal views. (a) Schematic showing the ultrasound transducer alignment relative to the field of view, and (b) B-mode ultrasound image for the brachial longitudinal view. 
In order to measure tissue displacements using ultrasound images, 2-D speckle tracking techniques are commonly used [94-97]. The speckle tracking, based on minimizing the sum of absolute difference (SAD) method [95],[98], is used to estimate tissue motion in both axial and lateral dimensions within the arterial walls (i.e., near and far walls). The use of SAD as the objective function overcomes the problem of computational complexity of the correlation algorithms, which facilitates the real time implementation [94]. Moreover, both the SAD and normalized correlation algorithms showed similar performance when different kernel sizes and noise levels were used [99]. In this technique, the first ultrasound frame (B-mode image) is divided into two-dimensional "kernel" regions. Consequently, the next frame, which is acquired at a later time for a two-dimensional region, includes and surrounds the identical kernel region. Equation (5.1) is evaluated for each value of $\alpha$ and $\beta$.

$$
S A D(\alpha, \beta)=\sum_{i=1}^{m} \sum_{j=1}^{n}|k(i, j)-s(i-\alpha, j-\beta)|,
$$

where $\operatorname{SAD}(\alpha, \beta)$ is the sum of absolute difference between the kernel and search for the displacement components $(\alpha, \beta), k(i, j)$ is the kernel region value at $(i, j)$, and $s(i$, $j$ ) is the search region value at $(i, j)$, and $m$ and $n$ are the width and length of the kernel in pixels. A best match occurs where $\operatorname{SAD}(\alpha, \beta)$ falls into a minimum. Therefore, the location of the best match of each kernel indicates the tissue movement between the two successive frames. Figure 5.3 describes this scenario using an image of one of the arterial walls in which the solid rectangle represents the kernel region while the dashed represents the search region. Kernel sizes of $64 \times 64$ pixels down to $16 \times 16$ pixels with various overlapping ratios 0 up to $90 \%$ can be used. In this work, kernels of $32 \times 32$ pixels $(\approx 2.5 \times 2.5 \mathrm{~mm})$ in dimension are used using $50 \%$ overlapping ratio. These values maintain the kernel condition suggested by Bohs and Trahey [94], and compromise between the processing time, and the minor accuracy improvement that can be achieved by a further decrease in the kernel size or increase in the overlapping ratio at this resolution. The two-dimensional displacement components are displayed as an arrow, which indicates the magnitude and the 


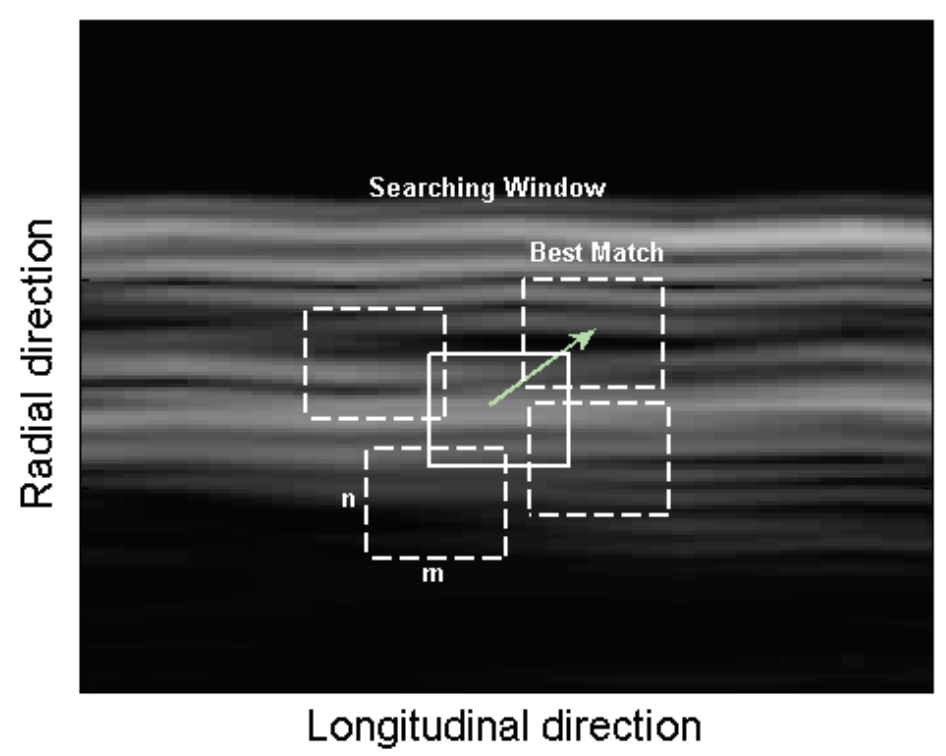

Figure 5.3 The Speckle tracking technique is demonstrated using a B-mode ultrasound image for one of the arterial walls.

direction of the tissue motion, overlapped on the B-mode image. Frame-to-frame values of the axial and lateral displacement components for each sub-window within the B-mode image are saved in vectors with the corresponding time. Using the acquisition frame rate, both the axial and lateral velocities ( $u$ and $v$, respectively) are calculated by dividing the axial and lateral displacement components by the temporal resolution. A custom Matlab toolbox, analogous to the 'MPIV' toolbox [100], is used to compute the velocity components. After calculating velocity vectors, post processing procedures are applied to eliminate spurious vectors, which are considered intrinsic to the block matching based speckle tracking method, and to improve the overall accuracy. In the post processing, a median filter is applied to each vector comparing it with its neighborhood of vectors (typically $3 \times 3$ or $5 \times 5$ ) within small areas to determine whether it is spurious or not. If the vector is considered spurious, Kriging interpolation is used to generate a substitute vector using the neighborhood of vectors.

Once the displacement components for each kernel are determined, diameter change can be calculated as the relative radial displacement change at the near and far walls as: 


$$
\Delta D=R_{w 2}-R_{w 1}
$$

where $\Delta D$ is the diameter change, and $R_{w 1}$ and $R_{w 2}$ are the radial displacements for kernels in the near and far wall boundaries, respectively.

The components of radial, longitudinal, and shear strain rates can be expressed as the spatial gradient for local velocity components [101]. In this work, these parameters are expressed as:

$$
\begin{gathered}
\varepsilon_{r}=\frac{v\left(x_{i}, y_{j+1}\right)-v\left(x_{i}, y_{j}\right)}{y_{i+1}-y_{i}}, \\
\varepsilon_{l}=\frac{u\left(x_{i+1}, y_{j}\right)-u\left(x_{i}, y_{j}\right)}{x_{i+1}-x_{i}}, \\
\gamma_{l r}=\frac{1}{2}\left(\frac{u\left(x_{i+1}, y_{j+1}\right)-u\left(x_{i}, y_{j}\right)}{y_{i+1}-y_{i}}+\frac{v\left(x_{i+1}, y_{j+1}\right)-v\left(x_{i}, y_{j}\right)}{x_{i+1}-x_{i}}\right),
\end{gathered}
$$

where, $\varepsilon_{r}, \varepsilon_{l}$, and $\gamma_{l r}$ are the radial, longitudinal, and shear strain rates, respectively. $v\left(x_{i}, y_{j+1}\right)$ and $v\left(x_{i}, y_{j}\right)$ represent the measured radial velocities in the radial direction of two kernels separated by a radial distance of $y_{i+1}-y_{i}$. Similarly, $u\left(x_{i+1}, y_{j}\right)$ and $u\left(x_{i}, y_{j}\right)$ are the measured longitudinal displacements of two kernels separated longitudinally by $x_{i+1}-x_{i}$ segment. Both $\left(x_{i+1}, y_{j+1}\right)$ and $\left(x_{i}, y_{j}\right)$ represent the locations of two kernels separated longitudinally by $x_{i+1}-x_{i}$, and in the radial direction by $y_{i+1}-y_{i}$. To calculate the local natural strain tensor (radial, longitudinal, and shear strains), temporal integration is applied to the calculated strain rates (i.e., Eqs. 5.3-5.5) as follows:

$$
\varepsilon_{r}=\int \varepsilon_{r} d t, \quad \varepsilon_{l}=\int \varepsilon_{l}^{\square} d t, \quad \text { and } \quad \gamma_{l r}=\int \gamma_{l r} d t
$$


Here, $\varepsilon_{r}, \varepsilon_{l}$ and $\gamma_{l r}$ are the radial, longitudinal, and shear strains, respectively, and $d t$ is the temporal resolution.

\subsubsection{Displacement Measurements: In Vitro Validation Study}

In order to test the ability of the STM algorithm to measure local displacements, various movement scenarios mimicking those of the vascular wall were applied to a wall phantom in both directions (radial and longitudinal). A speckle generating phantom with irregular boundary was used to mimic the moving target (vascular wall). This phantom consisted of a fine-grained sponge (approximately 5 cells $/ \mathrm{mm}$ ), and has been commonly used for displacement measurements validation using ultrasound images [94], [102]. Trahey et al. [103] have shown the ability of such target to generate ultrasound images that closely approximate random scatterer. A PC controlled two-axis (two translational) high precision positioning system with $1 \mu \mathrm{m}$ resolution (Danaher Corp., Washington, DC, USA) was used to apply the controlled displacement scenarios. Both the ultrasound transducer and the wall phantom, shown in figure 5.4, are immersed in water when the movements are applied. B-mode images are acquired using frame rate (FR) 10 frames/s and the RF echoes are acquired using 5-14 MHz linear-array ultrasound transducer. The probe is attached to a diagnostic ultrasound unit with special research capabilities (Sonix RP, Ultrasonix medical Corp., BC, Canada). A transmit frequency of $10 \mathrm{MHz}$ is used, and the radio frequency (RF) signals received from the FOV are digitized using sampling frequency of $40 \mathrm{MHz}$. The device was used in both the clinical and research modes to reconstruct images when different displacement scenarios are applied. These movement scenarios included sequences of pure radial, pure longitudinal and combined axial and longitudinal tiny displacements $(100-1000 \mu \mathrm{m})$ as $100 \mu \mathrm{m}$ pulses in order to mimic possible vascular wall movements in B-mode images. 


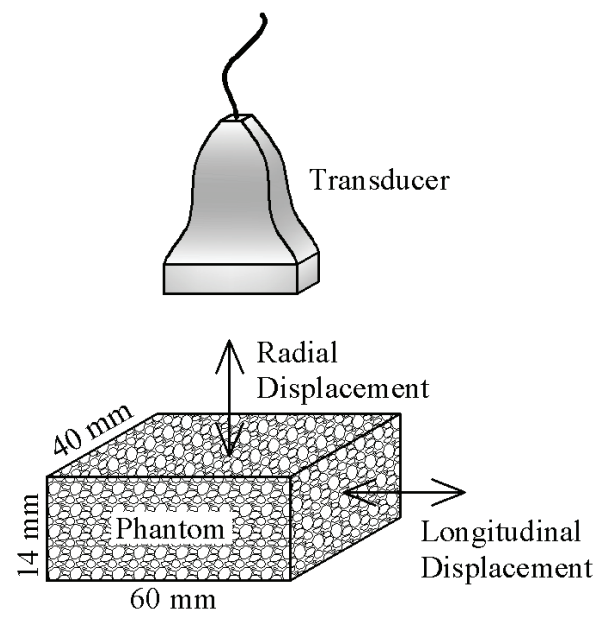

Figure 5.4 Simple schematic for the phantom experiment showing the radial and longitudinal directions. Pure radial, pure longitudinal and combined radial and longitudinal displacements are applied to the sponge phantom.

\subsubsection{In Vivo Flow Mediated Measurements}

For the in vivo measurements, ultrasound B-mode images of the right brachial artery of healthy subjects are collected. To assure versatility of the STM technique, two different ultrasound machines are used for image acquisition. The diagnostic ultrasound unit described above with its special research capabilities, and the 5-14 $\mathrm{MHz}$ linear-array ultrasound transducer is used. This machine is used in the clinical mode for vascular examination to provide B-mode processed images, and in the research mode to provide post-beamformed RF ultrasound signals. Post-beamformed RF signals are saved using 128 lines/frame received from the FOV and digitized using sampling frequency of $40 \mathrm{MHz}$. Also, a portable ultrasound machine using a probe specialized for vascular studies (Vivid-i, GE Healthcare, Milwaukee, WI) is used with its 4-13.3 MHz linear array ultrasound transducer. A transmit frequency range of 10$14 \mathrm{MHz}$ is used to ensure maximum resolution. Before initiating the study, participants were informed of all procedures and risks associated with the study; participants then signed an informed consent in accordance with the West Virginia University institutional review board for human subject experimentation. At rest, ultrasound frames of the brachial artery are collected continuously for about 20 seconds with different FR 6-26 frames/s. For FMD analysis, the brachial artery is 
occluded for 2 minutes using a standard adult pressure cuff with high pressure ( $>200$ $\mathrm{mmHg}$ ). Post-occlusion ultrasound images are then acquired after pressure release for $60 \mathrm{sec}$ using a temporal resolution $\mathrm{T}_{\mathrm{s}}(=1 / \mathrm{FR})$. Pre- and post-occlusion ultrasound data (signals and images) are then transferred to Matlab 7.1 (The MathWorks, Inc., Natick, MA, USA) for post processing and image reconstruction. After image preparation and initial processing procedures, i.e., filtration and contrast enhancement, all ROI within the images are segmented.

\subsection{Results and Discussion}

As a result from the experimental in vitro validation, figure 5.5 shows the displacement vectors when different displacement scenarios are applied. The arrows indicate the directions and the relative displacement amplitudes of measured values. Since the STM technique utilizes images acquired from the longitudinal section of the artery, the radial displacement can be approximated as the axial and similarly the longitudinal as the lateral. Figure 5.5(a) and (b) show the measured displacement arrows when $100 \mu \mathrm{m}$ longitudinal and radial displacements were applied, respectively, while figure 5.5(c) describes the measured displacements when both the $100 \mu \mathrm{m}$ longitudinal and radial displacements applied together. Results show good matching between the applied and measured displacement values. However, some regions have errors specially those on the boundary of the wall region due to very poor speckles. Different applied displacements $(100-1000 \mu \mathrm{m})$ were measured from different regions. The differences between the applied and measured displacements were evaluated, and the error was calculated in each scenario. An average displacement measurement error of $7 \%$ is estimated in both radial and longitudinal directions using various phantom experiments. There are different sources contributing to this error as will be discussed later in this section.

The STM technique has been tested in vivo using images acquired with two different ultrasound machines located at different research laboratories. Two sequences of B-mode images for the right brachial artery for the same subject were acquired, with the same sonographer acquiring both image sequences. Figure 5.6 (a) and (b) show B-mode images of the right brachial artery for a healthy 27 -year-old 
(a)
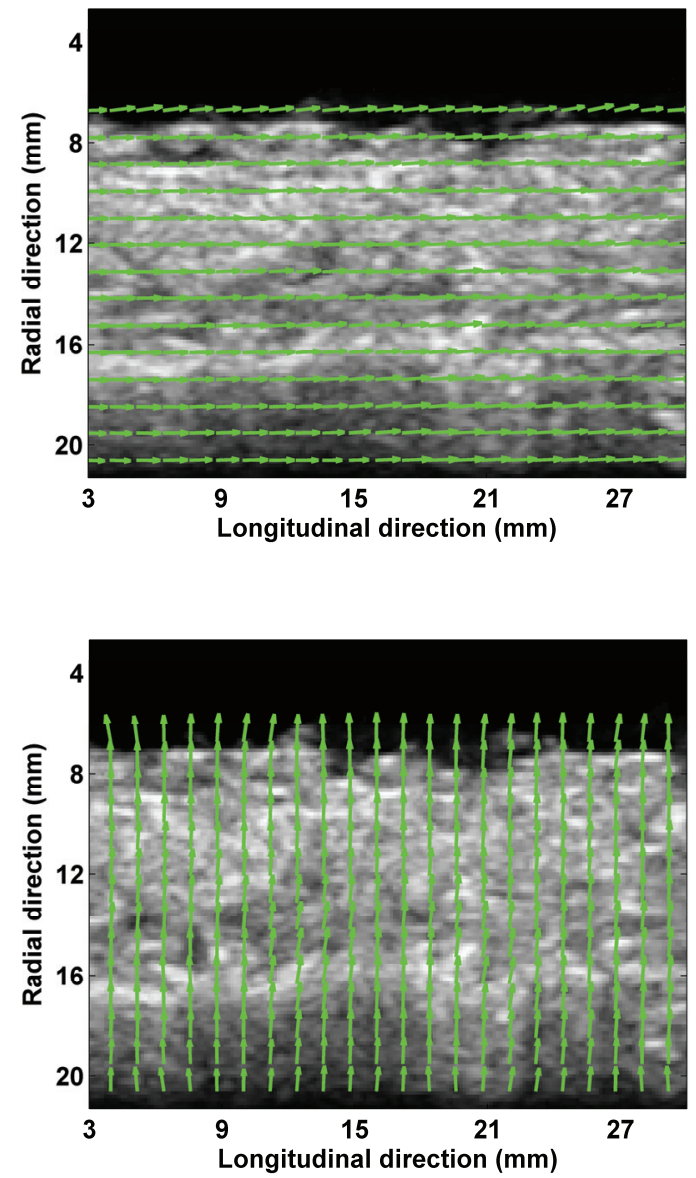

(b)

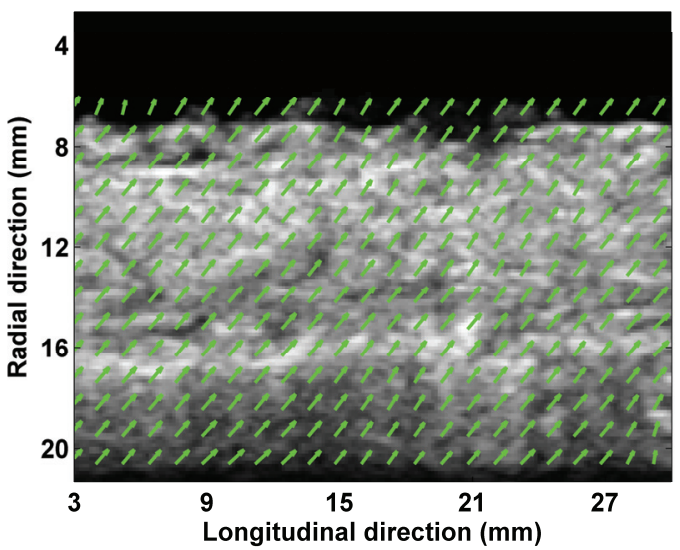

Figure 5.5 B-mode ultrasound images for the phantom when different displacements are applied. The arrows indicate the directions and the relative displacement amplitudes. (a) Pure longitudinal, (b) pure radial, and (c) both longitudinal and radial. 
male subject with the velocity vectors superimposed. The image shown in figure 5.6(a) and (b) were acquired using the GE and the Sonix RP systems, respectively. The arrows describe the relative velocity magnitude, or the frame to frame displacement at each kernel multiplied by the frame rate, and the direction of motion. Directions of arrows on both images may not be the same, since they are representing the movement between two different frame sequences within the cardiac cycle. It is the difficult to acquire two exact frame sequences in two different in vivo studies. However, the velocity ranges and averages for the two studies in both radial and

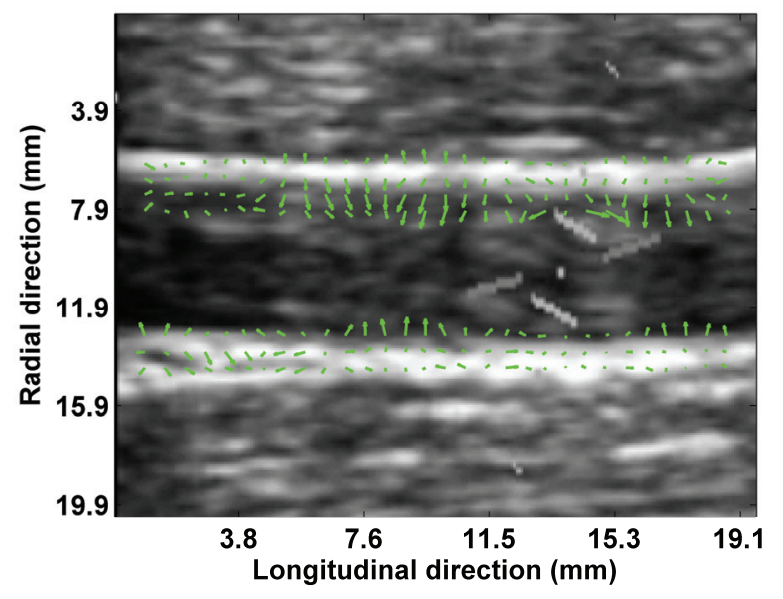

(a)

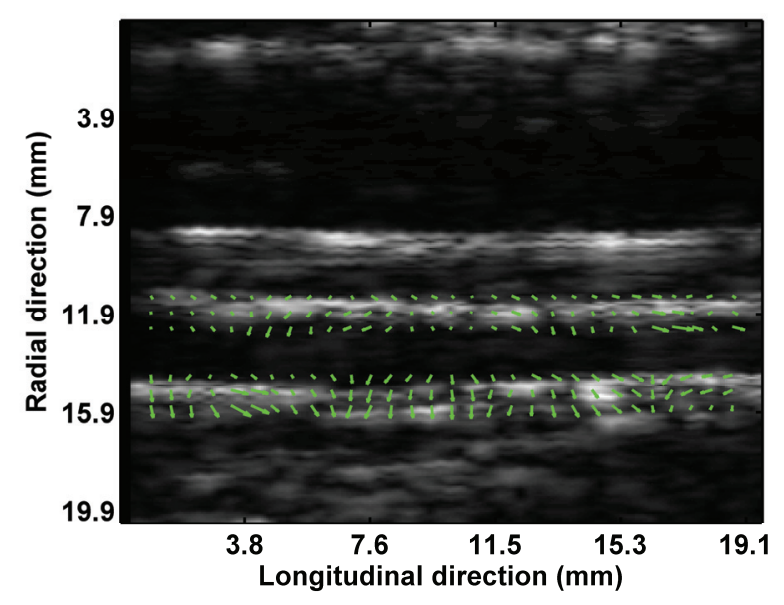

(b)

Figure 5.6 B-mode ultrasound images for the brachial artery of healthy subject (27year-old male). Superimposed arrows indicate the direction and amplitude of velocity components. (a) Image acquired using the GE system, and (b) image acquired with the Sonix RP system. 
longitudinal directions were matched under the same physiological conditions. The average radial velocity changes at two mid-locations on the near and far walls of healthy subject (26-year-old male) are extracted using B-mode images.

Figure 5.7(a) shows average velocity changes on both walls, where the near and far defines the relative locations from the ultrasound transducers. Radial displacements on both walls are calculated using temporal integration and the diameter change at this region was estimated as shown in figure 5.7(b). In spite of the small displacement components that the near wall exhibits, the value of diameter change $(\approx \pm 0.1 \mathrm{~mm})$ agrees with the manual measurements and the reported results by earlier studies [74-75]. This weak movement of the near wall may be a response due to the transducer pressure applied on the artery during acquisition. Such behavior was reported by Bambi et al. [71] as lower near wall displacement values than the far wall.

A small region ( $5 \mathrm{~mm}$ longitudinal $\times 2.5 \mathrm{~mm}$ radial) on the far wall has been used for calculating the vascular mechanical parameters to demonstrate our technique. Local wall strain tensors (radial, longitudinal, and shear strain) and the related strain rates are estimated during different conditions. Radial, longitudinal, and shear responses are shown in figure 5.8, where the strain rate and the corresponding strain curves during resting condition for a healthy subject (27-year-old male) are displayed. Based on different pilot studies for normal subjects, it is observed that the values of radial strain rate range from $0.2 \mathrm{~Hz}$ during diastole to $-0.1 \mathrm{~Hz}$ during systole, i.e. 0.3 Hz peak-to-peak change. By means of temporal integration, the average peak-to-peak change of radial strain is estimated to be in the range of 5\% per cardiac cycle under normal physiological conditions. These values are quite similar to the values reported in other studies for measuring brachial mechanics in vivo [97]. Peak-to-peak values for average changes of longitudinal and shear strain rates are also estimated to be 0.6 $\mathrm{Hz}$ and $0.4 \mathrm{~Hz}$, respectively. While the average peak-to-peak changes for longitudinal and shear strains are found to be respectively about $10 \%$ and $6 \%$ per cardiac cycle under normal physiological conditions. These average values of longitudinal and shear strains are quite larger when comparing with the radial strain, which could be due to wall shear stresses acting by the blood flow. The STM technique is also applied during different FMD studies. 

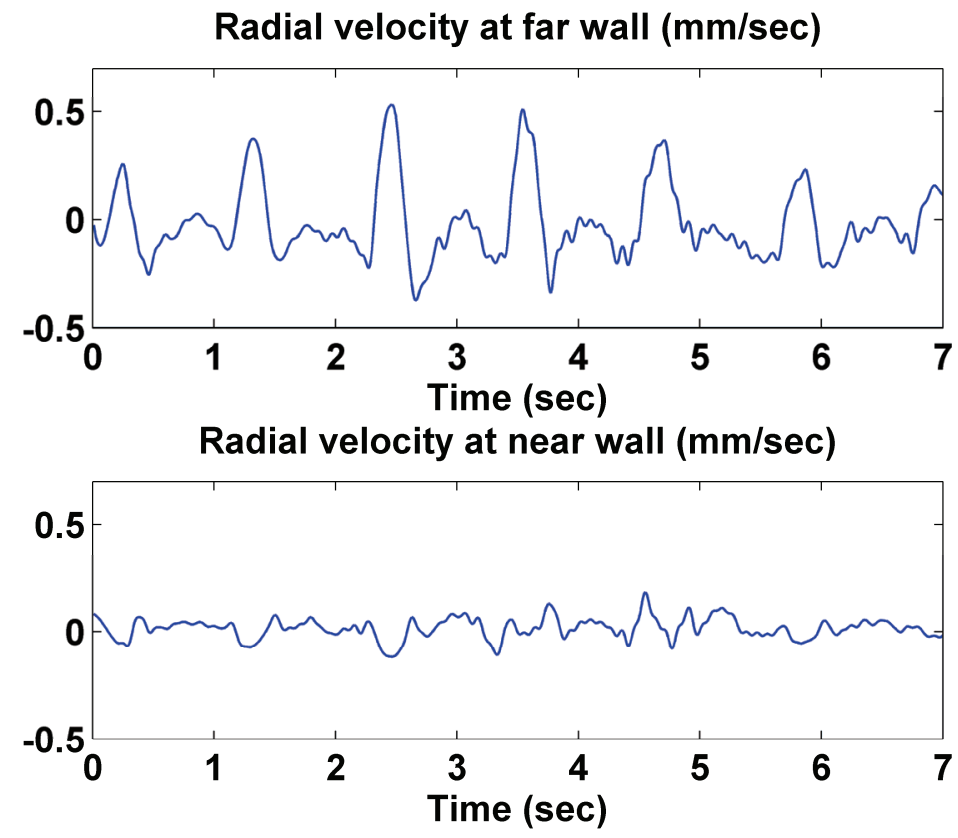

(a)

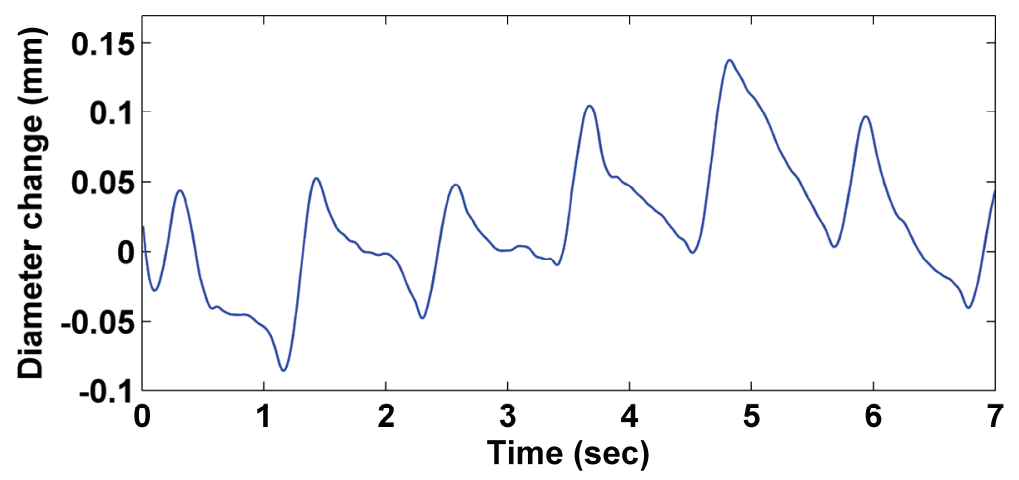

(b)

Figure 5.7 Average radial velocity and diameter changes measured within a region of interest within the brachial artery of a healthy subject (26-year-old male). (a) Velocity changes in both walls, and (b) diameter change.

In order to demonstrate the effect of the FMD response on changing wall mechanical parameters post-occlusion, a selected window of $22 \mathrm{sec}(25-47 \mathrm{sec})$ was chosen from the $60 \mathrm{sec}$ post-occlusion response. This window had most of the major changes in both diameter and wall parameters due to a 2 min occlusion for the healthy subject (26-year-old male). For this task, post-occlusion parameters change measured $25 \mathrm{sec}$ after pressure release for $22 \mathrm{sec}$ are compared to the pre-occlusion change. 

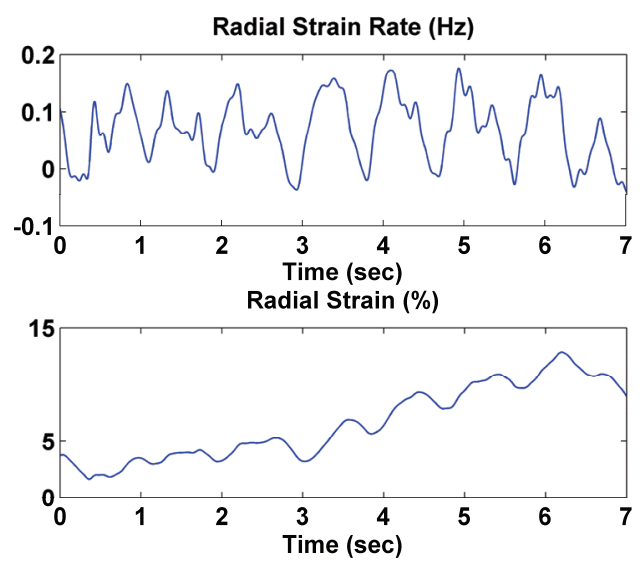

(a)
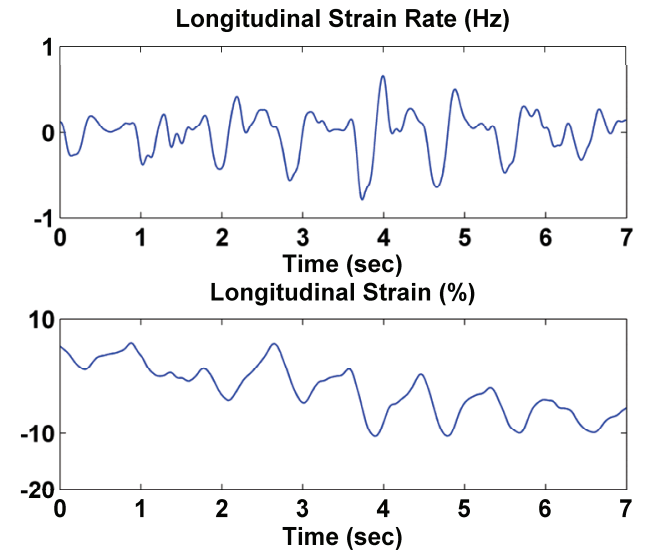

(b)
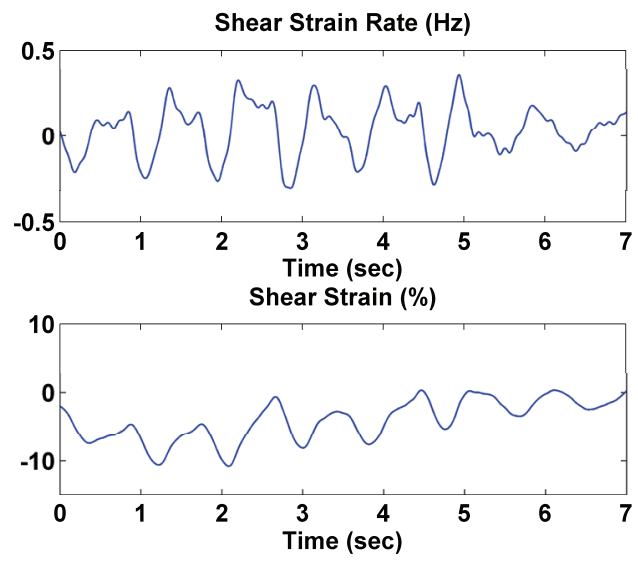

(c)

Figure 5.8 Average radial, longitudinal, and shear strain rates and strain components changes measured within a small region $(\approx 5 \mathrm{~mm} \times 2.5 \mathrm{~mm})$ on far wall of the brachial artery of healthy subject (27-year-old male). (a) Radial component, (b) longitudinal component, (c) and shear component. 
Figure 5.9(a) shows the diameter change where the average peak-to-peak value during pre-occlusion period is about $0.1 \mathrm{~mm}$. After pressure release, twice this average is observed in figure 5.9(b). Moreover, in some locations the diameter changes have reached $0.3 \mathrm{~mm}$ per cycle. Superimposed arrows refer to places where

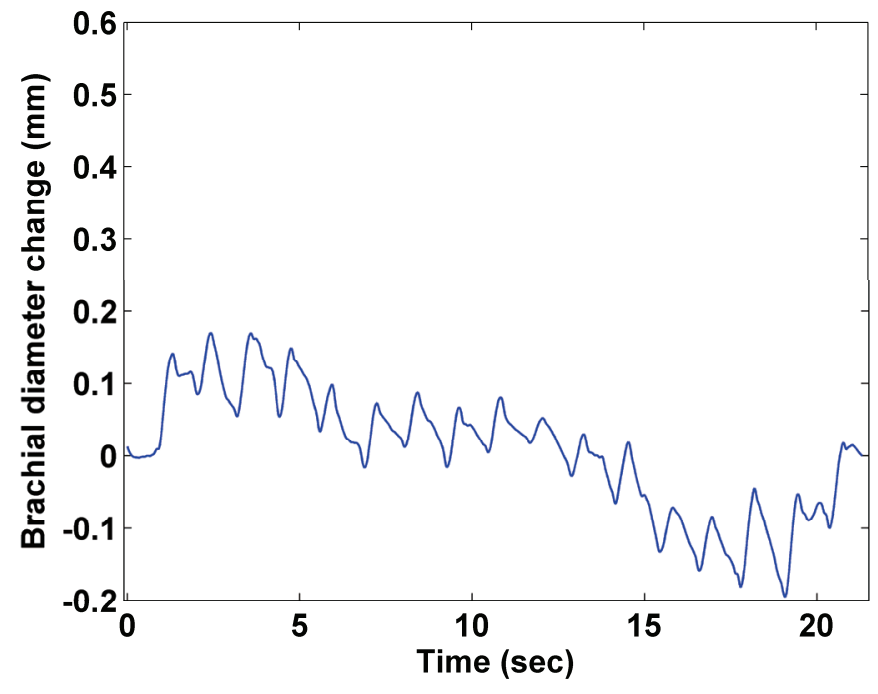

(a)

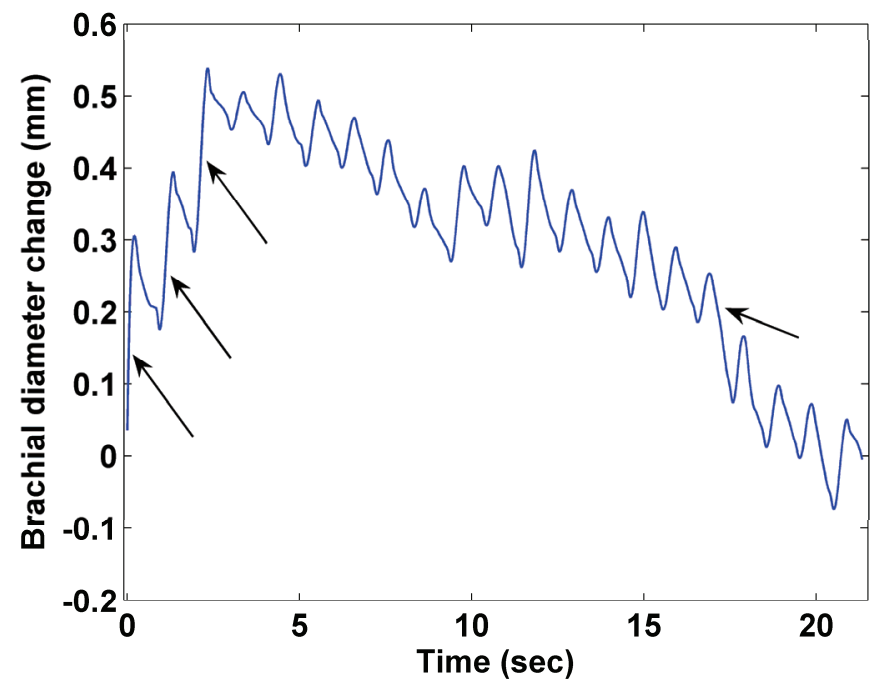

(b)

Figure 5.9 Average diameter change pre- and post-occlusion (about $20 \mathrm{sec}$ after pressure release) for healthy subject (26-year-old male). (a) pre-occlusion, and (b) post-occlusion. 
maximum change in diameter per cycle is observed. Changes on the values of radial strain and strain rates are estimated during the same analysis. Arrows pointing in figure 5.10 show that the radial strain rate changes per cardiac cycle have reached
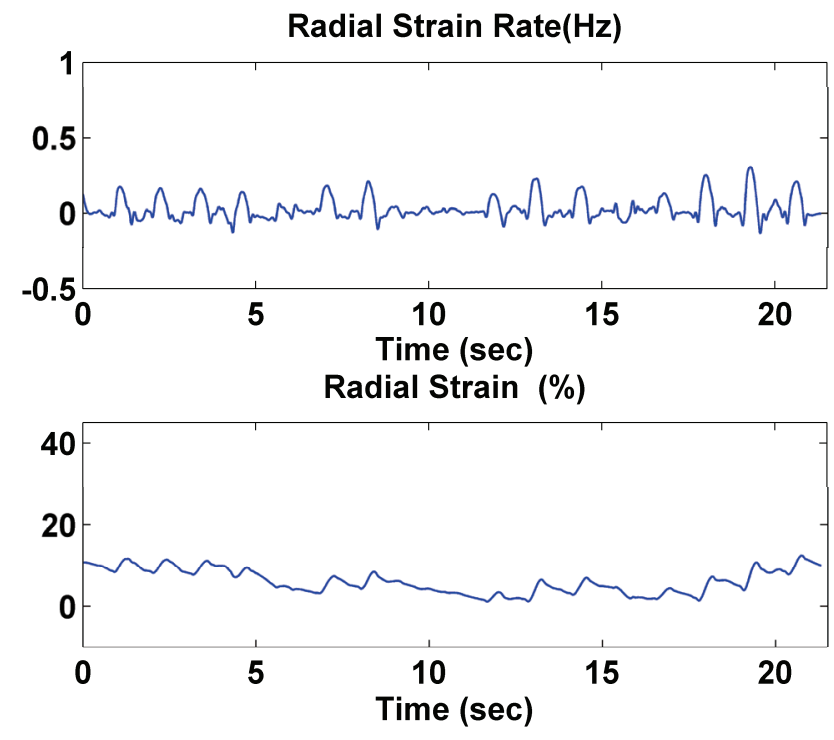

(a)

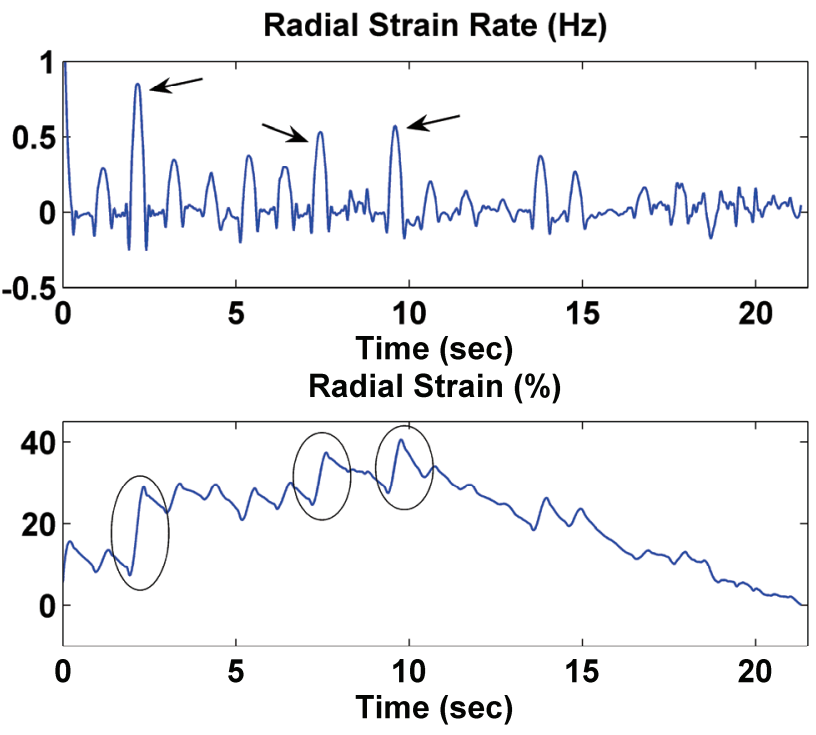

(b)

Figure 5.10 Average radial strain rate and strain change pre- and post-occlusion. Values are measured within a small region $(\approx 5 \mathrm{~mm} \times 2.5 \mathrm{~mm})$ on far wall of the brachial artery of healthy subject (26-year-old male). (a) Pre-occlusion, and (b) postocclusion. 
more than $0.5 \mathrm{~Hz}$, which is about twice the nominal values. Same behavior is observed when comparing radial strain values during pre- and post-occlusion. The circles in figure $5.10(\mathrm{~b})$ clarify some locations where changes of strain values per cardiac cycle exceed $10 \%$ and $15 \%$, i.e., twice or more the nominal value.

Figure 5.11 compares the pre- and post-occlusion behaviors of the longitudinal

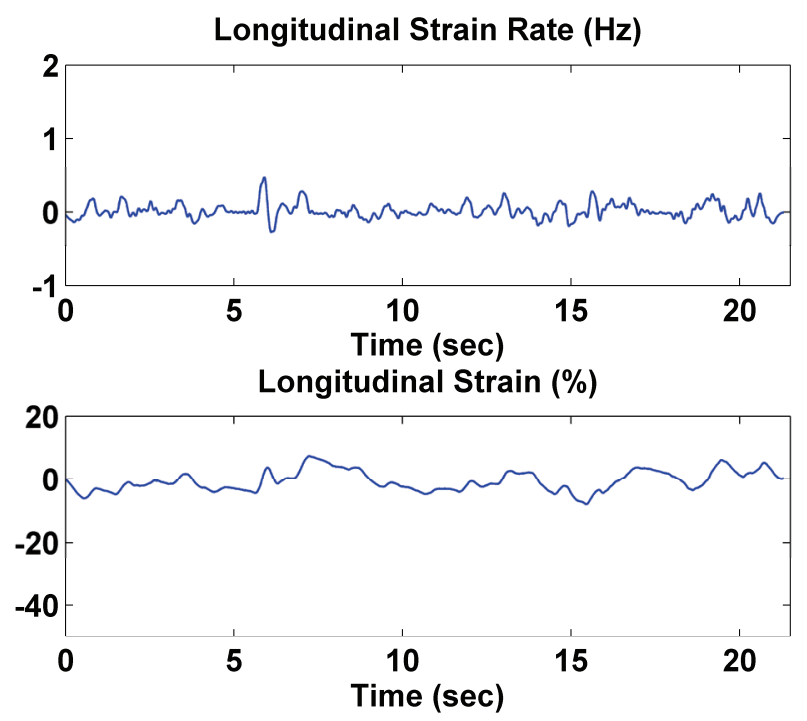

(a)
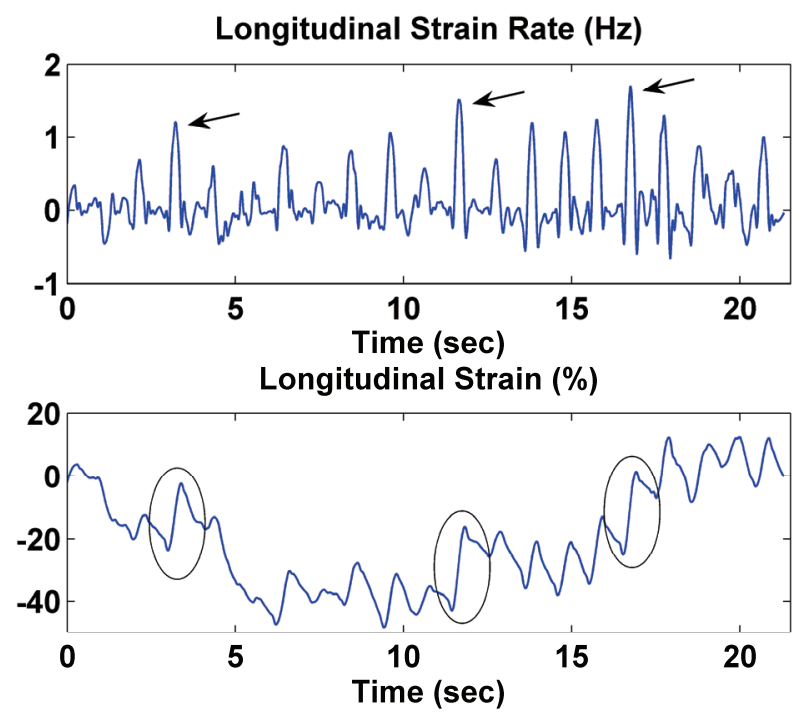

(b)

Figure 5.11 Average longitudinal strain rate and strain change pre- and postocclusion. Values are measured within a small region $(\approx 5 \mathrm{~mm} \times 2.5 \mathrm{~mm})$ on far wall of the brachial artery of healthy subject (26-year-old male). (a) Pre-occlusion, and (b) post-occlusion. 
strain and strain rate changes. The behavior of the longitudinal strain and strain rates is quite similar to the radial ones, where the longitudinal strain rate values exceed 1.2 $\mathrm{Hz}$, and the longitudinal strain changes exceed $15 \%$ in some locations, i.e. twice or more than the nominal values. The arrows and circles superimposed on figure 5.11 refer to the locations where remarkable changes exist for longitudinal strain rate and strain, respectively. Changes in shear strain and strain rates during pre- and postocclusion are shown in figure 5.12. It is observed that changes of shear strain and shear strain rate values have reached more than $15 \%$ and $0.8 \mathrm{~Hz}$, respectively per cardiac cycle. This response of the shear strain and strain rate after pressure release is also similar to that observed for radial and longitudinal components.

Based on the physiology behind the FMD analysis, this stimulus provokes the endothelium to release nitric oxide with subsequent vasodilatations that can be imaged and quantified as an index of vasomotor function [104]. So, the origin of any stimulus is initiated from the vascular wall, which can be measured directly using STM technique. In this work, it is observed that not only diameter change that may exhibits major changes in the measurements during pre- and post-occlusion, but also there are other parameters such as strain and strain rates that may be used in the FMD analysis. Also, it is observed that comparing the average peak-to-peak values per cardiac cycle could be another method for FMD assessment. This shall reduce the processing time and save efforts since long term recording would not be needed.

The STM technique was applied in vivo on 5 subjects to compare the postocclusion response of the diameter change and other measured parameters. In order to test the correlation between the diameter change and wall strains responses, the crosscorrelation function was evaluated using Matlab 'crosscorr' function with a 95\% confidence interval. Table 5.1 shows the amplitude of the correlation coefficient $|\mathrm{r}|$ estimated at zero lags (no signal shift is applied) between the diameter change and wall strains (radial, longitudinal, and shear) for 5 subjects. Generally, it was observed that most of the post-occlusion responses of diameter change and wall strains exhibit good correlation $(|r|>0.5)$. Correlations of $|r|=0.66 \pm 0.17, \quad|r|=0.71 \pm 0.05$, and $|r|=0.70 \pm 0.19(\mathrm{n}=5, \mathrm{P}<0.05)$ were found between the diameter change response and the radial, longitudinal, and shear strain post-occlusion responses, respectively. Few responses show quite low correlation $(|\mathrm{r}|<0.5)$ such as the diameter change response versus the radial strain response of the 72-year-old male patient. However for the 
same subject, the correlations between the diameter change and both shear and longitudinal responses were fairly good $(|r| \geq 0.7)$. This behavior may be due to a time
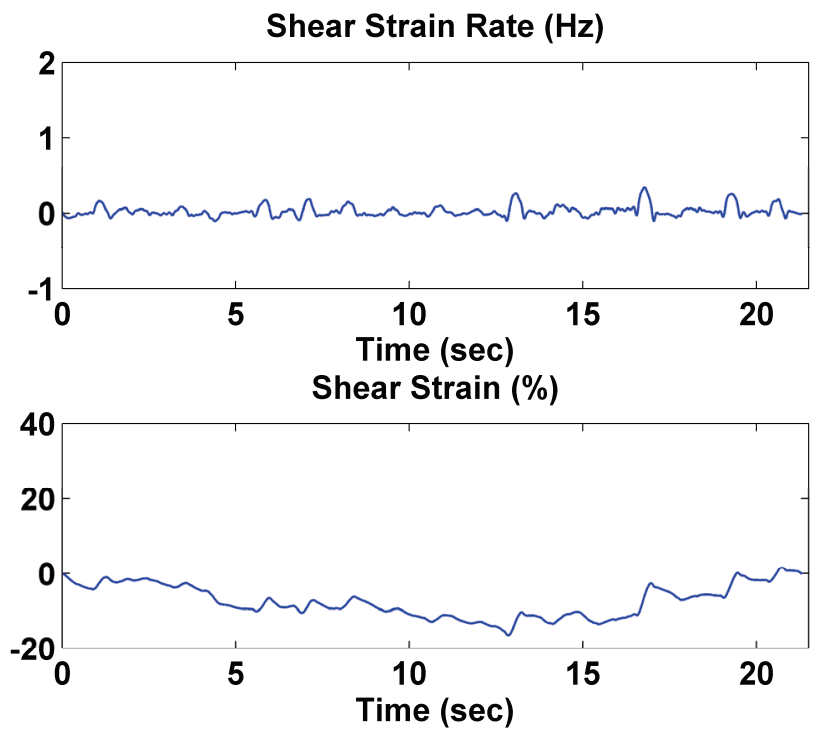

(a)
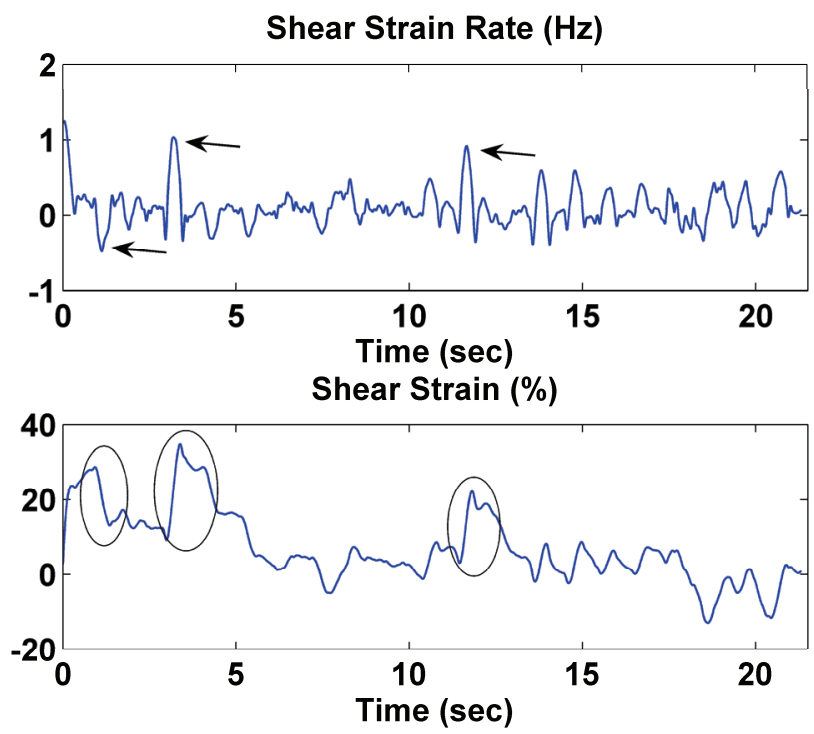

(b)

Figure 5.12 Average shear strain rate and strain change pre- and post-occlusion. Values are measured within a small region $(\approx 5 \mathrm{~mm} \times 2.5 \mathrm{~mm})$ on far wall of the brachial artery of healthy subject (26-year-old male). (a) Pre-occlusion, and (b) postocclusion. 
Table 5.1 Correlation $(|\mathrm{r}|)$ between the post-occlusion response of diameter change and local wall strains (radial, longitudinal, and shear) for 5 subjects. The amplitude of correlation coefficient $|\mathrm{r}|$ is calculated using 95\% confidence interval $(\mathrm{P}<0.05)$.

\begin{tabular}{c|c|c|c|c|c|c|c}
\hline $\begin{array}{c}\text { Subject } \\
\begin{array}{l}\text { Parameter } \\
\text { Correlation }(|\mathrm{r}|)\end{array}\end{array}$ & $\begin{array}{c}\text { 72-year- } \\
\text { old male } \\
\text { (Patient) }\end{array}$ & $\begin{array}{c}\text { 62-year- } \\
\text { old male } \\
\text { (Patient) }\end{array}$ & $\begin{array}{c}\text { 63 year- } \\
\text { old male } \\
\text { (Control) }\end{array}$ & $\begin{array}{c}\text { 26 year- } \\
\text { old male } \\
\text { (Control) }\end{array}$ & $\begin{array}{c}\text { 27 year- } \\
\text { old male } \\
\text { (Control) }\end{array}$ & Mean & $\begin{array}{c}\text { Standard } \\
\text { Deviation }\end{array}$ \\
\hline Radial Strain $|\mathrm{r}|$ & 0.47 & 0.59 & 0.87 & 0.81 & 0.56 & 0.66 & \pm 0.17 \\
\hline Long. Strain $|\mathrm{r}|$ & 0.7 & 0.74 & 0.74 & 0.62 & 0.72 & 0.71 & \pm 0.05 \\
\hline Shear Strain $|\mathrm{r}|$ & 0.75 & 0.45 & 0.88 & 0.55 & 0.86 & 0.70 & \pm 0.19 \\
\hline
\end{tabular}

lag between the radial strain and diameter change responses, or due to measurement errors as will be discussed later. On the other hand, some responses show significant correlation $(|r|>0.85)$ such as the diameter change and shear responses of the 27 -yearold male healthy subject. In the above correlation analysis, only wall strains have been used to represent the parameters since it is evaluated directly from the strain rates. Also, the sign of the correlation coefficient has been ignored since the direction of correlation is not our focus in this study.

Other parameters could be estimated from measured values, figure 5.13 describes a comparison between the frequency spectrum for the radial wall motion pre- and post-occlusion. It is observed that the frequency spectrum, shown in figure 5.13 (a), has two main features; one peak corresponds to the fundamental frequency at 0.8905 $\mathrm{Hz}$, and another peak to the second harmonic at $1.871 \mathrm{~Hz}$, which is exactly as twice as the fundamental frequency. After pressure release, the value of the fundamental frequency shown in figure 5.13 (b) is still the same, but the second harmonic has been shifted slightly to be $1.93 \mathrm{~Hz}$. This response could be normal since the fundamental frequency depends mainly on the heart rate/pulse, which is not affected by pressure release. Moreover, the slight shifting is the second harmonic component could be due to the rapid response of the artery after release. Indeed, the large radial deformation of the arterial wall during post-occlusion will cause a strong nonlinear arterial wall mechanics due to tissue nonlinearity [105-107]. This behavior may suggest that the 
second harmonic (i.e., displacement component and frequency-shift) to be another parameters that may be used for FMD analyses.

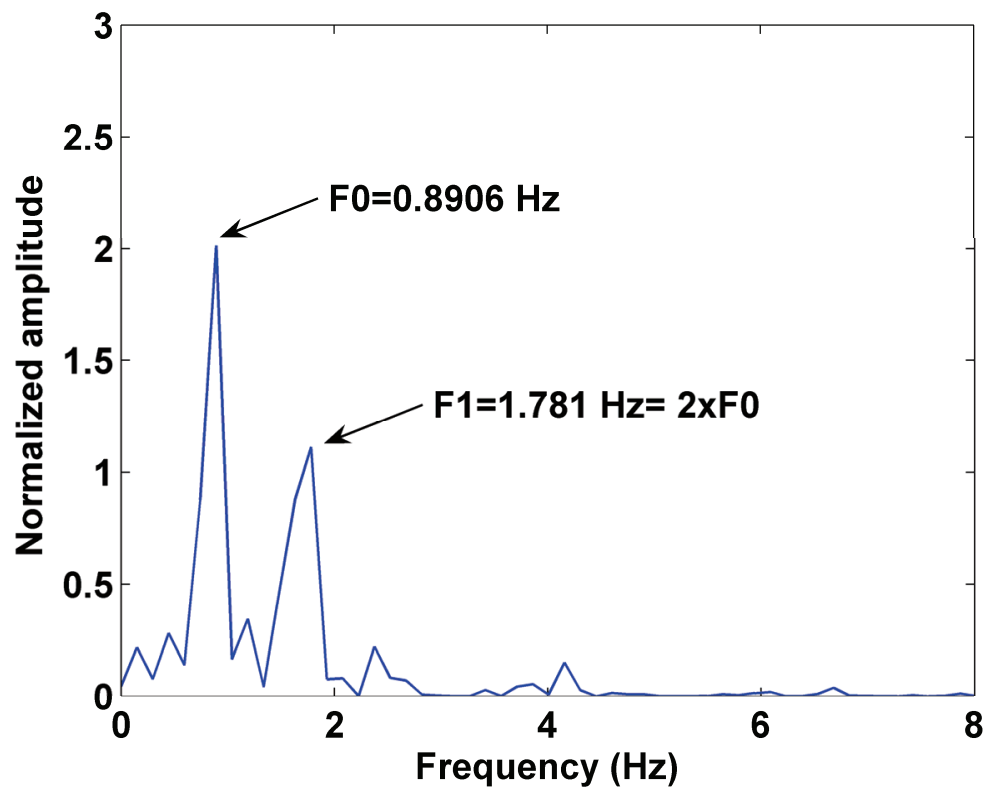

(a)

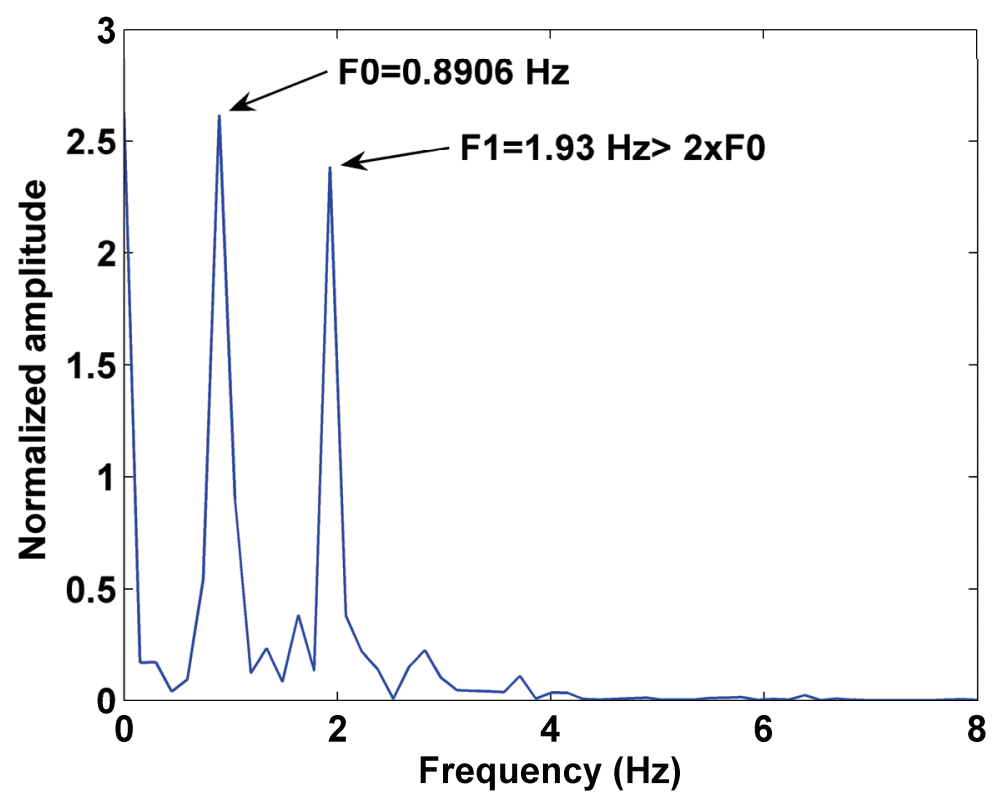

(b)

Figure 5.13 Frequency spectrum of the radial component of the far wall motion of healthy subject (26-year-old male). (a) Pre-occlusion, and (b) post-occlusion. 
Although the STM algorithm demonstrates an efficient tool for measuring arterial strain components, there are various factors that may affect its accuracy. Human motion error from both operator and patient could be among these factors. However it could be reduced via manufacturing special holders for the patient arm or the ultrasound transducer as in [75]. One more source of motion error that is impossible to eliminate is the environmental vibrations. The lack of speckles could be also a source of error which may affect the accuracy of the algorithm. This effect has been shown in the in vitro results, figure 5.5, where in some regions near the boundaries there are some errors due to poor speckles. This effect could be reduced through segmenting speckle-reach regions and avoid selecting the boundaries. Also, speckle decorrelation along the transducer beam direction could affect the accuracy of tracking the speckles. However, this effect could be minor when using the electronically beam-formed transducer arrays which are able to produce fully developed speckle over a much greater range by maintaining focus over an infinite depth of focus [108]. All transducers used in this work are electronically focused. Moreover, these arrays are the most commonly used in current ultrasound machines for cardiovascular applications.

The STM technique could be applied on any region on both boundaries which are full of speckles. However, we suggest using the far wall in calculating the arterial parameters during the FMD studies, because of two main reasons. The first reason is to avoid the effect of ultrasound transducer pressure applied by the sonographer on patient's arm during image acquisition. This pressure may affect parameter values calculated from the near wall, and is difficult to standardize. Such effect was reported by Bambi et al. [71] as lower near wall displacement values. The other reason is that the far wall usually has a well defined structure that can be easily segmented than the near wall. This feature is due to the irregular echoes received from the adventitia extend into the media and the intima on the near wall [109]. Moreover, Gemignani et al. [75] recommended using the far wall as it could be located more easily and more accurately than the near wall. However, this characteristic is dependent of the ultrasound machine used, and could be overcome by the using proper image processing procedures that enhances the image quality and improves the edges, and/or by using the right acquisition parameters such as the electronic focus and gain. 
In this work, we have used a 2 min occlusion period to produce the FMD response rather than using the commonly used 4-5 min period. We believe that at the short end of the spectrum (i.e. $2 \mathrm{~min}$ ), dilator/hyperemic responses tend to be more a function of shear and less a function of the others (withdrawal of adrenergic tone/sympatholysis, myogenic dilation with the loss of pressure and metabolic dilation). The peak flow change reaches a maximum after 1.5 min of cuff occlusion, but the maximum vessel dilation may not occur until 4.5 min occlusion [110]. In this study, the vessel has been stimulated to produce the FMD response; however the maximum vessel dilatation may not be reached. Several groups have demonstrated that the 2 min occlusion is able to produce the FMD response, particularly due to wall stresses [110-112].

In this work, manual segmentation is used; but in the future, automatic segmentation with image processing algorithms may be used to avoid this problem. This STM technique does not require ultrahigh frame rate, since in this paper the frame rate does not exceed 26 frames/sec. The time integration of some parameters, such as the strain rate, can result in a drifting if the frame rate is not high enough. However using physical boundary condition that the strain value at the end of the cardiac cycle should be zero can automatically compensate this effect [101]. Drifting compensation has not been applied in this study, since the main focus is to explain and test the algorithm. 


\section{CHAPTER 6}

\section{CONCLUSION AND FUTURE WORK}

\subsection{Concluding Remarks}

In the first part of this thesis (chapter 3), we presented two novel techniques based on ultrasound that can be used to assess mechanical changes and detect small defects within human jawbones. First, we investigated the possibility of using ultrasonic guided waves to assess jawbone. Experimental studies were performed in vitro using healthy and defected mandibles to obtain the dispersion behavior of guided waves in the frequency range of $0.7-1.2 \mathrm{MHz}$. The experimental set up for the guided wave experiments was also described. Wavelet-based technique was used to calculate guided wave arrival time using the Gabor wavelet. Experimental results were compared with the theoretical results of a previous study performed in our laboratory for guided wave propagation within jawbone. Results show variation in the theoretically and experimentally calculated group velocities according to the mandibular condition. Both the theoretical and experimental studies indicate that guided waves can be used in assessing the mechanical properties and detecting small bony defects of human jawbone. Moreover, this study shows that lower frequency guided wave transducers will be useful for clinical diagnosis using extensional and flexural wave modes. In the second method, a digital high frequency ultrasound imaging system for jawbone scanning was described. The system is able to reconstruct 3D surface images for the jawbone outer surface with high resolution down to $50 \mu \mathrm{m}$. This system was tested using in vitro experiments using human dentate and non-dentate mandibles. Ultrasound signal and image processing techniques are used to detect accurately opaque jawbone boundaries and defects in human mandibles. Results showed that the system is capable in detecting minute bony defects in the jawbone and provide a promising non-invasive technique to aid in the diagnosis of periodontal disease. Comparisons between ultrasound and X-ray images indicate superiority of the ultrasound imaging technique in identifying defects in the lateral, axial, and elevation directions quantitatively. The limitations of 
conventional 2D radiographs in diagnosing $3 \mathrm{D}$ periodontal defects can be circumvent by our High frequency 3D echodentographic imaging technique.

The characterization technique, described in the middle part of this thesis (chapter $4)$, is based on imaging small parts with microscopic resolution $(<50 \mu \mathrm{m})$. A high frequency precise single element ultrasound imaging system for small animals was developed and tested in vitro using wire phantoms and mouse hearts and blood vessels. This custom designed system relies on a high speed precise positioning system $(1 \mu \mathrm{m})$. The system is equipped with different high frequency ultrasound transducers of 30,60 , and $100 \mathrm{MHz}$ in center frequency. This high frequency ultrasound system is capable of providing spatial resolution down to $10.3 \mu \mathrm{m}$. Adaptive weighted SAFT technique is applied on received signals for focusing. Weighting windows were used for apodization, and various window types were tested. Moreover, advanced image processing techniques are applied for image enhancement and resolution improvement. Several phantom experiments were performed using single and five pin wire phantoms to assure the system focusing ability and focusing homogeneity at different depths. Henceforth, the system is capable of achieving uniform spatial resolution at different depths and can overcome the problem of resolution degradation. The system achieved an experimental axial spatial resolution of $18 \mu \mathrm{m}$ using the $25 \mu \mathrm{m}$ wire. Several in vitro studies for different models of isolated mouse hearts were performed to demonstrate the system efficacy. Experimental results for the mouse hearts shows good matching with the heart anatomy images, and provides high quality B-mode images. Currently the system can reconstruct high resolution accurate B-mode images for in vitro biological tissues and will provide a significance help for cardiovascular researcher using animal models, especially when the high frame rate is provided.

In the last part of this thesis (chapter 5), a strain tensor measuring (STM) technique that provides noninvasive measurements of the brachial wall mechanical changes is presented. This algorithm is simple, fast, and can measure different parameters directly from the vascular wall. Moreover, no extra hardware is required which make the technique flexible and universal. Both in vitro phantom experiments and in vivo clinical studies were performed to evaluate the algorithm efficiency and repeatability. Also, the technique was applied during post-occlusion reactive hyperemia and the flow-mediated vasodilation (FMD) analysis. An average error of 
$7 \%$ was reported using different phantom studies. During pre- and post-occlusion, in vivo results show drastic changes in both shear and longitudinal mechanical parameters other than the diameter change, which has been in common use for FMD analysis. Moreover, good correlations $(|\mathrm{r}|>0.5, \mathrm{P}<0.05)$ were found between the postocclusion response of diameter change and the responses of local wall strains based on five in vivo studies. This suggests that monitoring the local strain and strain rates of the brachial artery is likely to be useful in measuring the changes in arterial tone due to flow mediated endothelial stimulation. Therefore, these parameters could replace or augment the measurement of arterial diameter in FMD studies. This algorithm could be integrated easily with any commercial ultrasound machine which shall benefit the cardiovascular research field.

\subsection{Future Work}

We believe that each of the abovementioned techniques, presented in this thesis, has some challenging problems and limitations that could be overcome in future work. The 3D ultrasound echodentographic imaging system for human mandibles requires further in vitro validations. Fresh defected cadaver mandibles with human tissue will be the ideal target to image in order to mimic the in vivo environment. Then, slight modification in the system design or the signal/image processing procedures could be required in order to move toward the in vivo testing stage. This may include changing the transducer placement, scanning procedures, and jawbone edge detection algorithm. Upon finalizing the system prototype, 100-200 in vivo experiments could be used for the final validation before moving to the FDA approval and toward having a commercial system. Moreover, automatic classification for the gingiva would be a beneficial addition to the system, besides imaging.

Regarding the high-frequency ultrasound imaging system for small animals, incorporating the high frame rate has become a must after validating the high resolution. This can be achieved via using a programming language such as visual $\mathrm{C} / \mathrm{C}++$ to control and synchronize the system, and to process and display the signals/images. Also, synchronizing the data acquisition with the electrocardiogram (ECG) signal of the small animal could help in making the system in vivo. In addition, further in vitro and in vivo validations are required using different mouse 
models. Moreover, developing automatic characterization techniques based on both envelop and final images will improve the system, and facilitate the assessment process. These characterization techniques can utilize the properties of the spectral and/or Wavelet data of reconstructed images.

Regarding the STM technique, developed for cardiovascular tissue characterization, additional testing using a larger population of human subjects will be beneficial to validate the correlation between the diameter change and other parameters. This will facilitate proposing the new STM-FMD method that could be used for non-invasive diagnosis of cardiovascular diseases. In addition, adding the automatic segmentation property to the STM technique and developing a friendly graphical user interface (GUI) will facilitate the use of such technique. The GUI for the STM could be implemented and tested using visual $\mathrm{C} / \mathrm{C}++$ on an ultrasound machine with research capabilities. Finally, as we noticed a complex movement of multiple waves traveling via the vascular wall when applying the STM technique, we believe that further analysis for the waveform travelling along the wall would be beneficial. 


\section{REFERENCES}

[1] D. A. Christensen, Ultrasonic Bioinstrumentation, New York: Wiley, 1988.

[2] T. L. Szabo, Diagnostic Ultrasound Imaging Inside Out, Academic Press, 2004.

[3] C. Passmann, and H. Ermert, "In vivo imaging of the skin in the $100 \mathrm{MHz}$ region using the synthetic aperture concept," in Proceedings IEEE Ultrasonics Symposium, 1995, pp. 1287-1290.

[4] B. S. Garra, E. I. Cespedes, J. Ophir, S. R. Spratt, R. A. Zuurbier, C. M. Magnant, and M. F. Pennanen, "Elastography of breast lesions: initial clinical results," Radiology, vol. 202(1), pp. 79-86, 1997.

[5] M. F. Insana and D. G. Brown. Ultrasonic Scattering in Biological Tissues, CRC Press, Boca Raton, 1993.

[6] J. M. Thijssen, "Ultrasonic tissue characterization," Acoustical Imaging, vol. 25, pp. $12-25,2000$.

[7] K. Horner, H. Devlin, C. W. Alsop, I. M. Hodgkinson, and J. E. Adams, "Mandibular bone mineral density as a predictor of skeletal osteoporosis," The British Journal of Radiology, vol. 69, pp. 1019-1025, 1996.

[8] J. Lynch, M. Hinders, and G. McCombs, "Clinical comparison of an ultrasonographic periodontal probe to manual and controlled-force probing," Measurement, vol. 39, no. 5, 429-439, 2006.

[9] G. V. Black, A work on Special Dental Pathology: Devoted to the Diseases and Treatment of the Investing Tissues of the Teeth and the Dental Pulp. MedicoDental Publ. Co., Chicago, IL, 1924.

[10] T. Levy, and H. Huggins, "Routine dental extractions routinely produce cavitations," Journal of Advancement in Medicine, vol. 9, no. 4, pp. 235-249, 1996.

[11] M. Huysmans, and J. Thijssenb, "Ultrasonic measurement of enamel thickness: a tool for monitoring dental erosion?” Journal of Dentistry, vol. 28, pp. 187-191, 2000. 
[12] S. Toda, T. Fujita, H. Arakawa, and K. Toda, "An ultrasonic nondestructive technique for evaluating layer thickness in human teeth," Sensors and Actuators A: Physical, vol. 125, no. 1, pp. 1-9, 2005.

[13] M. Culjat, R. Singh, E. Brown, R. Neurgaonkar, D. Yoon, and S. White, "Ultrasound crack detection in a simulated human tooth," Dentomaxillofacial Radiol, vol. 34, no. 2, pp. 80-85, 2005.

[14] J. Lynch, and M. Hinders, "Ultrasonic device for measuring periodontal attachment levels," Review of Scientific Instruments, vol. 73, No. 7, pp. 2686$2693,2002$.

[15] F. Tsiolis, I. Needleman, and G. Griffiths, "Periodontal ultrasonography," Journal Clinical Periodontology, vol. 30, pp. 849-854, 2003.

[16] E. Cotti, G. Campisi, V. Garau, and G. Puddu, "A new technique for the study of periapical bone lesions: ultrasound real time imaging," International Endodontic Journal, vol. 35, pp. 148-152, 2002.

[17] H. Uchida, K. Kobayashi, and M. Nagao, "Measurement in vivo of masticatory mucosal thickness with $20 \mathrm{MHz}$ B-Mode ultrasonic diagnostic equipment," Journal of Dental Research, vol. 68, no. 2, pp. 95-100, 1989.

[18] J. Bouquot, W. Martin, G. Wrobleski, "Computer based thru-transmission sonography (CTS) imaging of ischemic osteonecrosis of the jaws: A preliminary investigation of 6 cadaver jaws and 15 pain patient," Oral Surgery, Oral Medicine, Oral Pathology, Oral Radiology, and Endodontics, vol. 92, pp. 550, 2001.

[19] J. Imbeau, "Introduction to through-transmission alveolar ultrasonography (TAU) in dental medicine," Cranio, the Journal of Craniomandibular Practice, vol. 23, no. 2, pp. 100-112, 2005.

[20] A. J. Niklasson, S. K. Datta, and M. L. Dunn, "On ultrasonic guided waves in a thin anisotropic layer lying between two isotropic layers," Journal of the Acoustical Society of America, vol. 108, no. 5, pp. 2005-2011, 2000.

[21] A. Mal, "Elastic waves from localized sources in composite laminates," International Journal of Solids and Structures, vol. 39, pp. 5481-5494, 2002. 
[22] Z. Su, L. Ye, Y. Lu, "Guided Lamb waves for identification of damage in composite structures: A review," Journal of Sound and Vibration, vol. 295, pp. 753-780, 2006.

[23] R. Y. Vasudeva, and P. G. Rao, "Influence of voids in interface zones on lambmode spectra in fiber-reinforced composite laminates," Journal of Applied Physics, vol. 71, no. 2, pp. 612-619, 1992.

[24] B. C. Lee, and W. J. Staszewski, "Modelling of Lamb waves for damage detection in metallic structures: Part II. Wave interactions with damage," Smart Materials and Structures, vol. 12, no. 5, pp. 815-824, 2003.

[25] E. Pan, J. Rogers, S. K. Datta, and A. H. Shah, "Mode selection of guided waves for ultrasonic inspection of gas pipelines with thick coating," Mechanics of Materials, vol. 31, no. 3, pp. 165-174, 1999.

[26] O. M. Mukdadi, and S. K. Datta, "Transient ultrasonic guided waves in layered plates with rectangular cross section," Journal of Applied Physics, vol. 93, no. 11, pp. 9360-9370, 2003.

[27] P. Cawley, M. J. Lowe, D. N. Alleyne, B. Pavlakovic, and P. Wilcox, "Practical long range guided wave testing: applications to pipes and rail," Materials Evaluation, vol. 61, no. 1, pp. 66-74, 2003.

[28] T. Hayashi, W-J Song, and J. L. Rose, "Guided dispersion curves for a bar with an arbitrary cross-section, a rod and rail example," Ultrasonics, vol. 41, pp. 175183, 2003.

[29] C. Njeh, C. Boivin, and C. Langton, "The role of ultrasound in the assessment of osteoporosis: A review," Osteoporosis International, vol. 7, pp. 7-22, 1997.

[30] E. Bossy, M. Talmant, F. Peyrin, L. Akrout, P. Cloetens, and P. Laugier, “An in vitro study of the ultrasonic axial transmission technique at the radius: $1-\mathrm{MHz}$ velocity measurements are sensitive to both mineralization and intracortical porosity,” Journal of Bone Mineral Research, vol. 19, no. 9, pp. 1548-1556, 2004.

[31] E. Bossy, M. Talmant, M. Defontaine, F. Patat, and P. Laugier, "Bidirectional axial transmission can improve accuracy and precision of ultrasonic velocity measurement in cortical bone: A validation on test materials," IEEE Transactions 
on Ultrasonics Ferroelectrics and Frequency Control, vol. 51, no. 1, pp. 71-79, 2004.

[32] K. Raum, I. Leguerney, F. Chandelier, E. Bossy, M. Talmant, A. Saied, F. Peyrin, and P. Laugier, "Bone microstructure and elastic tissue properties are reflected in QUS axial transmission measurements," Ultrasound in Medicine and Biology, vol. 31, no. 9, pp. 1225-1235, 2005.

[33] P. Moilanen, P Nicholson, T. Karkkainen, Q. Wang, J. Timonen, and S. Cheng, "Assessment of the tibia using ultrasonic guided waves in pubertal girls," Osteoporosis International, vol. 14, pp. 1020-1027, 2003.

[34] P. Nicholson, P. Moilanen, T. Karkkainen, J. Timonen, and S. Cheng, "Guided ultrasonic waves in long bones: modeling, experiment and in vivo application," Physiological Measurement, vol. 23, pp. 755-768, 2002.

[35] K. Lee, and S. Yoon, "Feasibility of bone assessment with leaky Lamb waves in bone phantoms and a bovine tibia," Journal of the Acoustical Society of America, vol. 115, no. 6, pp. 3210-3217, 2004.

[36] F. Lefebvre, Y. Deblock, P. Campistron, D. Ahite, and J. Fabre, "Development of a new ultrasonic technique for bone and biomaterials in vitro characterization,", Journal of Biomedical Materials Research, vol. 63, no. 4, pp. 441-446, 2002.

[37] A. Daugherty, "Mouse models of atherosclerosis," American Journal of the Medical Sciences, vol. 323, pp. 3-10, 2002.

[38] J. L. Breslow, "Transgenic mouse models of lipoprotein metabolism and atherosclerosis," Proceedings of the National Academy of Sciences of the United States of America, vol. 90, pp. 8314-8318, 1993.

[39] M. E. Johansson, U. Hagg, J. Wikstrom, A. Wickman, G. Bergstrom, and L. M. Gan, "Haemodynamically significant plaque formation and regional endothelial dysfunction in cholesterol-fed ApoE(-/-) mice," Clinical Science, vol. 108, pp. $531-538,2005$.

[40] S. H. Zhang, R. L. Reddick, J. A. Piedrahita, and N. Maeda, "Spontaneous hypercholesterolemia and arterial lesions in mice lacking apolipoprotein-E," Science, vol. 258, pp. 468-471, 1992. 
[41] G. H. Lee, R. Proenca, J. M. Montez, K. M. Carroll, J. G. Darvishzadeh, J. I. Lee, and J. M. Friedman, "Abnormal splicing of the leptin receptor in diabetic mice," Nature, vol. 379, pp. 632-635, 1996.

[42] P. K. Mazumder, B. T. O'Neill, M. W. Roberts, J. Buchanan, U. J. Yun, R. C. Cooksey, S. Boudina, and E. D. Abel, "Impaired cardiac efficiency and increased fatty acid oxidation in insulin-resistant ob/ob mouse hearts," Diabetes, vol. 53, pp. 2366-2374, 2004.

[43] J. C. Russell and S. D. Proctor, "Small animal models of cardiovascular disease: tools for the study of the roles of metabolic syndrome, dyslipidemia, and atherosclerosis," Cardiovascular Pathology, vol. 15, pp. 318-330, 2006.

[44] F. Jiang, G. T. Jones, A. J. Husband, and G. J. Dusting, "Cardiovascular protective effects of synthetic isoflavone derivatives in apolipoprotein E-deficient mice," Journal of Vascular Research, vol. 40, pp. 276-284, 2003.

[45] S. A. Schreyer, D. L. Wilson, and R. C. LeBoeuf, "C57BL/6 mice fed high fat diets as models for diabetes-accelerated atherosclerosis," Atherosclerosis, vol. 136, pp. 17-24, 1998.

[46] G. Christensen, Y. B. Wang, and K. R. Chien, "Physiological assessment of complex cardiac phenotypes in genetically engineered mice," American Journal of Physiology-Heart and Circulatory Physiology, vol. 41, pp. H2513-H2524, 1997.

[47] D. Baumgart, A. Schmermund, G. Goerge, M. Haude, J. B. Ge, M. Adamzik, C. Sehnert, K. Altmaier, D. Groenemeyer, R. Seibel, and R. Erbel, "Comparison of electron beam computed tomography with intracoronary ultrasound and coronary angiography for detection of coronary atherosclerosis," Journal of the American College of Cardiology, vol. 30, pp. 57-64, 1997.

[48] H. Sakuma, N. Kawada, K. Takeda, and C. B. Higgins, "MR measurement of coronary blood flow," Journal of Magnetic Resonance Imaging, vol. 10, pp. 728$733,1999$.

[49] G. Y. Chang, F. Cao, M. Krishnan, M. Huang, Z. J. Li, X. Y. Xie, A. Y. Sheikh, G. Hoyt, R. C. Robbins, T. Hsial, M. D. Schneider, and J. C. Wu, "Positron 
emission tomography imaging of conditional gene activation in the heart," Journal of Molecular and Cellular Cardiology, vol. 43, pp. 18-26, 2007.

[50] S. N. Roper, M. D. Moores, G. V. Gelikonov, F. I. Feldchtein, N. M. Beach, M. A. King, V. M. Gelikonov, A. M. Sergeev, and D. H. Reitze, "In vivo detection of experimentally induced cortical dysgenesis in the adult rat neocortex using optical coherence tomography," Journal of Neuroscience Methods, vol. 80, pp. 91-98, 1998.

[51] O. Aristizabal, D. A. Christopher, F. S. Foster, and D. H. Turnbull, “40-MHz echocardiography scanner for cardiovascular assessment of mouse embryos," Ultrasound in Medicine and Biology, vol. 24, pp. 1407-1417, 1998.

[52] K. Kramer, S. Vanacker, H. P. Voss, J. A. Grimbergen, W. J. F. Vandervijgh, and A. Bast, "Use of telemetry to record electrocardiogram and heart-rate in freely moving mice," Journal of Pharmacological and Toxicological Methods, vol. 30, pp. 209-215, 1993.

[53] T. Makela, P. Clarysse, O. Sipila, N. Pauna, Q. C. Pham, T. Katila, and I. E. Magnin, "A review of cardiac image registration methods," IEEE Transactions on Medical Imaging, vol. 21, pp. 1011-1021, 2002.

[54] D. H. Turnbull, T. S. Bloomfield, H. S. Baldwin, F. S. Foster, and A. L. Joyner, "Ultrasound backscatter microscope analysis of early mouse embryonic braindevelopment," Proceedings of the National Academy of Sciences of the United States of America, vol. 92, pp. 2239-2243, 1995.

[55] F. S. Foster, M. Y. Zhang, Y. Q. Zhou, G. Liu, J. Mehi, E. Cherin, K. A. Harasiewicz, B. G. Starkoski, L. Zan, D. A. Knapik, and S. L. Adamson, "A new ultrasound instrument for in vivo microimaging of mice," Ultrasound in Medicine and Biology, vol. 28, pp. 1165-1172, 2002.

[56] F. S. Foster, C. J. Pavlin, K. A. Harasiewicz, D. A. Christopher, and D. H. Turnbull, "Advances in ultrasound biomicroscopy," Ultrasound in Medicine and Biology, vol. 26, pp. 1-27, 2000.

[57] S. Srinivasan, H. S. Baldwin, O. Aristizabal, L. Kwee, M. Labow, M. Artman, and D. H. Turnbull, "Noninvasive, in utero imaging of mouse embryonic heart 
development with 40-MHz echocardiography," Circulation, vol. 98, pp. 912-918, 1998.

[58] E. Cherin, R. Williams, A. Needles, G. W. Liu, C. White, A. S. Brown, Y. Q. Zhou, and F. S. Foster, "Ultrahigh frame rate retrospective ultrasound microimaging and blood flow visualization in mice in vivo," Ultrasound in Medicine and Biology, vol. 32, pp. 683-691, 2006.

[59] M. Lukacs, J. H. Yin, G. F. Pang, R. C. Garcia, E. Cherin, R. Williams, J. Mehi, and F. S. Foster, "Performance and characterization of new micromachined highfrequency linear arrays," IEEE Transactions on Ultrasonics Ferroelectrics and Frequency Control, vol. 53, pp. 1719-1729, 2006.

[60] J. A. Brown, E. S. Foster, A. Needles, E. Cherin, and G. R. Lockwood, "Fabrication and performance of a 40-MHz linear array based on a 1-3 composite with geometric elevation focusing," IEEE Transactions on Ultrasonics Ferroelectrics and Frequency Control, vol. 54, pp. 1888-1894, 2007.

[61] C. H. Hu, X. C. Xu, J. M. Cannata, J. T. Yen, and K. K. Shung, "Development of a real-time, high-frequency ultrasound digital beamformer for high-frequency linear array transducers," IEEE Transactions on Ultrasonics Ferroelectrics and Frequency Control, vol. 53, pp. 317-323, 2006.

[62] W. Rosamond, K. Flegal, G. Friday, K. Furie, A. Go, K. Greenlund, N. Haase, M. Ho, V. Howard, B. Kissela, S. Kittner, D. Lloyd-Jones, M. McDermott, J. Meigs, C. Moy, G. Nichol, C. J. O'Donnell, V. Roger, J. Rumsfeld, P. Sorlie, J. Steinberger, T. Thom, S. Wasserthiel-Smoller, Y. Hong, for the American Heart Association Statistics Comm, (2007). Heart Disease and Stroke Statistics--2007 Update: A Report from the American Heart Association Statistics Committee and Stroke Statistics Subcommittee. Circulation 115: e69-e171.

[63] R. Ross, "Atherosclerosis - An inflammatory disease," The New England journal of medicine, vol. 340, pp. 115-126, 1999

[64] T. Wada, and T. Fukumoto, "Biomechanical diagnosis of atherosclerosis by ultrasound," Methods of Information in Medicine Journal, vol. 39, no. 3, pp. 246284, 2000 . 
[65] T. Reichlin, A. Widl, M. Durrenberger, A. U. Daniels, U. Aebi, P. R. Hunziker, and M. Stolz, "Investigating native coronary artery endothelium in situ and in cell culture by scanning force microscopy," Journal of Structural Biology, vol. 152, pp. 52-63, 2005.

[66] K. Hayashi, H. Handa, A. Nagasawa, A. Okumura, and K. Moritake, "Stiffness and elastic behavior of human intracranial and extracranial arteries," Journal of Biomechanics, vol. 13, pp. 175-184, 1980.

[67] K. V. Ramnarine, T. Hartshorne, Y. Sensier, M. Naylor, J. Walker, A. R. Naylor, R. B. Panerai, and D. H. Evans, "Tissue Doppler imaging of carotid plaque wall motion: a pilot study," Cardiovascular Ultrasound, vol. 1, pp.1:17, 2003.

[68] P. J. Brands, A. P. Hoeks, L. Hofstra, R. S. Reneman, “A noninvasive method to estimate wall shear rate using ultrasound," Ultrasound in Medicine and Biology, vol. 21, pp. 171-185, 1995.

[69] A. P. Hoeks, T. G. Arts, P. J. Brands, R. S. Reneman, "Processing scheme for velocity estimation using ultrasound RF cross correlation techniques," European Journal of Ultrasound, vol. 1, pp. 171-182, 1994.

[70] P. J. Brands, A. P. Hoeks, J. Willigers, C. Willekes, and R. S. Reneman, “An integrated system for the non-invasive assessment of vessel wall and hemodynamic properties of large arteries by means of ultrasound," European Journal of Ultrasound, vol. 9, pp. 257-266, 1999.

[71] G. Bambi, T. Morganti, S. Ricci, E. Boni, F. Guidi, C. Palombo and P. Tortoli, "A novel ultrasound instrument for investigation of arterial mechanics," Ultrasonics, vol. 42, pp. 731-737, 2004.

[72] P. Tortoli, T. Morganti, G. Bambi, C. Palombo, and K. V. Ramnarine, "Noninvasive simultaneous assessment of wall shear rate and wall distension in carotid arteries," Ultrasound in Medicine and Biology, vol. 32, no. 11, pp. 1661$1670,2006$. 
[73] L.I. Sinoway, C. Hendrickson, W.R. Davidson, S. Prophet, and R. Zelis, "Characteristics of flow-mediated brachial artery vasodilation in human subjects," Circulation Research, vol. 64, pp. 32-42., 1989

[74] L. Fan, P. Santago, H. Jiang, and D. M. Herrington, "Ultrasound Measurement of Brachial Flow-Mediated Vasodilator Response," IEEE Transactions on Medical Imaging, vol. 19, pp. 621-631. , 2000.

[75] V. Gemignani, V. Faita, L. Ghiadoni, E. Poggianti, and M. Demi, 2007, “A System for Real-Time Measurement of the Brachial Artery Diameter in B-Mode Ultrasound Images," IEEE Transactions on Medical Imaging, vol. 26, pp. 393404.

[76] T. Kaneko, H. Hasegawa and H. Kanai, "Ultrasonic Measurement of Change in Elasticity due to Endothelium Dependent Relaxation Response by Accurate Detection of Artery-Wall Boundary," Japanese Journal of Applied Physics, vol. 46, pp. 4881-4888, 2007.

[77] A. M. Mahmoud, D. Cortes, A. Abaza, H. Ammar, M. Hazey, P. Ngan, R. Crout, and O. M. Mukdadi, "Non-Invasive Assessment of Human Jawbone Using Ultrasonic Guided Waves," IEEE Transactions on Ultrasonics, Ferroelectrics, and Frequency Control, vol. 55, pp. 1316-1327, 2008.

[78] A. M. Mahmoud, D. Cortes, A. Abaza, H. Ammar, M. Hazey, P. Ngan, R. Crout, and O. M. Mukdadi, “Assessment of Human Jawbone Using Ultrasonic Guided Wave: In Vitro Study," in Proceedings of IEEE International Ultrasonics Symposium, 2007, pp. 2183-2186.

[79] M. A. Hamstad, An Illustrated Overview of the Use and Value of a Wavelet Transformation to Acoustic Emission Technology, University of Denver, Denver, CO, USA, 2003.

[80] L. Wang, and F. G. Yuan, "Group velocity and characteristic wave curves of Lamb waves in composites: Modeling and experiments," Composites Science and Technology, vol. 67, pp. 1370-1384, 2007. 
[81] H. Jeong, and Y. S. Jang, "Fracture source location in thin plates using the wavelet transform of dispersive waves," IEEE Transactions on Ultrasonics, Ferroelectrics, and Frequency Control, vol. 47, pp. 612-619, 2000.

[82] P. C. Dechow, G. A. Nail, C. L. Schwartz-Dabney, and R. B. Ashman, "Elastic properties of human supraorbital and mandibular bone," American Journal of Physical Anthropology, vol. 3, pp. 91-116, 1993.

[83] M. Zybutz, D. Rapoport, L. Laurell, and G. Persson, "Comparisons of clinical and radiographic measurements of inter-proximal vertical defects before and 1 year after surgical treatments," Journal of Clinical Periodontology, vol. 3, pp. 179-186, 2000.

[84] M. Goodson, A.D. Haffajee, and S.S. Socransky, "The relationship between attachment loss and alveolar bone loss", Journal of Clinical Periodontology, vol. 11, no. 5, pp. 348-359, 1984.

[85] W. Xu, R. Siffert, and J. Kaufman, "Application of wavelet analysis to ultrasonic characterization of bone", proceedings of the 28th Asilomar Conference on Signals, Systems, and Computers, 1994, vol.2, pp. 1090 - 1094.

[86] M. Karaman, P. C. Li, and M. O'Donnell, "Synthetic-aperture imaging for small-scale systems," IEEE Transactions on Ultrasonics Ferroelectrics and Frequency Control, vol. 42, pp. 429-442, 1995.

[87] C. H. Frazier and W. D. O'Brien, "Synthetic aperture techniques with a virtual source element," IEEE Transactions on Ultrasonics Ferroelectrics and Frequency Control, vol. 45, pp. 196-207, 1998.

[88] M. L. Li, W. J. Guan, and P. C. Li, "Improved synthetic aperture focusing technique with applications in high-frequency ultrasound imaging," IEEE Transactions on Ultrasonics Ferroelectrics and Frequency Control, vol. 51, pp. 63-70, 2004.

[89] J. G. Proakis and D. K. Manolakis, Digital Signal Processing: Principles, Algorithms, and Applications, New York: MacMillan, 1992. 
[90] P. Kovesi, "Phase Preserving Denoising of Images," Proceedings of The Australian Pattern Recognition Society Conference: DICTA'99. Perth WA. 1999, pp. 212-217.

[91] L. Sun, W. D. Richard, J. M. Cannata, C. C. Feng, J. A. Johnson, J. T. Yen, and K. K. Shung, "A high-frame rate high-frequency ultrasonic system for cardiac imaging in mice," IEEE Transactions on Ultrasonics Ferroelectrics And Frequency Control, vol. 54, pp. 1648-1655, 2007.

[92] P. A. Jansson, Deconvolution of Images and Spectra, Academic Press, San Diego, CA, 1997.

[93] P. Perona and J. Malik, "Scale-space and edge-detection using anisotropic diffusion," IEEE Transactions on Pattern Analysis and Machine Intelligence, vol. 12, pp. 629-639, 1990.

[94] L. N. Bohs, and G. E. Trahey, "A novel method for angle independent ultrasonic-imaging of blood-flow and tissue motion," IEEE Transactions On Biomedical Engineering, vol. 38, pp. 280-286, 1991.

[95] W. F. Walker, B. H. Friemel, L. N. Bohs, and G. E. Trahey, "Real-time imaging of tissue vibration using a two-dimensional speckle tracking system," Proceedings of the IEEE Ultrasonics Symposium, 1993, pp. 873-876.

[96] M. O'Donnell, A. R. Skovoroda, B. M. Shapo, and S. Y. Emelianov, "Internal displacement and strain imaging using ultrasonic speckle tracking," IEEE Transactions On Ultrasonics Ferroelectrics And Frequency Control, vol. 41, pp. 314-25, 1994.

[97] K. Kim, W. F. Weitzel, J. M. Rubin, H. Xie , X. C. Chen, and M. O’Donnell, "Vascular intramural strain imaging using arterial pressure equalization," Ultrasound in Medicine and Biology, vol. 30, pp. 761-71, 2004.

[98] L. N. Bohs, B. H. Friemel, B. A. McDermott, and G. E. Trahey, "Real-Time System For Angle-Independent Us Of Blood-Flow In 2 Dimensions - Initial Results,” Radiology, vol. 186, pp. 259-261, 1993.

[99] B. H. Friemel, L. N. Bohs, and G. E. Trahey, "Relative performance of twodimensional speckle- tracking techniques: normalized correlation, non- 
normalized correlation and sum-absolute difference," Proceedings of the IEEE Ultrasonics Symposium, 1995. vol. 2, pp. 1481-1484.

[100] N. Mori, and K-A Chang, Introduction to MPIV -PIV toolbox in MATLAB, 0.965 ed, 2006.

[101] J. D'hooge, A. Heimdal, F. Jamal, T. Kukulski, B. Bijnens, F. Rademakers, L. Hatle, P. Suetens, and G. R. Sutherland, "Regional Strain and Strain Rate Measurements by Cardiac Ultrasound: Principles, Implementation and Limitations," European Journal of Echocardiography, vol. 1, 2000.

[102] S. Srinivasan , T. Krouskop, and J. Ophir, "A quantitative comparison of modulus images obtained using nanoindentation with strain elastograms," Ultrasound in Medicine and Biology, vol. 30, pp. 899-918, 2004

[103] G. E. Trahey, S. W. Smith, and O. T. Vonramm, "Speckle pattern correlation with Lateral aperture translation - experimental results and implications for spatial compounding," IEEE Transactions on Ultrasonics Ferroelectrics and Frequency Control, vol. 33, pp. 257-264, 1986.

[104] M. C. Corretti, T. J. Anderson, E. J. Benjamin, D. Celermajer, F. Charbonneau, M. A. Creager, J. Deanfield, H. Drexler, M. Gerhard-Herman, D. Herrington, P. Vallance, J. Vita, and R. Vogel, "Guidelines for the ultrasound assessment of endothelial-dependent flow-mediated vasodilation of the brachial artery - a report of the international brachial artery reactivity task force," Journal of the American College of Cardiology, vol. 39, pp. 257-265, 2002.

[105] J. E. Bischoff, E. M. Arruda, and K. Grosh, "A microstructurally based orthotropic hyperelastic constitutive law," Journal of Applied Mechanics, vol. 69, pp. 570-579, 2002.

[106] J. D. Humphrey, "Continuum biomechanics of soft biological tissues," Proceedings of the Royal Society. A-Mathematical Physical and Engineering Sciences, vol. 459, pp. 3-46, 2003.

[107] R. P. Vito, and S. A. Dixon, "Blood vessel constitutive models-1995-2002," Annual Review of Biomedical Engineering, vol. 5, pp. 413-439, 2003. 
[108] B. M. Shapo, J. R. Crowe, A. R. Skovoroda, M. J. Eberle, N. A. Cohn, and M. O'Donnell, "Displacement and strain imaging of coronary arteries with intraluminal ultrasound," IEEE Transactions on Ultrasonics Ferroelectrics and Frequency Control, vol. 43, pp. 234-246, 1996.

[109] R. S. Reneman, J. M. Meinders, and A. P. Hoeks, "Non-invasive ultrasound in arterial wall dynamics in humans: What have we learned and what remains to be solved," European Heart Journal, vol. 26, pp. 960-966, 2005.

[110] P. Leeson, S. Thorne, A. Donald, M. Mullen, P. Clarkson, and J. Deanfield, "Non-invasive measurement of endothelial function: effect on brachial artery dilatation of graded endothelial dependent and independent stimuli," Heart, vol. 78, pp. 22-27, 1997.

[111] S. Laurent, P. Lacolley, P. Brunel, B. Laloux, B. Pannier, and M. Safar, "Flow-dependent vasodilation of brachial-artery in essential-hypertension," American Journal of Physiology-Heart and Circulatory, vol. 258, pp. H1004H10011, 1990.

[112] J. Padilla, B. D. Johnson, S. C. Newcomer, D. P. Wilhite, T. D. Mickleborough, A. D. Fly, K. J. Mather, and J. P. Wallace, "Normalization of flow-mediated dilation to shear stress area under the curve eliminates the impact of variable hyperemic stimulus," Cardiovascular Ultrasound, vol. 6, pp. 44, 2008.

[113] A. M. Mahmoud, S. Jamal Mustafa, and O. M. Mukdadi, "Ultrahigh Resolution Quantitative Ultrasound Imaging System for Small Animal Imaging," Proceedings of the ASME International Mechanical Engineering Congress and Exposition, Boston, MA, 2008.

[114] A. M. Mahmoud, D. H. Cortes, S. Jamal Mustafa, and O. M. Mukdadi, "Ultrasound Imaging System to Assess Mouse Hearts and Blood Vessels," Proceedings of the Summer Bioengineering Conference, Marco Island, Florida 2008.

[115] A. M. Mahmoud, P. Stapleton, J. Frisbee, O. M. Mukdadi "Noninvasive Measurement of Brachial Wall Mechanics During Flow-Mediated Vasodilation Using 2D Ultrasound Strain Tensor Imaging," Proceedings of the Summer Bioengineering Conference, Marco Island, Florida 2008. 